\title{
On an equation being a fractional differential equation with respect to time and a pseudo-differential equation with respect to space related to Levy-type processes.
}

\author{
$\mathrm{Hu}, \mathrm{Ke}$
}

How to cite:

$\mathrm{Hu}, \mathrm{Ke}(2012)$ On an equation being a fractional differential equation with respect to time and a pseudo-differential equation with respect to space related to Levy-type processes.. thesis, Swansea University.

http://cronfa.swan.ac.uk/Record/cronfa43021

Use policy:

This item is brought to you by Swansea University. Any person downloading material is agreeing to abide by the terms of the repository licence: copies of full text items may be used or reproduced in any format or medium, without prior permission for personal research or study, educational or non-commercial purposes only. The copyright for any work remains with the original author unless otherwise specified. The full-text must not be sold in any format or medium without the formal permission of the copyright holder. Permission for multiple reproductions should be obtained from the original author.

Authors are personally responsible for adhering to copyright and publisher restrictions when uploading content to the repository.

Please link to the metadata record in the Swansea University repository, Cronfa (link given in the citation reference above.) 


\title{
On an Equation being a Fractional Differential Equation with respect to \\ Time and a Pseudo-Differential Equation with respect to Space related to Lévy-type Processes
}

\author{
$\mathrm{Ke} \mathrm{Hu}$ \\ 465765
}

Submitted to the Swansea University in fulfillment of the requirements for the Degree of Doctor of philosophy

Swansea University

2012 
ProQuest Number: 10821411

All rights reserved

INFORMATION TO ALL USERS

The quality of this reproduction is dependent upon the quality of the copy submitted.

In the unlikely event that the author did not send a complete manuscript and there are missing pages, these will be noted. Also, if material had to be removed, a note will indicate the deletion.

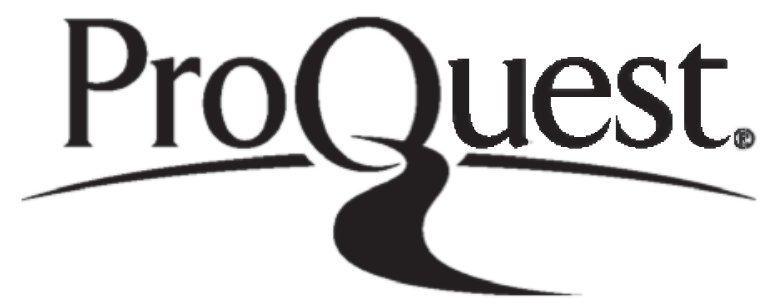

ProQuest 10821411

Published by ProQuest LLC (2018). Copyright of the Dissertation is held by the Author.

All rights reserved.

This work is protected against unauthorized copying under Title 17, United States Code Microform Edition (C) ProQuest LLC.

ProQuest LLC.

789 East Eisenhower Parkway

P.O. Box 1346

Ann Arbor, Ml $48106-1346$ 


\begin{abstract}
A class of fractional differential equations are investigated. Using estimates for Mittag-Leffler function and a fixed point theorem, we establish the existence and uniqueness of mild solutions of the equations.
\end{abstract}




\section{Contents}

1 Introduction 1

2 Preliminaries $\quad 9$

3 Mittag-Leffler Function $\quad 35$

4 Fractional Differential Equation $\quad 44$

5 Estimates for $E_{\alpha, \alpha}\left(-\psi(\xi) t^{\alpha}\right) \quad 48$

6 Pseudo-differential Operator with Negative Definite Symbols and Calderón-Vaillancourt Theorem $\quad 55$

7 A fraction-time, spatially invariant, pseudo-differential initial value $\begin{array}{ll}\text { problem } & 67\end{array}$

8 A result for a fractional-time pseudo-differential initial value problem with space-dependent coefficients

9 Existence and Uniqueness for a Class of Stochastic Fractional Dif$\begin{array}{ll}\text { ferential Equations } & \mathbf{7 9}\end{array}$

$\begin{array}{lr}\text { Notations } & 89\end{array}$ 
Bibliography 


\section{Chapter 1}

\section{Introduction}

Fractional calculus is quite an old topic, names such as Leibniz, Euler, Liouville or Weyl, just to mention a few, can all be associated with its development. Their contributions were of pure analytic nature, however application of fractional calculus to problems in mechanics and engineering are well-known. We refer to the monographs of K.B. Oldham and J. Spanier [33], S. Samko et al. [38], A. Kilbas et al. [25], Podlubny [34], and F. Mainardi [28] for introductory material, analytic considerations and applications.

More recently fractional calculus became an important tool in modelling problem in science, engineering and finance related to continuous time random walks. Thus, it became a tool in stochastic analysis. We refer to work done by R. Gorenflo and F. Mainardi [8], [9] and [10], R. Gorenflo et al. [11], R. Hilfer [13], or Y. Zhang et al. [48], to mention some more pioneering or recent work. The very recent monograph [30] by M. Meerschaert gives an excellent introduction to ideas relating fractional calculus and stochastic models.

The basic idea is that continuous time random walks when obeying certain scaling laws are governed by (heavy tail) distributions which satisfy certain fractional differential equations. These could be fractional differential equations with respect to the space variable or to the time variable or even both. Assuming spacial and time homogeneity a Fourier-Laplace transform approach is most appropriate to de- 
rive and solve corresponding equations.

Already in his pioneering paper [44] H. Weyl pointed out relation to (translation invariable) pseudo-differential operators (in case of the torus). Our motivation is to consider first, from the analytic point of view non-trivial equations which are spacially not any more homogeneous, hence a translation invariant pseudo-differential operator is replaced by a pseudo-differential operator having a symbol with variable, i.e. $x$-dependent coefficients.

The pseudo-differential operators we are interested in also link to stochastic processes and where studied by W. Hoh [14], N. Jacob [16]-[20], and N. Jacob and R. Schilling [21], just to give some references.

In our work we aimed to develop mathematical tools, and we have not yet touched problems to modelling:

There are three main results in our work. The first is, Theorem 7.6, which gives conditions for the existence of a mild solution to the problem

$$
{ }_{0} \mathcal{D}_{t}^{\alpha} u(x, t)+\psi\left(D_{x}\right) u(x, t)=g(x, t)
$$

and

$$
\left.{ }_{0} \mathcal{D}_{t}^{\alpha-1} u(x, t)\right|_{t=0}=0
$$

for $g: \mathbb{R} \times[0, \infty) \rightarrow \mathbb{R}$. Here ${ }_{0} \mathcal{D}_{t}^{\alpha}\left(\right.$ and ${ }_{0} \mathcal{D}_{t}^{\alpha-1}$ ) is Riemann-Liouville fractional derivatives and $\psi\left(D_{x}\right)$ is a pseudo-differential operator with symbol $\psi, \psi$ being independent of $x$.

Further, Theorem 8.6, gives for small times and suitable bounds for data the existence of a mild solution to the problem

$$
{ }_{0} \mathcal{D}_{t}^{\alpha} u(x, t)+\psi\left(D_{x}\right) u(x, t)+p\left(x, D_{x}\right) u(x, t)=g(x, t)
$$

with initial data

$$
\left.{ }_{0} \mathcal{D}_{t}^{\alpha-1} u(x, t)\right|_{t=0}=0
$$

Here $p\left(x ; D_{x}\right)$ is a pseudo-differential operator which should be interpreted as a small perturbation of $\psi\left(D_{x}\right)$. 
The third result, Theorem 9.2, gives the existence and uniqueness of mild solution to problem

$$
{ }_{0} \mathcal{D}_{t}^{\alpha} u(x, t)+\psi\left(D_{x}\right) u(x, t)+p\left(x, D_{x}\right) u(x, t)-\int_{0}^{t} f(u(x, \theta)) d B(\theta)=g(x, t),
$$

and

$$
\left.{ }_{0} \mathcal{D}_{t}^{\alpha-1} u(x, t)\right|_{t=0}=0 .
$$

Here $B(\theta)$ is standard Brownian motion and $f$ satisfies a Lipschitz condition and linear growth condition.

Let us start to explain these results in more detail. First we need to explain some notation and auxiliary results.

Though there are some different types on fractional derivatives, but in our paper, we only focus on the Riemann-Liouville fractional derivative ${ }_{0} \mathcal{D}_{t}^{\alpha}$ of the order $\alpha \in$ $(0,1)$. We find in Mainardi [28], Podlubny [34] and Samko [38], that the RiemannLiouville fractional derivatives are defined for $0<\alpha<1$ by,

$$
{ }_{0} \mathcal{D}_{t}^{\alpha} f(t):=\frac{1}{\Gamma(1-\alpha)} \frac{d}{d t} \int_{0}^{t}(t-\tau)^{-\alpha} f(\tau) d \tau,
$$

where

An important method that we use to handle the Riemann-Liouville fractional derivative is Laplace transform, which is given as for $m \in \mathbb{N}$

$$
\mathcal{L}\left\{{ }_{0} \mathcal{D}_{t}^{\alpha} f(t) ; s\right\}=s^{\alpha} F(s)-\sum_{k=0}^{m} d_{k} s^{k-1}, m-1<\alpha \leqslant m,
$$

where $F(s)$ is denoted by $(\mathcal{L} y)(s)$, and

$$
d_{k}=\left(\mathcal{D}_{0+}^{\alpha-k} y\right)(0+), k=1, \ldots, m .
$$

By using the inverse Laplace transform with respect to $s$, we can find a function once we know its Laplace transform.

Next we want to introduce some results on real-valued continuous negative definite functions $\psi$, referring to Hoh [14] and Jacob [18]. We know that $\psi$ has the representation

$$
\psi(\xi)=\int_{\mathbb{R} \backslash\{0\}}(1-\cos y \xi) \nu(d y)
$$


with a Lévy measure $\nu$ satisfying $\int_{\mathbb{R} \backslash\{0\}} 1 \wedge|y|^{2} v(d y)<\infty$. We find further that if $\psi$ is of class $C^{2}$, then for $l \leqslant 2$ and $\psi(\xi) \geqslant 0$

$$
\left|\frac{d^{l} \psi(\xi)}{d \xi^{l}}\right| \leqslant \begin{cases}\psi(\xi), & l=0 \\ C_{1} \psi^{1 / 2}(\xi), & l=1 \\ C_{2}, & l=2\end{cases}
$$

Moreover, for our investigation we require a lower bound for $\psi$, i.e.

$$
\psi(\xi) \geqslant C_{\psi}\left(1+|\xi|^{2}\right)^{\gamma / 2}, C_{\psi}>0
$$

for some $\gamma \in(1,2)$ and all $\xi \in \mathbb{R}$.

Further we need to handle pseudo-differential operators

$$
p\left(x, D_{x}\right) v(x)=(2 \pi)^{-1 / 2} \int_{\mathbb{R}} e^{i x \xi} p(x, \xi) \hat{v}(\xi) d \xi
$$

with symbol $p: \mathbb{R} \times \mathbb{R} \rightarrow \mathbb{R}$ and we require that $\xi \mapsto p(x, \xi)$ is for each $x \in \mathbb{R}$ fixed a continuous negative definite function. Here $\hat{v}$ is denoted by Fourier transform of $v$. We want $p\left(x, D_{x}\right)$ to satisfy the estimate

$$
\left\|p\left(x, D_{x}\right) u(\cdot, t)\right\|_{L^{2}} \leqslant \mu\|u(\cdot, t)\|_{L^{2}}, u(\cdot, t) \in L^{2}(\mathbb{R}),
$$

where $\mu$ is independent of $t$, and

$$
\|u(\cdot, t)\|_{L^{2}}^{2}=\int_{\mathbb{R}}|u(x, t)|^{2} d x .
$$

The most important auxiliary results, are estimates for $E_{\alpha, \alpha}\left(-\psi(\xi) t^{\alpha}\right)$ for $0<$ $\alpha<1$ where $E_{\alpha, \alpha}$ is a generalized Mittag-Leffler function, which is defined by $E_{\alpha, \alpha}:=\sum_{n=0}^{\infty} \frac{\left(-\psi(\xi) t^{\alpha}\right)^{n}}{\Gamma(\alpha n+\alpha)}$. We explain that $E_{\alpha, \alpha}\left(-\psi(\xi) t^{\alpha}\right)$ for $t>0$ belongs in $L^{1}(\mathbb{R})$ and $E_{\alpha, \alpha}\left(-\psi(\xi) t^{\alpha}\right) \leqslant C t^{-\alpha}$. Further we can obtain that $E_{\alpha, \alpha}\left(-\psi(\xi) t^{\alpha}\right)$ belongs in $L^{1}(\mathbb{R}) \cap L^{\infty}(\mathbb{R})$. By proving

$$
\left|\frac{d^{2}}{d \xi^{2}} E_{\alpha, \alpha}\left(-\psi(\xi) t^{\alpha}\right)\right| \leqslant \tilde{C}_{\alpha, \psi} \frac{1}{\left(1+|\xi|^{2}\right)^{\gamma / 2}} \frac{1}{\left(1+t^{\alpha}\right)}
$$

we find the estimate

$$
h(t, \tau):=\left\|F^{-1}\left(E_{\alpha, \alpha}\left(-\psi(\cdot)(t-\tau)^{\alpha}\right)\right)\right\|_{L^{1}} \leqslant \kappa_{1}(t-\tau)^{-\alpha / \gamma}+\kappa_{2}\left(1+(t-\tau)^{\alpha}\right)^{-1}
$$


where $F^{-1}$ is denoted by inverse Fourier transform.

Next we find that

$$
t \mapsto \tilde{h}(t)=t^{\alpha-1} h(t, \tau)
$$

is integrable on every finite interval $[0, T]$ and we get

$$
\int_{0}^{T} \tilde{h}(\tau) d \tau \leqslant \frac{\kappa_{1}}{\alpha(\gamma-1)} T^{\alpha(\gamma-1) / \gamma}+\frac{\kappa_{2}}{\alpha} T^{\alpha}
$$

Now, let us return to explain our main results. We investigate the basic equation with non-zero initial data, namely

$$
{ }_{0} \mathcal{D}_{t}^{\alpha} u(x, t)+\psi\left(D_{x}\right) u(x, t)=0
$$

and

$$
\left.{ }_{0} \mathcal{D}_{t}^{\alpha-1} u(x, t)\right|_{t=0}=b(x) .
$$

We can use the method in Podlubny [34] to obtain the unique solution of this problem, where an approach using a Fourier transform with respect to the space variable(s) and Laplace transform with respect to the time variable is the main tool for solving such an equation. Assuming for $\psi$ with $\gamma \in(1,2)$

$$
\psi(\xi) \geqslant C_{\psi}\left(1+|\xi|^{2}\right)^{\gamma / 2}
$$

we get $E_{\alpha, \alpha}\left(-\psi(\cdot) t^{\alpha}\right) \in L^{1}(\mathbb{R}) \cap L^{\infty}(\mathbb{R})$. However, there is a problem with the smoothness of $b \in \mathcal{S}(\mathbb{R})$.

We next discuss the problem with a non-trivial right-hand-side:

$$
{ }_{0} \mathcal{D}_{t}^{\alpha} u(x, t)+\psi\left(D_{x}\right) u(x, t)=g(x, t)
$$

and

$$
\left.{ }_{0} \mathcal{D}_{t}^{\alpha-1} u(x, t)\right|_{t=0}=b(x)
$$

We find the mild solutions of this problem when the initial data is equal to zero and $g \in L^{\infty}\left([0, T] ; L^{2}(\mathbb{R})\right)$.

Further we prove that the existence and uniqueness of mild solution of the problem

$$
{ }_{0} \mathcal{D}_{t}^{\alpha} u(x, t)+\psi\left(D_{x}\right) u(x, t)+p\left(x, D_{x}\right) u(x, t)=g(x, t)
$$


and

$$
\left.{ }_{0} \mathcal{D}_{t}^{\alpha-1} u(x, t)\right|_{t=0}=0
$$

To get our result, we want to apply Banach's fixed point theorem to the operator

$$
\begin{aligned}
& L_{t} u(x, t):= \\
& (2 \pi)^{-1 / 2} \int_{\mathbb{R}} \int_{0}^{t}(t-\tau)^{\alpha-1} g(x-y, \tau)\left(F^{-1}\left(E_{\alpha, \alpha}\left(-\psi(\cdot)(t-\tau)^{\alpha}\right)\right)\right)(y) d \tau d y \\
& +(2 \pi)^{-1 / 2} \int_{\mathbb{R}} \int_{0}^{t}(t-\tau)^{\alpha-1}\left(p\left(x, D_{x}\right) u(x-y, \tau)\right)\left(F^{-1}\left(E_{\alpha, \alpha}\left(-\psi(\cdot)(t-\tau)^{\alpha}\right)\right)\right)(y) d \tau d y,
\end{aligned}
$$

and prove that this operator is contractive. Moreover, we prove that this operator $L_{t}$ leaves a set invariant and get the desired result.

Finally, we consider a stochastic perturbation of the initial equation:

$$
{ }_{0} \mathcal{D}_{t}^{\alpha} u(x, t)+\psi\left(D_{x}\right) u(x, t)+p\left(x, D_{x}\right) u(x, t)-\int_{0}^{t} f(u(x, \theta)) d B(\theta)=g(x, t),
$$

where $B(\theta)$ is a standard one dimensional Brownian motion and $f$ satisfies a Lipschitz condition and a linear growth condition. Existence and uniqueness for this problem is proved using an adaptation of standard techniques which is possible once we have solved the deterministic problem.

Let us briefly describe the content of each chapter.

In the second Chapter we collect several auxiliary results. We introduce basic notation, for example function spaces and norms, discuss the Fourier transform as well as the Laplace transform, and in particular we discuss the convolution theorem. Positive and negative definite functions in the sense of Bochner and Schoenberg are introduced, their properties treated and relations to convolution semigroups of measures discussed. We also provide several examples of continuous negative definite functions and we discuss the Lévy-Khintchine formula. Subordination in the sense of Bochner is an important tool for us, so we introduce Bernstein functions and subordinate convolution semigroups. Next we turn to one-parameter operator semigroups, in particular to Feller semigroups and sub-Markovian semigroups, and we consider subordinate operator semigroups. Eventually we discuss the BurkholderDavis-Gundy inequality as a probabilistic tool needed later on. 
In Chapter 3 we recollect basic results about two parameter Mittag-Leffler functions. In particular we concentrate on integral representations and the asymptotic behaviour. The latter becomes important when estimating later on terms such as $E_{\alpha, \alpha}\left(-\psi(\xi) t^{\alpha}\right)$

Next, in Chapter 4, we turn to fractional differential equations. We explain the relevant notions and recollect how to solve certain initial value problems with the help of generalized Mittag-Leffler functions. This will allow us to solve equations which are time-fractional and space with respect to pseudo differential equations with constant coefficient. The basic idea is that the Fourier transform will transfer such an equation to a parameter-dependent ordinary time-fractional differential equation. This equation we can solve and now we need to take the inverse Fourier transform of term such as $E_{\alpha, \alpha}\left(-\psi(\cdot) t^{\alpha}\right) * \hat{g}(\cdot, t)$. To proceed forward we need precise estimates for $E_{\alpha, \alpha}\left(-\psi(\cdot) t^{\alpha}\right)$ and some of its derivatives. This is done in Chapter 5 which in some sense is the technical core of our work.

The results up to Chapter 5 already allow to solve some problems leading to Theorem 7.6. However to handle equations with a symbol leading to a pseudodifferential operator with variable coefficients instead of $\psi(D)$, we need more preparations. Chapter 6 is devoted to these preparations. Since we want to stay in the context of pseudo-differential operators with continuous negative symbols - only these operators may generate stochastic processes - we can not apply the standard theory of pseudo-differential operators. Instead we are dependent on work of Hoh [14] and Jacob [17]-[20]. These results are summarized in Chapter 6, not in full generally, but fit for our purpose. In particular we concentrate ourselves on $L^{2}$-bounded operators, i.e. using a Calderón-Vaillancourt result.

The final 3 chapters are devoted to derivation of the main results as described above.

I am glad to thank Professor Niels Jacob, who initialized this work. Both in the studying and writing processes, he gave me a great number of professional suggestions and helps in order to make me finish this dissertation successfully. 
I am grateful to Dr. Chenggui Yuan for his patient guidance and continuous support. He gave me meticulous helps all the time.

And I wish to thank my parents for their support and help. 


\section{Chapter 2}

\section{Preliminaries}

In this chapter we collect some basic background material needed later on. As general reference for our background material may serve [14], [18], as well as [21].

At the beginning, we want to give definitions of some function spaces:

Definition 2.1. Let $G \subset \mathbb{R}^{n}$ be an open set and $m \in \mathbb{N}_{0} \cup\{\infty\}$. We define the spaces:

1

$$
C^{k}(G):=\{u: G \rightarrow \mathbb{C} \mid u \text { is } k-\text { times continuously differentiable }\}
$$

2

$$
C_{0}^{k}(G):=\left\{u \in C^{k}(G) \mid \text { supp } u \text { is compact }\right\}
$$

3

$$
C_{b}^{k}(G):=\left\{u \in C^{k}(G) \mid \partial^{\alpha} u \text { is bounded for }|\alpha| \leqslant k\right\} .
$$

In case $m=0$, we write $C_{b}(G)$ only. In $C_{b}^{k}(G)$ we have the norm

$$
\|u\|_{C_{b}^{k}}:=\sum_{|\alpha| \leqslant k}\left\|\partial^{\alpha} u\right\|_{\infty}
$$


where

$$
\|u\|_{\infty}:=\|u\|_{C_{b}}:=\sup _{x \in \mathbb{R}}|u(x)| .
$$

4

$$
C^{\infty}(G):=\bigcap_{k \in \mathbb{N}} C^{k}(G)
$$

5

$$
C_{0}^{\infty}(G):=\bigcap_{k \in \mathbb{N}} C_{0}^{k}(G)
$$

Definition 2.2. The Schwartz space $\mathcal{S}\left(\mathbb{R}^{n}\right)$ is the function space

$$
\mathcal{S}\left(\mathbb{R}^{n}\right):=\left\{f \in C^{\infty}\left(\mathbb{R}^{n}\right) \mid\|f\|_{\alpha, \beta}<\infty, \text { for all } \alpha, \beta \in \mathbb{N}_{0}^{n}\right\}
$$

where

$$
\|f\|_{\alpha, \beta}=\sup _{x \in \mathbb{R}^{n}}\left|x^{\alpha} \partial^{\beta} f(x)\right|
$$

Definition 2.3. Define the topological dual space $\mathcal{D}^{\prime}\left(\mathbb{R}^{n}\right)$ of $C_{0}^{\infty}\left(\mathbb{R}^{n}\right)$ to be the space of distributions on $\mathbb{R}^{n}$. The topological dual space $\mathcal{S}^{\prime}\left(\mathbb{R}^{n}\right)$ of the Schwartz space $\mathcal{S}\left(\mathbb{R}^{n}\right)$ is called the space of tempered distributions. It consists of all distributions $u \in \mathcal{D}^{\prime}(\mathbb{R})$ having a continuous extension to $\mathcal{S}\left(\mathbb{R}^{n}\right)$, i.e. $\mathcal{S}^{\prime}\left(\mathbb{R}^{n}\right) \subset \mathcal{D}^{\prime}\left(\mathbb{R}^{n}\right)$.

Definition 2.4. On $\mathbb{R}^{n}$ we take the Borel $\sigma$-field and consider Borel measures $\mu$. For $1 \leqslant p<\infty$ we define $L^{p}\left(\mathbb{R}^{n}, \mu\right)$ to be the usual Lebesgue spaces of measurable functions $u: \mathbb{R}^{n} \rightarrow \mathbb{C}$ with finite norm

$$
\|u\|_{L^{p}\left(\mathbb{R}^{n}\right)}:=\left(\int_{\mathbb{R}^{n}}|u(x)|^{p} \mu(d x)\right)^{1 / p} .
$$

Further we define the norm

$$
\|u\|_{L^{\infty}\left(\mathbb{R}^{n}\right)}:=e s s \sup _{x \in \mathbb{R}^{n}}|u(x)| .
$$


We are mainly working with real-valued functions, however the definitions of the function spaces we are using are valid for real as well as complex-valued functions.

Definition 2.5. We define

$$
L^{\infty}\left([0, T] ; L^{2}\left(\mathbb{R}^{n}\right)\right):=\left\{u:[0, T] \rightarrow L^{2}\left(\mathbb{R}^{n}\right) \mid \sup _{0 \leqslant t \leqslant T}\|u(\cdot, t)\|_{L^{2}}<\infty\right\},
$$

which is equipped with norm

$$
\begin{aligned}
\|u\|_{L^{\infty}\left([0, T] L^{2}\left(\mathbb{R}^{n}\right)\right)} & =\sup _{0 \leqslant t \leqslant T}\|u\|_{L^{2}\left(\mathbb{R}^{n}\right)} \\
& =\sup _{0 \leqslant t \leqslant T}\left(\int_{\mathbb{R}^{n}}|u(x, t)|^{2} d x\right)^{1 / 2} .
\end{aligned}
$$

Definition 2.6. The Fourier transform of $f \in \mathcal{S}\left(\mathbb{R}^{n}\right)$ is defined by

$$
F[f](\xi)=\hat{f}(\xi):=(2 \pi)^{-n / 2} \int_{\mathbb{R}^{n}} e^{-i x \xi} f(x) d x, \xi \in \mathbb{R}^{n},
$$

with its inverse Fourier transform, for $g \in \mathcal{S}\left(\mathbb{R}^{n}\right)$,

$$
F^{-1}[g](x):=(2 \pi)^{-n / 2} \int_{\mathbb{R}^{n}} e^{i x \xi} g(\xi) d \xi, x \in \mathbb{R}^{n} .
$$

Definition 2.7. We define the Fourier transform of $f \in L^{1}\left(\mathbb{R}^{n}\right)$ again by (2.1). Further we may define $F^{-1}$ on $L^{1}\left(\mathbb{R}^{n}\right)$ by (2.2). In case that $\hat{f} \in L^{1}\left(\mathbb{R}^{n}\right)$, then $F^{-1}(\hat{f})=f$.

Theorem 2.8. Let $\mu \in \mathcal{M}_{b}^{+}\left(\mathbb{R}^{n}\right)$ be a bounded Borel measure. Its Fourier transform $\hat{\mu}$ is given by

$$
\hat{\mu}=(2 \pi)^{-n / 2} \int_{\mathbb{R}^{n}} e^{-i x \cdot \xi} \mu(d x),
$$

and it is a uniformly continuous function on $\mathbb{R}^{n}$.

Definition 2.9. Let $f \in \mathcal{S}^{\prime}\left(\mathbb{R}^{n}\right)$ and $\phi \in \mathcal{S}\left(\mathbb{R}^{n}\right)$. The Fourier transform $\hat{f}$ of $f$ is defined by

$$
\langle\hat{f}, \phi\rangle:=\langle f, \hat{\phi}\rangle
$$


Because the Fourier transform can not be defined for $u \in L^{2}\left(\mathbb{R}^{n}\right)$, to obtain an extension of the Fourier transform from $L^{1}\left(\mathbb{R}^{n}\right) \cap L^{2}\left(\mathbb{R}^{n}\right)$ to $L^{2}\left(\mathbb{R}^{n}\right)$, we use the Plancherel theorem:

Theorem 2.10. The Fourier transform as it is defined on $L^{1}\left(\mathbb{R}^{n}\right) \cap L^{2}\left(\mathbb{R}^{n}\right)$ has an extension to $L^{2}\left(\mathbb{R}^{n}\right)$. This extension is an isometry on $L^{2}\left(\mathbb{R}^{n}\right)$ which is bijective and has a continuous inverse, in particular we have

$$
\|f\|_{0}=\|\hat{f}\|_{0}
$$

Definition 2.11. We define the convolution of the two functions $f, g \in \mathcal{S}\left(\mathbb{R}^{n}\right)$ by

$$
(f * g)(x):=\int_{\mathbb{R}^{n}} f(x-y) g(y) d y .
$$

If $1 \leqslant p, q, r<\infty$ satisfy $\frac{1}{p}+\frac{1}{q}=\frac{1}{r}+1$, Young's inequality holds

Theorem 2.12. Let $f \in L^{p}\left(\mathbb{R}^{n}\right)$ and $g \in L^{q}\left(\mathbb{R}^{n}\right)$. We have

$$
\|f * g\|_{r} \leqslant\|f\|_{p}\|g\|_{q}
$$

Another important theorem is the convolution theorem linking the Fourier transform and convolution:

Theorem 2.13. Let $f, g \in \mathcal{S}\left(\mathbb{R}^{n}\right)$. Then we have

$$
(f \cdot g)^{\wedge}(\xi)=(2 \pi)^{-n / 2}(\hat{f} * \hat{g})(\xi)
$$

and

$$
(f * g)^{\wedge}(\xi)=(2 \pi)^{n / 2} \hat{f}(\xi) \cdot \hat{g}(\xi)
$$

Let $\left(\Omega_{1}, \mathcal{A}_{1}, \mu_{1}\right)$ and $\left(\Omega_{2}, \mathcal{A}_{2}, \mu_{2}\right)$ to be two measure spaces. We denote by $\mu_{1} \otimes \mu_{2}$ the product measure of $\mu_{1}$ and $\mu_{2}$. The mapping $A_{k}: \mathbb{R}^{n k} \rightarrow \mathbb{R}^{n},\left(x_{1}, \ldots, x_{k}\right) \mapsto$ $x_{1}+\ldots+x_{k}, k \in \mathbb{N}$ is used to define the convolution of measures 
Definition 2.14. Let $\mu_{j} \in \mathcal{M}_{b}^{+}\left(\mathbb{R}^{n}\right), 1 \leqslant j \leqslant k$, be measures. The image of $\mu_{1} \otimes \ldots \otimes \mu_{k}$ under $A_{k}$ is called the convolution of measures and is denoted by

$$
\mu_{1} * \ldots * \mu_{k}:=A_{k}\left(\mu_{1} \otimes \ldots \otimes \mu_{k}\right)
$$

The next theorem collects some properties of measures.

Theorem 2.15. A. For $\mu, \nu \in \mathcal{M}_{b}^{+}\left(\mathbb{R}^{n}\right)$, the convolution theorem holds, i.e.

$$
(\mu * \nu)^{\wedge}=(2 \pi)^{n / 2} \hat{\mu} \cdot \hat{\nu}
$$

B. For $\mu \in \mathcal{M}_{b}^{+}\left(\mathbb{R}^{n}\right)$ and $\nu \in \mathcal{M}_{b}^{+}\left(\mathbb{R}^{m}\right)$ we have

$$
(\mu \otimes \nu)^{\wedge}(\xi, \eta)=\hat{\mu}(\xi) \cdot \hat{\nu}(\eta), \xi \in \mathbb{R}^{n} \text { mboxand } \eta \in \mathbb{R}^{m}
$$

Definition 2.16. Let $f \in L_{\text {loc }}^{1}(\mathbb{R})$, supp $f \subset[0, \infty)$. Its Laplace transform $\mathcal{L}(f)$ is defined by

$$
\mathcal{L}(f)(z):=\int_{0}^{\infty} e^{-z t} f(t) d t, z \in \mathbb{C}
$$

Definition 2.17. Let $\mu$ be a measure on $\mathbb{R}$ and assume that supp $\mu \subset[0, \infty)$ as well as $\int_{0}^{\infty} e^{-x s} \mu(d s)<\infty$ for all $x>0$. Its Laplace transform is defined by

$$
\mathcal{L}(\mu)(z):=\int_{0}^{\infty} e^{-z t} \mu(d t)
$$

Obviously to let the integral (2.13) converge, we need conditions. Let $z=x+i y$, we have

$$
\left|e^{-z t} u(t)\right|=e^{-x t}|u(t)|
$$

It follows that the convergence of the integral (2.13) depends only on $x=\operatorname{Re} z$. Further, when the integral converges for $\operatorname{Re} \tilde{z}=\tilde{x}$, it converges for all $z=x+i y$ such that $\operatorname{Re} z=x \geqslant \tilde{x}$ since

$$
\left|e^{-z t} u(t)\right|=e^{-x t}|u(t)|=e^{-(x-\tilde{x}) t} e^{-\tilde{x} t}|u(t)| \leqslant e^{-\tilde{x} t}|u(t)|
$$


We conclude that there exists a number $x_{0} \in \overline{\mathbb{R}}=[-\infty, \infty]$ such that $t \mapsto e^{-z t} u(t)$ belongs to $L^{1}\left(\overline{\mathbb{R}}_{+}\right)$for $\operatorname{Re} z>x_{0}$, and for $\operatorname{Re} z<x_{0}$ this function does not belong to $L^{1}\left(\tilde{\mathbb{R}}_{+}\right)$. Here we use the convention that $x_{0}=-\infty$ when $t \mapsto e^{-z t} u(t)$ belongs to $L^{1}\left(\overline{\mathbb{R}}_{+}\right)$for all $z \in \mathbb{C}$, and $x_{0}=+\infty$ when there is no $x_{0} \in \mathbb{R}$ such that $t \mapsto e^{-x_{0} t} u(t)$ is an element of $L^{1}\left(\overline{\mathbb{R}}_{+}\right)$. This number $x \in \mathbb{R}$ is called the abscissa of absolute convergence of the Laplace transform of $u$.

We have the convolution theorem for the Laplace transform:

Theorem 2.18. Let $f, g \in L_{\text {loc }}^{1}\left(\mathbb{R}^{n}\right)$. The function $f * g$ has $x_{0} \vee x_{1}, x_{0}, x_{1} \in \mathbb{R}^{n}$, as its abscissa of absolute convergence for the Laplace transform and

$$
\mathcal{L}(f * g)(z)=\mathcal{L}(f)(z) \mathcal{L}(g)(z)
$$

holds for all $z \in \mathbb{C}, \operatorname{Re} z>x_{0} \vee x_{1}$.

Definition 2.19. A family $\left(\mu_{t}\right)_{t \geqslant 0}$ of bounded Borel measures on $\mathbb{R}^{n}$ is called convolution semigroup on $\mathbb{R}^{n}$ if the following conditions are fulfilled

$$
\begin{aligned}
& \mu_{t}\left(\mathbb{R}^{n}\right) \leqslant 1 \text { for all } t \geqslant 0 \\
& \mu_{s} * \mu_{t}=\mu_{t+s}, s, t \geqslant 0 \text { and } \mu_{0}=\varepsilon_{0} \\
& \mu_{t} \rightarrow \varepsilon_{0} \text { vaguely as } t \rightarrow 0 .
\end{aligned}
$$

Definition 2.20. A function $u: \mathbb{R}^{n} \rightarrow \mathbb{C}$ is called positive definite if for any choice of $k \in \mathbb{N}$ and vectors $\xi^{1}, \ldots, \xi^{k} \in \mathbb{R}^{n}$ the matrix $\left(u\left(\xi^{j}-\xi^{l}\right)\right)_{j, l=1, \ldots, k}$ is positive Hermitian, i.e. for all $\lambda_{1}, \ldots, \lambda_{k} \in \mathbb{C}$ we have

$$
\sum_{j, l=1}^{k} u\left(\xi^{j}-\xi^{l}\right) \lambda_{j} \bar{\lambda}_{l} \geqslant 0 .
$$

Now we denote the set of all positive definite functions on $\mathbb{R}^{n}$ by $P\left(\mathbb{R}^{n}\right)$ and $C P\left(\mathbb{R}^{n}\right)$ is the set of all continuous positive definite functions on $\mathbb{R}^{n}$.

We want to state the theorem of Bochner: 
Theorem 2.21. A function $u: \mathbb{R}^{n} \rightarrow \mathbb{C}$ is the Fourier transform of a measure $\mu \in \mathcal{M}_{b}^{+}\left(\mathbb{R}^{n}\right)$ with total mass $\|\mu\|$, if and only if the following conditions are fulfilled

1. $u(0)=\mu \hat{(0)}=(2 \pi)^{-n / 2}\|\mu\| ;$

2. $u$ is positive definite.

Lemma 2.22. For any $u \in P\left(\mathbb{R}^{n}\right)$ we have

$$
|u(\xi)-u(\eta)|^{2} \leqslant 2 u(0)(u(0)-\operatorname{Re} u(\xi-\eta))
$$

and when $u(0)=1$ we get in addition

$$
|u(\xi+\eta)-u(\xi) u(\eta)|^{2} \leqslant\left(1-|u(\xi)|^{2}\right)\left(1-|u(\eta)|^{2}\right)
$$

Let $\left(\mu_{t}\right)_{t \geqslant 0}$ be a convolution semigroup on $\mathbb{R}^{n}$. It follows that the familly $\left(\hat{\mu}_{t}\right)_{t \geqslant 0}$ of the Fourier transform of $\mu_{t}, t \geqslant 0$, consists of continuous positive definite functions on $\mathbb{R}^{n}$ satisfying $\left|\hat{\mu}_{t}(\xi)\right| \leqslant(2 \pi)^{-n / 2}$.

Definition 2.23. A function $\psi: \mathbb{R}^{n} \rightarrow \mathbb{C}$ is called negative definite if

$$
\psi(0) \geqslant 0
$$

and

$$
\xi \mapsto(2 \pi)^{-n / 2} e^{-t \psi(\xi)} \text { is positive definite for } t \geqslant 0 \text {. }
$$

We denote the class of the negative definite functions by $N\left(\mathbb{R}^{n}\right)$. Further we define the continuous negative definite function

$$
C N\left(\mathbb{R}^{n}\right):=N\left(\mathbb{R}^{n}\right) \cap C\left(\mathbb{R}^{n}\right)
$$

Two examples of continuous negative definite functions are, for $0<\alpha \leqslant 1$

$$
\xi \mapsto|\xi|^{2 \alpha},|\xi| \in \mathbb{R}^{n}
$$


and

$$
\xi \mapsto(i \xi)^{\alpha}=|\xi|^{\alpha} \exp \left(i \alpha \frac{\pi}{2}\right), \xi \in \mathbb{R}
$$

We collect some properties of negative definite functions, compare [3]

Proposition 2.24. A function $\psi: \mathbb{R}^{n} \rightarrow \mathbb{C}$ belongs to the class $N\left(\mathbb{R}^{n}\right)$ if for any choice of $k \in \mathbb{N}$ and vectors $\xi^{1}, \ldots, \xi^{k} \in \mathbb{R}^{n}$ the matrix

$$
\left(\psi\left(\xi^{j}\right)+\overline{\psi\left(\xi^{l}\right)}-\psi\left(\xi^{j}-\xi^{l}\right)\right)_{j, l=1, \ldots, k}
$$

is positive Hermitian.

Corollary 2.25. Let $u: \mathbb{R}^{n} \rightarrow \mathbb{C}$ be a positive definite function. Then the function $\xi \mapsto u(0)-u(\xi)$ is in $N\left(\mathbb{R}^{n}\right)$.

Proposition 2.26. Let $\varphi, \varphi_{1}: \mathbb{R}^{n} \mapsto \mathbb{C}$ and $\varphi_{2}: \mathbb{R}^{m} \mapsto \mathbb{C}$ be negative definite functions. Then

1. $\varphi(-\xi)=\overline{\varphi(\xi)}$ for all $\xi \in \mathbb{R}^{n}$

2. $\varphi(0) \in \mathbb{R}$ and $\boldsymbol{R e}(\varphi(\xi)) \geqslant \varphi(0) \geqslant 0$ for all $\xi \in \mathbb{R}^{n}$;

3. The function $\lambda \varphi+\mu \varphi_{1}$ is negative definite for all $\lambda, \mu \geqslant 0$;

4. The function $\lambda \varphi+\mu$ is negative definite for all $\lambda \geqslant 0$ and $\mu \geqslant-\lambda \varphi(0)$;

5. The function $\varphi_{1}+\varphi_{2}:(\xi, \eta) \mapsto \varphi_{1}(\xi)+\varphi_{2}(\eta)$ is negative definite on $\mathbb{R}^{n} \times \mathbb{R}^{m}$;

6. The function

$$
\xi \mapsto(\psi(\xi))^{\alpha}:= \begin{cases}0, & \varphi(\xi)=0 \\ |\varphi(\xi)|^{\alpha} \exp (i \alpha \arg (\varphi(\xi))), & \varphi \neq 0\end{cases}
$$


with $\arg (\varphi(\xi)) \in\left[-\frac{\pi}{2}, \frac{\pi}{2}\right]$ is negative definite for each $\alpha \in[0,1]$.

Lemma 2.27. For any locally bounded negative definite function $\psi \in N\left(\mathbb{R}^{n}\right)$ there exists a constant $c_{\psi}>0$ such that for all $\xi \in \mathbb{R}^{n}$

$$
|\psi(\xi)| \leqslant c_{\psi}\left(1+|\xi|^{2}\right)^{\gamma / 2}, \gamma \in(0,2)
$$

Next, we want to give serval examples on the continuous negative definite functions.

Example 2.28. Any non-negative symmetric quadratic form $q: \mathbb{R}^{n} \times \mathbb{R}^{n} \rightarrow \mathbb{R}$ is a continuous negative definite function. Note, that we do not assume $q$ to have full rank. A convolution semigroup with $q$ as corresponding continuous negative definite function is called a Gaussian semigroup.

Proof. We write $q(\xi)$ instead of $q(\xi, \xi)$ and find for $\xi, \eta \in \mathbb{R}^{n}$ that

$$
2 q(\xi)+2 q(\eta)=q(\xi+\eta)+q(\xi-\eta)
$$

hence $q(0)=0$ and $q(\xi)=q(-\xi)$. Since $q(\xi) \in \mathbb{R}$ we have $q=\bar{q}$. Let us consider the bilinear form $r: \mathbb{R}^{n} \times \mathbb{R}^{n} \rightarrow \mathbb{R}$

$$
r(\xi, \eta)=q(\xi)+q(\eta)-q(\xi-\eta)
$$

It follows that

$$
r(\xi, \xi)=2 q(\xi)-q(0) \geqslant 0
$$

and

$$
\begin{aligned}
r(\xi, \eta) & =q(\xi)+q(\eta)-q(\xi-\eta) \\
& =q(\eta)+q(\xi)-q(\eta-\xi) \\
& =r(\eta, \xi)
\end{aligned}
$$


For $c \in \mathbb{C}$ define $r(c \xi, \eta)=\operatorname{cr}(\xi, \eta)$ and $r(\xi, c \eta)=\operatorname{cr}(\xi, \eta)$. In particular, we have $r(c \xi, \bar{c} \xi)=|c|^{2} r(\xi, \xi) \geqslant 0$. Now, let $k \in \mathbb{N}, \xi^{1}, \ldots, \xi^{k} \in \mathbb{R}^{n}$ and $c_{1}, \ldots, c_{k} \in \mathbb{C}$. For $\xi=\sum_{j=1}^{k} c_{j} \xi^{j}$ we get

$$
\begin{aligned}
0 & \leqslant r\left(\sum_{j=1}^{k} c_{j} \xi^{j}, \sum_{l=1}^{k} \overline{c_{l}} \xi^{l}\right) \\
& =\sum_{j=1}^{j} \sum_{l=1}^{l} c_{j} \overline{c_{l}} r\left(\xi^{j}, \xi^{l}\right) \\
& =\sum_{j, l=1}^{k}\left(q\left(\xi^{j}\right)+q\left(\xi^{l}\right)-q\left(\xi^{j}-\xi^{l}\right)\right) c_{j} \overline{c_{l}} \\
& =\sum_{j, l=1}^{k}\left(q\left(\xi^{j}\right)+\overline{q\left(\xi^{l}\right)}-q\left(\xi^{j}-\xi^{l}\right)\right) c_{j} \overline{c_{l}}
\end{aligned}
$$

thus $q$ is a negative definite function and obviously continuous.

Example 2.29. Let $l: \mathbb{R}^{n} \rightarrow \mathbb{R}$ be a linear functional and define $\psi(\xi):=i l(\xi)$. Then the function $\psi$ is a continuous negative definite function. Moreover, whenever $\psi(\xi)=i l(\xi), l: \mathbb{R}^{n} \rightarrow \mathbb{R}$, is a continuous negative definite function, then $l$ must be linear.

Proof. For $k \in \mathbb{N}$ and $\xi^{1}, \ldots, \xi^{k} \in \mathbb{R}^{n}$ it follows that

$$
\begin{aligned}
& \psi\left(\xi^{j}\right)+\overline{\psi\left(\xi^{j^{\prime}}\right)}-\psi\left(\xi^{j}-\xi^{j^{\prime}}\right) \\
& =i l\left(\xi^{j}\right)+i \overline{l\left(\xi^{j^{\prime}}\right)}-i l\left(\xi^{j}-\xi^{j^{\prime}}\right) \\
& =i\left(l\left(\xi^{j}\right)-l\left(\xi^{j^{\prime}}\right)-l\left(\xi^{j}-\xi^{j^{\prime}}\right)\right) \\
& =0
\end{aligned}
$$

Since any linear functional on $\mathbb{R}^{n}$ is of the form $l(\xi)=h \cdot \xi$ with some $h \in \mathbb{R}^{n}$, it follows that for any $h \in \mathbb{R}^{n}$ the function $\xi \mapsto i h \cdot \xi$ belongs to $C N\left(\mathbb{R}^{n}\right)$.

To proof the second statement assume that $\psi(\xi)=i l(\xi), l: \mathbb{R}^{n} \rightarrow \mathbb{R}$, is a continuous negative definite function. Then the function $\phi_{t}(\xi)=e^{-t \psi(\xi)}, t>0$, is positive definite and $\left|\phi_{t}(\xi)\right|=1$. Therefore, by (2.17) we have

$$
e^{i t l(\xi+\eta)}=e^{-t \psi(\xi+\eta)}=e^{-t \psi(\xi)} e^{-t \psi(\eta)}=e^{-i t l(\xi)} e^{-i t l(\eta)}=e^{-i t(l(\xi)+l(\eta))}
$$


and for $k \in \mathbb{N}$ it follows that

$$
\left(e^{-i t \frac{1}{k} l(\xi)}\right)^{k}=e^{-i t l\left(\frac{\xi}{k}+\ldots+\frac{\xi}{k}\right)}=\left(e^{-i t l\left(\frac{\xi}{k}\right)}\right)^{k},
$$

implying that $l$ is additive and $l\left(\frac{m}{k} \xi\right)=\frac{m}{k} l(\xi)$ for all $\frac{m}{k} \in \mathbb{Q}$. The continuity of $l$ implies now that $l$ must be a linear functional on $\mathbb{R}^{n}$.

Example 2.30. Since for $h \in \mathbb{R}^{n}$ the function $\xi \mapsto e^{-i h \cdot \xi}$ is positive definite and $e^{0}=1$, it follows from Corollary 2.25 that $\xi \mapsto\left(1-e^{-i h \cdot \xi}\right)$ is a continuous negative definite function implying that $\xi \mapsto(1-\cos (h \cdot \xi))$ is an element in $C N\left(\mathbb{R}^{n}\right)$ too. For $h \in \mathbb{R}, h \leqslant 0$, and $t \leqslant 0$ let us consider on $\mathbb{R}$ the measures

$$
\mu_{t}=\sum_{k=0}^{\infty} e^{-t} \frac{t^{k}}{k !} \varepsilon_{h k} .
$$

Taking the Fourier transform of $\mu_{t}$ we get

$$
\begin{aligned}
\hat{\mu}_{t}(\xi) & =\sum_{k=0}^{\infty} e^{-t} \frac{t^{k}}{k !} \hat{\varepsilon}_{h k} \\
& =\sum_{k=0}^{\infty} e^{-t} \frac{t^{k}}{k !}(2 \pi)^{-1 / 2}\left\langle\varepsilon_{h k}, e^{-i(\cdot, k)}\right\rangle \\
& =(2 \pi)^{-1 / 2} e^{-t} \sum_{k=0}^{\infty} \frac{t^{k}}{k !} e^{-i k h \xi} \\
& =(2 \pi)^{-1 / 2} e^{-t} \sum_{k=0}^{\infty} \frac{\left(t \cdot e^{-i h \xi}\right)}{k !} \\
& =(2 \pi)^{-1 / 2} e^{-t\left(1-e^{-i h \xi}\right)}
\end{aligned}
$$

implying that $\xi \mapsto 1-e^{-i h \xi}$ is a continuous negative definite function and that $\left(\mu_{t}\right)_{t \leqslant 0}$ is a continuous semigroup on $\mathbb{R}$, called the Poisson semigroup.

We want to give further examples of continuous negative definite functions, compare [20]:

\section{Example 2.31. Generalized Hyperbolic Distributions}

The density of $X_{1}$ of a generalized hyperbolic process is given by

$P_{G H}(x ; \lambda, \alpha, \beta, \delta, \mu)=a(\lambda, \alpha, \beta, \delta)\left(\delta^{2}+(x-\mu)^{2}\right)^{\frac{\lambda-\frac{1}{2}}{2}} K_{\lambda-\frac{1}{2}}\left(\alpha \sqrt{\delta^{2}+(x-\mu)^{2}}\right) e^{\beta(x-\mu)}$ 
where the normalizing constant is

$$
\alpha(\lambda, \alpha, \beta, \delta)=\frac{\left(\alpha^{2}-\beta^{2}\right)^{1 / 2}}{\sqrt{2 \pi} \alpha^{\lambda-\frac{1}{2}} \delta^{\lambda} K_{\lambda}\left(\delta \sqrt{\alpha^{2}-\eta^{2}}\right)} .
$$

We gives the negative definite function for $\alpha>0,0 \leqslant|\beta|<\alpha, \mu \in \mathbb{R}, \delta>0$ and $\lambda \in \mathbb{R}$,

$$
\psi(\xi)=-i \xi\left(\mu+\frac{\beta \delta^{2} K_{\lambda+1}\left(\delta \sqrt{\alpha^{2}-\beta^{2}}\right)}{K_{\lambda}\left(\delta \sqrt{\alpha^{2}-\beta^{2}}\right)}\right)-\int\left(e^{i x \cdot \xi}-1-i x \cdot \xi\right) g(x) d x
$$

where

$$
g(x)=\frac{e^{\beta x}}{|x|}\left(\int_{0}^{\infty}\left(e^{-\sqrt{2 y+\alpha^{2}}|x|}\right) \frac{1}{\pi^{2} y\left(J_{|\lambda|}^{2}(\delta \sqrt{2 y})+Y_{|\lambda|}^{2}(\delta \sqrt{2 y})\right)} d y+\lambda e_{\chi_{\lambda} \geqslant 0}^{-\alpha|x|}\right) .
$$

\section{Example 2.32. Hyperbolic Distributions}

If we set $\lambda=1$ in the generalized hyperbolic model then we get the hyperbolic model, $X_{1}$ has the density

$$
P_{H}(x)=\frac{\sqrt{\alpha^{2}-\beta^{2}}}{2 \alpha \delta K_{1}\left(\delta \sqrt{\alpha^{2}-\beta^{2}}\right)} e^{-\alpha \sqrt{\delta^{2}+(x-\mu)^{2}}+\beta(x+\mu)} .
$$

We give the negative definite function for $\alpha>0,0 \leqslant|\beta|<\alpha, \mu \in \mathbb{R}$ and $\delta>0$,

$$
\psi(\xi)=-i \xi\left(\mu+\frac{\beta \delta^{2} K_{1+1}\left(\delta \sqrt{\alpha^{2}-\beta^{2}}\right)}{K_{1}\left(\delta \sqrt{\alpha^{2}-\beta^{2}}\right)}\right)-\int\left(e^{i x \cdot \xi}-1-i x \cdot \xi\right) g(x) d x
$$

where

$$
g(x)=\frac{e^{\beta x}}{|x|}\left(\int_{0}^{\infty}\left(e^{-\sqrt{2 y+\alpha^{2}}|x|}\right) \frac{1}{\pi^{2} y\left(J_{1}^{2}(\delta \sqrt{2 y})+Y_{1}^{2}(\delta \sqrt{2 y})\right)} d y e^{-\alpha|x|}\right) .
$$

\section{Example 2.33. Normal Inverse Gaussian Distributions}

We get this class of distributions by setting $\lambda=\frac{1}{2}$ in the generalized hyperbolic distribution. The density of $X_{t}$ is given by

$$
P_{a, b, \mu ; \delta}(x)=\frac{a}{\pi} e^{\delta t \sqrt{a^{2}-b^{2}}+b(x-\mu t)} \frac{K_{1}\left(a \delta t \sqrt{1+\left(\frac{x-\mu t}{\delta}\right)^{2}}\right)}{\sqrt{1+\left(\frac{x-\mu}{\delta t}\right)^{2}}} .
$$


In [1] O. Barndorff-Nielsen gives its Lévy density as

$$
\nu_{N I G}(x)=\frac{\delta a}{\pi} e^{b x} \frac{K_{1}(a|x|)}{|x|} .
$$

The diffusion component is 0 and the drift is given by

$$
\mu_{N I G}=\mu+\frac{2 \delta a}{\pi} \int_{0}^{1} \sinh (b x) K_{1}(a|x|) d x .
$$

The negative definite function is given by

$$
\psi(\xi)=i \mu \xi+\delta\left(\sqrt{a^{2}-(b+i \xi)^{2}}-\sqrt{q^{2}-b^{2}}\right)
$$

for $0 \leqslant|b|<a, \delta>0$ and $m \in \mathbb{R}$.

\section{Example 2.34. Variance Gamma Process}

The Lévy density can be calculated as

$$
k_{V G}(x)= \begin{cases}\frac{C e^{-M x}}{x}, & x>0 \\ \frac{C e^{-G|x|}}{|x|}, & x<0\end{cases}
$$

where $C=\frac{1}{\nu}$,

$$
G=\sqrt{\frac{\theta^{2} \nu^{2}}{4}+\frac{\delta^{2} \nu}{2}}+\frac{\theta \nu}{2}
$$

and

$$
M=\sqrt{\frac{\theta^{2} \nu^{2}}{4}+\frac{\delta^{2} \nu}{2}}-\frac{\theta \nu}{2} .
$$

The negative definite function is given by

$$
\psi(\xi)=\frac{1}{\nu} \ln \left(1-i \theta \nu \xi+\frac{\sigma^{2} \nu}{2} \xi^{2}\right),
$$

for $\theta \geqslant 0, \sigma \geqslant 0$ and $\nu \geqslant 0$.

\section{Example 2.35. Carr-Geman-Madan-Yor process}

The Lévy density has the following form:

$$
k_{V G}(x)= \begin{cases}\frac{C e^{-M x}}{x^{1+Y}}, & x>0 \\ \frac{C e^{-G|x|}}{|x|^{1+Y}}, & x<0\end{cases}
$$

The negative definite function is given by

$$
\psi \xi-C \Gamma(-Y)\left\{(M-i \xi)^{Y}-M^{Y}+(G+i \xi)^{Y}-G^{Y}\right\}
$$

for $C>0, M \geqslant 0, Y$ is not in $\mathbb{Z}, G \geqslant 0$ and $Y<2$. 


\section{Example 2.36. Truncated Lévy Process}

This process is directly constructed using a negative definite function. Including the cases $\nu=0,1$ the negative definite function is given by:

$$
\psi(\nu, \lambda, c)= \begin{cases}\frac{c(\ln (\lambda \pm i \xi)-\ln \lambda)}{2}, & \text { if } \nu=0, \\ -\frac{c}{2 \cos \left(\frac{\pi \nu}{2}\right)}\left(\lambda^{\nu}-(\lambda \pm i \xi)^{\nu}\right), & \text { if } \nu \in(0,1) \cup(1,2), \\ -\frac{c}{\pi}((\lambda \pm i \xi) \ln (\lambda \pm i \xi)-\lambda \ln \lambda), & \text { if } \nu=1,\end{cases}
$$

where $c>0, \lambda>0$. The negative definite function is given by

$$
\psi(\xi)=\frac{c^{\alpha}}{\cos (\pi \alpha / 2)}\left(\left(\xi^{2}+\lambda^{2}\right)^{\alpha / 2} \cos (\alpha \arctan (\xi / \lambda))-\lambda^{\alpha}\right),
$$

for $0<\lambda<2, c>0$ and $\lambda>0$.

\section{Example 2.37. Meixner Process}

The density of $X_{t}$ is given by

$$
p_{(a, b, m, s, t)}(x)=\frac{\left(2 \cos \left(\frac{b}{2}\right)\right)^{2 s t}}{2 a \pi \Gamma(2 s t)} e^{\frac{b(x-m t)}{a}}\left|\Gamma\left(s t+\frac{i(x-m t)}{a}\right)\right|^{2} .
$$

The Lévy measure is

$$
\nu(d x)=s \frac{e^{\frac{b x}{a}}}{x \sinh \left(\frac{p i y}{a}\right)} d y-m,
$$

the drift is

$$
m_{\text {Meixner }}=-a s \tan \left(\frac{b}{2}\right)+2 s \int_{1}^{\infty} \frac{\sinh \left(\frac{b y}{a}\right)}{\sinh \left(\frac{\pi y}{a}\right)} d y-m
$$

and there is no diffusion component. The negative definite function is given by

$$
\psi(\xi)=-i l \xi+2 r\left(\ln \cosh \left(\frac{a \xi-i b}{2}-\ln \cos \left(\frac{b}{2}\right)\right)\right),
$$

for $a>0,-\pi<b<\pi, r>0$ and $l \in \mathbb{R}$.

\section{Example 2.38. Real meixner process}

The density of the transition probability of the real Meixner process is for $b=0$ given by

$$
p_{t}(x)=\frac{4^{s t}}{2 \pi a \Gamma(2 s t)}\left|\Gamma\left(s t+i \frac{x}{a}\right)\right|^{2}
$$


and for $(b \neq 0)$ by

$$
\begin{aligned}
& p_{t}(x)=\frac{\cos ^{2 s t}\left(\frac{b}{2}\right) 4^{s t}}{(2 \pi)^{2} \Gamma(2 s t) a^{2}} \sum_{k=0}^{\infty} \frac{s t(s t+1) \cdot \ldots \cdot(s t+k-1)}{k !} \\
& \quad \times \sin ^{2 k}\left(\frac{b}{2}\right) \frac{4^{k}}{\Gamma(2 k)} \int_{-\infty}^{\infty}\left|\Gamma\left(s t+i \frac{(x-v)}{a}\right)\right|^{2}\left|\Gamma\left(k+i \frac{v}{a}\right)\right|^{2} d v .
\end{aligned}
$$

The negative definite function is given by

$$
\psi(\xi)=\psi_{\operatorname{Rem}}(\xi):=\operatorname{Re} \psi(\xi)=-2 s \ln \cos \left(\frac{b}{2}\right)+s \ln \left(\cosh ^{2}\left(\frac{a \xi}{2}\right)-\sin ^{2}\left(\frac{b}{2}\right)\right)
$$

for $a>0,-\pi<b<\pi$ and $s>0$.

Note we want to discuss by the Lévy-Khinchin formula for continuous negative definite function. The Lévy-Khinchin formula says that every continuous negative definite function $\psi: \mathbb{R}^{n} \rightarrow \mathbb{C}$ has the representation

$$
\psi(\xi)=c+i(d \cdot \xi)+q(\xi)+\int_{\mathbb{R}^{n} \backslash\{0\}}\left(1-e^{-i x \cdot \xi}-\frac{i x \cdot \xi}{1+|x|^{2}}\right) \frac{1+|x|^{2}}{|x|^{2}} \mu(d x)
$$

with a non-negative constant $c \geqslant 0$, a vector $d \in \mathbb{R}^{n}$, a symmetric positive semidefinite quadratic form $q$, and a finite Borel measure $\mu$ on $\mathbb{R}^{n} \backslash\{0\}$. The function $\psi$ is uniquely determined by $(c, d, q, \mu)$ and any such quadruple defines via (2.48) a continuous negative definite function. An analytic proof is given by N. Jacob and R.L. Schilling [21] or [18].

First, we introduce an important definition of the Lévy measure:

Definition 2.39. Let $\mu$ be the measure in the Lévy-Khinchin representation of the continuous negative definite function $\psi: \mathbb{R}^{n} \rightarrow \mathbb{C}$. The measure

$$
\nu(d x)=\frac{1+|x|^{2}}{|x|^{2}} \mu(d x)
$$

defined on $\mathcal{B}\left(\mathbb{R}^{n} \backslash\{0\}\right)$ is called the Lévy measure associated with $\psi$.

Let $\psi: \mathbb{R}^{n} \rightarrow \mathbb{R}$ be a real-valued continuous negative definite function and denote by $\nu$ its Lévy measure. It follows that

$$
\int_{\mathbb{R}^{n} \backslash\{0\}} \frac{|x|^{2}}{1+|x|^{2}} \nu(d x)<\infty
$$


and $\psi$ has the representation

$$
\psi(\xi)=c+q(\xi)+\int_{\mathbb{R}^{n} \backslash\{0\}}(1-\cos (x \cdot \xi)) \nu(d x) .
$$

The following theorem is due to W. Hoh [14]:

Theorem 2.40. Let $\psi: \mathbb{R}^{n} \rightarrow \mathbb{R}$ be a continuous negative definite function with Lévy-Khinchin representation (2.50). Suppose that for $2 \leqslant l \leqslant m$ all absolute moments of the Lévy measure $\nu$ exist, i.e.

$$
M_{t}:=\int_{\mathbb{R}^{n} \backslash\{0\}}|x|^{l} \nu(d x)<\infty, 2 \leqslant l \leqslant m .
$$

Then, $\psi$ is of class $C^{m}\left(\mathbb{R}^{n}\right)$, and for $\alpha \in \mathbb{N}_{0}^{n}, \alpha \leqslant m$, we have the estimate

$$
\left|\partial_{\xi}^{\alpha} \psi(\xi)\right| \leqslant c_{|\alpha|} \cdot \begin{cases}\psi(\xi), & \alpha=0 \\ \psi^{1 / 2}(\xi), & |\alpha|=1 \\ 1, & |\alpha| \geqslant 2\end{cases}
$$

with $c_{0}=1, c_{1}=\left(2 M_{2}\right)^{1 / 2}+2 \lambda^{1 / 2}, c_{2}=M_{2}+2 \lambda$ and $c_{l}=M_{l}, 3 \leqslant l \leqslant m$, where $\lambda$ is the maximal eigenvalue of the quadratic form $q$ in (2.52).

Now Bernstein functions and subordination are investigated.

Definition 2.41. A function $f \in C^{\infty}((0, \infty))$ is called a Bernstein function if

$$
f \geqslant 0 \text { and }(-1)^{k-1} \frac{d^{k} f(x)}{d x^{k}} \geqslant 0
$$

holds for all $k \in \mathbb{N}$.

Definition 2.42. A function $f:(0, \infty) \rightarrow \mathbb{R}$ is a completely monotone function if $f$ is of class $C^{\infty}$ and

$$
(-1)^{n} f^{(n)}(\lambda) \geqslant 0 \text { for all } n \in \mathbb{N} \cup\{0\} \text { and } \lambda>0
$$


Thus, we easily see a Bernstein function is positive, increasing and concave. Furthermore, the set of all Bernstein functions forms a convex cone containing the positive constants. And a function $f$ is a Bernstein function if and only if, $f^{\prime}$ is a completely monotone function.

Proposition 2.43. For a function $f:(0, \infty) \rightarrow \mathbb{R}$ the following two assertions are equivalent:

1. $f$ is a Bernstein function;

2. $f \geqslant 0$ and for all $t>0$ the function $\exp (-t f)$ is completely monotone.

Theorem 2.44. Let $f$ be a Bernstein function. Then there exists constants $a, b \geqslant 0$ and measure $\mu$ on $(0, \infty)$ verifying

$$
\int_{0+}^{\infty} \frac{s}{1+s} \mu(d s), \infty
$$

such that

$$
f(x)=a+b x+\int_{0+}^{\infty}\left(1-e^{-x s}\right) \mu(d s), x>0 .
$$

The triple $(a, b, \mu)$ is uniquely determined by $f$. Conversely, given $a, b \geqslant 0$ and $a$ measure $\mu$ on $(0, \infty)$ satisfying (2.55), the (2.56) defines a Bernstein function.

Corollary 2.45. The convex cone of Bernstein functions is closed under pointwise convergence.

Next we want to study Bernstein functions with convolution semigroups of measures.

Definition 2.46. Let $\left(\eta_{t}\right)_{t \geqslant 0}$ be a convolution semigroup of measures on $\mathbb{R}$. It is said to be supported by $[0, \infty)$ if supp $\eta_{t} \subset[0, \infty)$ for all $t \geqslant 0$. 
Theorem 2.47. Let $f:(0, \infty) \rightarrow \mathbb{R}$ be a Bernstein function. Then there exists $a$ unique convolution semigroup $\left(\eta_{t}\right)_{t \geqslant 0}$ supported by $[0, \infty)$ such that

$$
\mathcal{L}\left(\eta_{t}\right)(x)=e^{t f(x)}, x>0 \text { and } t>0
$$

holds. Conversely, for any convolution semigroup $\left(\eta_{t}\right)_{t \geqslant 0}$ supported by $[0, \infty)$ there exists a unique Bernstein function $f$ such that (2.57) holds.

Remark 2.48. Since (2.55) holds in the half-plane $\boldsymbol{R e} z \geqslant 0$ we have

$$
\eta_{t}([0, \infty])=\mathcal{L}\left(\eta_{t}\right)(0)=e^{-t f(0)}, t>0
$$

Thus $\eta_{t}$ is a probability measure if and only if $f(0)=0$. Further note that the continuous negative definite function associated with the convolution semigroup $\left(\eta_{t}\right)_{t \geqslant 0}$ is given by $u \mapsto f(i y)$. In particular, this means that for any Bernstein function $f$ the function $\xi \mapsto \psi(\xi):=f(i \xi)$ is negative definite and continuous. Form (2.56) we find the Lévy-Khinchin representation of $\psi$ to be

$$
\psi(\xi)=a+b \xi+\int_{0+}^{\infty}\left(1-e^{-i x \xi}\right) \mu(d \xi)
$$

where $\mu$ is the measure from (2.55).

Let $\left(\mu_{t}\right)_{t \geqslant 0}$ be a convolution semigroup on $\mathbb{R}^{n}$ with associated continuous negative definite function $\psi$. Further, let $f$ be a Bernstein function with associated semigroup $\left(\eta_{t}\right)_{t \geqslant 0}$ supported on $[0, \infty)$. For $f$ we have the representation (2.56), and since $\operatorname{Re} \psi \geqslant 0$ we may consider the function $f \circ \psi$ :

$$
(f \circ \psi)(\xi)=a+b \psi(\xi)+\int_{0+}^{\infty}\left(1-e^{-s \psi(\xi)}\right) \mu(d s) .
$$

The function $\xi \mapsto e^{-s \psi(\xi)}$ is positive definite, hence $\xi \mapsto 1-e^{-s \psi(\xi)}$ is negative definite by Corollary 2.25, imply the $f \circ \psi$ is negative definite.

Lemma 2.49. For any Bernstein function $f$ and continuous negative definite function $\psi: \mathbb{R}^{n} \rightarrow \mathbb{C}$, the function $f \circ \psi$ is also continuous and negative definite. 
Proposition 2.50. Let $\psi \in C N\left(\mathbb{R}^{n}\right)$ be a continuous negative definite function with associated convolution semigroup $\left(\mu_{t}\right)_{t \geqslant 0}$ on $\mathbb{R}^{n}$. Further let $f$ be a Bernstein function with associated semigroup $\left(\eta_{t}\right)_{t \geqslant 0}$ supported on $[0, \infty)$. The convolution semigroup $\left(\left(\mu_{t}^{f}\right)_{t \geqslant 0}\right)$ on $\mathbb{R}^{n}$ associated with the continuous negative definite function $f \circ \psi$ is given by

$$
\int_{\mathbb{R}^{n}} \phi(x) \mu_{t}^{f}(d x)=\int_{0}^{\infty} \int_{\mathbb{R}^{n}} \phi(x) \mu_{s}(d x) \eta_{t}(d s), \phi \in C_{0}\left(\mathbb{R}^{n}\right) .
$$

Remark 2.51. Instead of (2.61) we shall write

$$
\mu_{t}^{f}=\int_{0}^{\infty} \mu_{s} \eta_{t}(d s) \text { vaguely. }
$$

Definition 2.52. In the situation of Proposition 2.50 we call the convolution semigroup $\left(\mu_{t}^{f}\right)_{t \geqslant 0}$ ) the semigroup subordinate (in the sense of Bochner) to $\left(\mu_{t}\right)_{t \geqslant 0}$ with respect to $\left(\eta_{t}\right)_{t \geqslant 0}$.

Theorem 2.53. Let $f$ be a function defined on the half-plane $\boldsymbol{R e} z \geqslant 0$ such that for all $n \in \mathbb{N}$ and any $\psi \in C N\left(\mathbb{R}^{n}\right)$ the function $f \circ \psi$ belongs to $C N\left(\mathbb{R}^{n}\right)$ too. Then $f$ is a Bernstein function.

Let the function $\xi \mapsto|\xi|^{2}$ is an element of $C N\left(\mathbb{R}^{n}\right)$ for any $n \in \mathbb{N}$. Therefore, for any Bernstein function $f$ the function $\xi \mapsto f\left(|\xi|^{2}\right)$ is a radial symmetric continuous negative definite function.

Theorem 2.54. If for all $n \in \mathbb{N}$ the function $\xi \mapsto f\left(|\xi|^{2}\right)$ is an element in $C N\left(\mathbb{R}^{n}\right)$, then $f$ is a Bernstein function. 
Lemma 2.55. 1. For every Bernstein function $f$ we have

$$
\frac{f^{\prime} s}{f(s)} \leqslant \frac{1}{s} \text { for } s>0 \text {. }
$$

2. For every Bernstein function $f$ and all $c \geqslant 1$ it follows that

$$
\frac{1}{c} f(s) \leqslant f(c s) \leqslant c f(s) .
$$

3. If $f$ is a complete Bernstein function, then

$$
\left|\frac{f^{(k+1)}(s)}{f^{(k)}(s)}\right| \leqslant \frac{k+1}{s}, s>0,
$$

holds for all $k \in \mathbb{N}$.

4. For the derivatives of any Bernstein function we have

$$
\left|f^{(k)}(s)\right| \leqslant \frac{k !}{s^{k}} f(s), s>0 \text { and } k \in \mathbb{N}_{0} .
$$

Corollary 2.56. Let $f$ and $g$ be two Bernstein functions. Then $f \circ g$ is also $a$ Bernstein function.

We could relate the continuous negative definite function to fractional derivatives. Let $0<\alpha<1$ be fixed. The function $\xi \mapsto( \pm i \xi)^{\alpha}$ are continuous negative definite functions. On $\mathcal{S}(\mathbb{R})$ or $C_{0}^{\infty}(\mathbb{R})$, we consider the operators

$$
u \mapsto(2 \pi)^{-1 / 2} \int_{\mathbb{R}} e^{i(\cdot, \xi)}( \pm i \xi)^{\alpha} \hat{u}(\xi) d \xi
$$

Compare [22], for $u \in \mathcal{S}(\mathbb{R})$, these operators can be rewritten by

$$
\begin{aligned}
D_{+, R}^{\alpha} u(x): & =(2 \pi)^{-1 / 2} \int_{\mathbb{R}} e^{i x \xi}(i \xi)^{\alpha} \hat{u}(\xi) d \xi \\
& =\frac{1}{\Gamma(1-\alpha)} \frac{d}{d x} \int_{-\infty}^{x} \frac{u(y)}{(x-y)^{\alpha}} d y \\
& =\frac{\alpha}{\Gamma(1-\alpha)} \int_{0}^{\infty} \frac{u(x)-u(x-y)}{y^{1+\alpha}} d y
\end{aligned}
$$


and

$$
\begin{aligned}
D_{-, R}^{\alpha} u(x): & =\int_{\mathbb{R}} e^{i x \xi}(-i \xi)^{\alpha} \hat{u}(\xi) d \xi \\
& =-\frac{1}{\Gamma(1-\alpha)} \frac{d}{d x} \int_{x}^{\infty} \frac{u(y)}{(y-x)^{\alpha}} d y \\
& =\frac{\alpha}{\Gamma(1-\alpha)} \int_{0}^{\infty} \frac{u(x)-u(x+y)}{y^{1+\alpha}} d y
\end{aligned}
$$

Now, Let $\left(\mathcal{B},\|\cdot\|_{\mathcal{B}}\right)$ be a real or complex Banach space. We want to introduce the definition of operator semigroup:

Definition 2.57. A one parameter family $\left(T_{t}\right)_{t \geqslant 0}$ of bounded linear operators $T_{t}$ : $\mathcal{B} \rightarrow \mathcal{B}$ is called a (one parameter) semigroup of operator, if $T_{0}=$ id and $T_{s} \circ T_{t}=T_{s+t}$ hold for all $s, t \geqslant 0$.

B. We define the strongly continuous semigroup $\left(T_{t}\right)_{t \geqslant 0}$ for all $u \in \mathcal{B}$ if

$$
\lim _{t \rightarrow 0}\left\|T_{t} u-u\right\|_{\mathcal{B}}=0
$$

C.And we call the semigroup $\left(T_{t}\right)_{t \geqslant 0}$ a contraction semigroup, if for all $t \geqslant 0$

$$
\left\|T_{t}\right\| \leqslant 1
$$

holds, i.e. if each of the operators $T_{t}$ is a contraction.

Definition 2.58. Let $\left(T_{t}\right)_{t \geqslant 0}$ be a strongly continuous semigroup of operators on a Banach space $\left(\mathcal{B},\|\cdot\|_{\mathcal{B}}\right)$. The generator $A$ of $\left(T_{t}\right)_{t \geqslant 0}$ is defined by

$$
A u:=\lim _{t \rightarrow 0} \frac{T_{t} u-u}{t} \text { (strong limit) }
$$

with domain

$$
\mathcal{D}(A):=\left\{u \in \mathcal{B} \mid \lim _{t \rightarrow 0} \frac{T_{t} u-u}{t} \text { exists as strong limit }\right\} .
$$


Proposition 2.59. For each strongly continuous semigroup $\left(T_{t}\right)_{t \geqslant 0}$ on $\mathcal{B}$ there exists a closed operator $A$ with domain $\mathcal{D}(A)$, dense in $\left(\mathcal{B},\|\cdot\|_{\mathcal{B}}\right)$, which is the generator of $\left(T_{t}\right)_{t \geqslant 0}$. For each $t \geqslant 0$ the operator $T_{t}$ maps $\mathcal{D}(A)$ into itself. Conversely, if $(A, \mathcal{D}(A))$ is the operator of a strongly continuous semigroup on $\mathcal{B}$, the $A$ is a closed operator and $\mathcal{D}(A)$ is dense in $\mathcal{B}$.

Definition 2.60. A family $\left(R_{\mu}\right)_{\mu>0}$ of bounded operators on a Banach space $\mathcal{B}$ is called a strongly continuous contraction resolvent if $R_{\mu}-R_{\lambda}=(\lambda-$ $\mu) R_{\mu} R_{\lambda}$ for all $\lambda, \mu>0$, and if the operators $\mu R_{\mu}$ are contractions on $\mathcal{B}$ such that $\lim _{\mu \rightarrow \infty}\left\|\mu R_{\mu} f-f\right\|_{\mathcal{B}}=0$ for each $f \in \mathcal{B}$.

Proposition 2.61. A. For a strongly continuous contraction resolvent $\left(R_{\mu}\right)_{\mu>0}$ there exists a unique linear operator $(A, \mathcal{D}(A))$ on $\mathcal{B}$, densely defined and closed, such that $R_{\mu}=(\mu-A)^{-1}$ for all $\mu>0$.

$B$. Let $\left(T_{t}\right)_{t \geqslant 0}$ be a strongly continuous contraction semigroup on $\mathcal{B}$, with generator $(A, \mathcal{D}(A))$, and for $\mu>0$ let $R_{\mu}^{A}:=(\mu-A)^{-1}$. Then

$$
R_{\mu}^{A} f=\int_{0}^{\infty} e^{-\mu t}\left(T_{t} f\right) d t
$$

for all $\mu>0$ and $f \in \mathcal{B}$. In particular, the family $\left(R_{\mu}^{A}\right)_{\mu>0}$ is a strongly continuous contraction resolvent.

Proposition 2.62. Let $(A, \mathcal{D}(A))$ be the generator of the strongly continuous semigroup $\left(T_{t}\right)_{t \geqslant 0}$ on $\mathcal{B}$ and let $\mathcal{D}_{0} \subset \mathcal{D}(A)$ be a dense subspace of $\mathcal{B}$. If $T_{t}$ maps $\mathcal{D}_{0}$ into itself for each $t \geqslant 0$ then $\mathcal{D}_{0}$ is a core for $A$.

Proposition 2.63. Let $(A, \mathcal{D}(A))$ be the generator of the strongly continuous semigroup $\left(T_{t}\right)_{t \geqslant 0}$ on $\mathcal{B}$ and let $\mathcal{D}_{0}$ be a core for $A$. If $B$ is an operator with domain $\mathcal{D}_{0}$ such that $\left.A\right|_{\mathcal{D}_{0}} \equiv B$, then the closure $\bar{B}$ of $B$ generates the same semigroup $\left(T_{t}\right)_{t \geqslant 0}$, and $\bar{B} \equiv A$. 
Definition 2.64. A. A strongly continuous contractions semigroup $\left(T_{t}\right)_{t \geqslant 0}$ on $C_{\infty}(G)$ is called a Feller semigroup if each operator $T_{t}, t>0$, is positive, that is, if $T_{t} f \geqslant 0$ for any $f \in C_{\infty}(G)$ with $f \geqslant 0$. It is called a strong Feller semigroup if each operator $T_{t}, t>0$, can be extended to an operator which maps $B_{b}(G)$ into $C_{\infty}(G)$.

$B$. An operator $A$ defined on a set $\mathcal{D} \subset C_{\infty}(G)$ is said to satisfy the positive maximum principle on $\mathcal{D}$ if for all $u \in \mathcal{D}$ for which there exists $x_{0} \in G$ such that $u\left(x_{0}\right)=\sup _{x \in G} u(X) \geqslant 0$ follows $A[u]\left(x_{0}\right) \leqslant 0$.

The importance of the positive maximum principle for Feller semigroups is HilleYosida-Ray Theorem, compare [18]:

Theorem 2.65. (Hille-Yosida-Ray Theorem) Let a linear operator $(A, \mathcal{D}(A))$ be on $C_{\infty}(G)$. The operator is closable and its closure of $A$ generates a Feller semigroup on $C_{\infty}(G)$ if and only if the following three conditions hold

1. $\mathcal{D}(A)$ is dense in $\left(C_{\infty}(G),\|\cdot\|_{\infty, G}\right)$;

2. A satisfies the positive maximum principle on $\mathcal{D}(A)$;

3. The range of $(\lambda-A)$ is dense in $\left(C_{\infty}(G),\|\cdot\|_{\infty, G}\right)$ for some $\lambda>0$.

Let $\left(T_{t}\right)_{t \geqslant 0}$ be a $C_{0}$-semigroup on the Banach space $\mathcal{B}$ and let $\left(\mu_{t}\right)_{t \geqslant 0}$ be a vaguely continuous convolution semigroup of sub-probability measures on $[0, \infty)$ with corresponding Bernstein function $f$. Then the Bochner integral

$$
T_{t}^{f} u:=\int_{[0, \infty)} T_{s} u \mu_{t}(d s), t \geqslant 0
$$

defines again a $C_{0}$-contraction semigroup on the Banach space $\mathcal{B}$. We give the definition of subordinate semigroup

Definition 2.66. Let $\left(T_{t}\right)_{t \geqslant 0}$ be a $C_{0}$-semigroup on the Banach space $\mathcal{B}$ and let $\left(\mu_{t}\right)_{t \geqslant 0}$ be a vaguely continuous convolution semigroup of sub-probability measures on $[0, \infty)$ with corresponding Bernstein function $f$. Then the semigroup $\left(T_{t}^{f}\right)_{t \geqslant 0}$ defined by (2.75) is called subordinate semigroup (in the sense of Bochner) to the semigroup $\left(T_{t}\right)_{t \geqslant 0}$ with respect to the Bernstein function $f$. 
Definition 2.67. For $\alpha \in(0,1)$ the family $\left(\sigma_{\alpha}(\cdot, t)\right)_{t>0}$ of density functions on $(0, \infty)$ is defined via the identity

$$
\int_{0}^{\infty} e^{-y z} \sigma_{\alpha}(y, t) d y=e^{-t z^{\alpha}}, \text { Re } z>0
$$

The function $\sigma(\cdot, t), t>0$, are called Lévy stable density functions, see Butzer and Berens [4], and the family $\left(\eta_{t}^{(\alpha)}\right)_{t \geqslant 0}$ of measures, with $\eta_{t}^{(\alpha)}(d y)=\sigma_{\alpha}(y, t) d y$, is called one-sided stable semigroup of order $\alpha$, see Berg and Forst [3]. In particular, for $\alpha=\frac{1}{2}$ there exists an explicit expression for $\sigma_{\alpha}(\cdot, t)$, we have for $y, t>0$

$$
\sigma_{\frac{1}{2}}(y, t)=\frac{1}{\sqrt{2}} y^{-3 / 2} t \exp \left(-\frac{t^{2}}{4 y}\right)
$$

Theorem 2.68. Let $\alpha \in(0,1)$. We have

1. The function $\sigma_{\alpha}(\cdot, t), t>0$, on $(0, \infty)$ are non-negative and integrable with

$$
\int_{0}^{\infty} \sigma_{\alpha}(y, t) d y=1
$$

for all $t>0$.

2. For each $\delta>0$ it is

$$
\lim _{t \mapsto 0} \int_{\delta}^{\infty} \sigma_{\alpha}(y, t) d y=0
$$

3. For $s, t>0$ and $y>0$ it holds

$$
\sigma_{\alpha}(y, s+t)=\int_{0}^{y} \sigma_{\alpha}\left(y-y_{1}, s\right) \sigma_{\alpha}\left(y_{1}, t\right) d y_{1}
$$

Now let $\left(T_{t}\right)_{t \geqslant 0}$ be a strongly continuous contraction semigroup on a Banach space $\mathcal{B}$, and for $\alpha \in(0,1)$ define the operators $T_{t}^{\alpha}$ on $\mathcal{B}$, for $t>0$ and $u \in \mathcal{B}$,

$$
T_{t}^{\alpha} u=\int_{0}^{\infty}\left(T_{s} u\right) \sigma_{\alpha}(s, t) d s,
$$

i.e. as a subordinated semigroup. It can be shown that $\left(T_{t}^{\alpha}\right)_{t \geqslant 0}$ is a strongly continuous contraction semigroup, and its generator $A_{\alpha}$ is the fractional power of the 
generator $A$ of $\left(T_{t}\right)_{T \geqslant 0}$, compare [47], i.e. $A_{\alpha}=-(-A)^{\alpha}$, where the fractional power $(-A)^{\alpha}$ is given by

$$
(-A)^{\alpha} u=\frac{\sin (\alpha \pi)}{\pi} \int_{0}^{\infty} \lambda^{\alpha-1}(\lambda-A)^{-1}(-A u) d \lambda, u \in \mathcal{D}(A),
$$

or

$$
(-A)^{\alpha} u=-\frac{\alpha}{\Gamma(1-\alpha)} \int_{0}^{\infty} \frac{T_{t} u-u}{t^{1+\alpha}} d t, u \in \mathcal{D}(A) .
$$

The resolvent of $A_{\alpha}$ is given by a formula due to Kato [23], [24], see also Yoshida $[47]$,

$$
\left(\mu-A_{\alpha}\right)^{-1} u=\frac{\sin (\alpha \pi)}{\pi} \int_{0}^{\infty} \frac{\lambda^{\alpha}}{\mu^{2}+2 \mu \lambda^{\alpha} \cos (\alpha \pi)+\lambda^{2 \alpha}}(\lambda-A)^{-1} u d \lambda
$$

for $u \in \mathcal{B}$ and $\mu>0$. Compare Komatsu [27] we find the expression

$$
\left(\mu-A_{\alpha}\right)^{-1} u=\int_{0}^{\infty}\left(\frac{\sin (\alpha \pi)}{\pi} \int_{0}^{\infty} e^{-\lambda t} \frac{\lambda^{\alpha}}{\mu^{2}+2 \mu \lambda^{\alpha} \cos (\alpha \pi)+\lambda^{2 \alpha}} d \lambda\right) T_{t} u d t
$$

With the help of the Mittag-Leffler function which is introduced in next chapter, expressions for the resolvent for the Green's functions of the fractional derivatives of order $\alpha \in(0,1)$ are found by Berens and Westphal [2], Gorenflo and Mainardi [7], or Podlubny [34]. These Mittag-Leffler functions are defined for $\mu>0$ and $y \geqslant 0$ by

$$
E_{\alpha, 1}\left(-\mu y^{\alpha}\right)=\sum_{k=0}^{\infty} \frac{\left(-\mu y^{\alpha}\right)^{k}}{\Gamma(\alpha k+1)}
$$

We mention that the functions $e_{\alpha}(\cdot, \mu)$ are differentiable on $(0, \infty)$, and that for their derivatives one has

$$
\frac{E_{\alpha, 1}^{\prime}\left(-\mu y^{\alpha}\right)}{-\mu}=\frac{\sin (\alpha \pi)}{\pi} \int_{0}^{\infty} e^{-y \lambda} \frac{\lambda^{\alpha}}{\mu^{2}+2 \mu \lambda^{\alpha} \cos (\alpha \pi)+\lambda^{2 \alpha}} d \lambda, y>0,
$$

so that $(2.85)$ is

$$
\left(\mu-A_{\alpha}\right)^{-1} u=\int_{0}^{\infty}\left(T_{t} u\right) \frac{E_{\alpha, 1}^{\prime}\left(-\mu t^{\alpha}\right)}{-\mu} d t
$$

Then, we also will need

Theorem 2.69. (Banach's fixed point theorem) Let a operator $A: \mathcal{B} \rightarrow \mathcal{B}$ be a strict contraction on the Banach space $\left(\mathcal{B},\|\cdot\|_{\mathcal{B}}\right)$. Then there exists exactly on fixed point $x_{0}$ of $A$, i.e. $A x_{0}=x_{0}$. Moreover, for every $x_{1} \in \mathcal{B}$ the sequence $\left(A^{k} x_{1}\right)_{k \in \mathbb{N}}$ converges to $x_{0}$. 
The Minkowski's inequality is studied by G.H. Hardy et al. [12]. In our paper, for the case of $L^{p}$ spaces, we need the Minkowski's integral inequality form as, compare E.M. Stein [42],

Theorem 2.70. Minkowski's integral inequality Let $x \in \mathbb{R}^{n}, y \in \mathbb{R}^{m}$ and $F: \mathbb{R}^{n} \times \mathbb{R}^{m} \rightarrow \mathbb{R}$ is measurable. Then for $p>1$ Minkowski's integral inequality is

$$
\left(\int_{\mathbb{R}^{m}}\left|\int_{\mathbb{R}^{n}} F(x, y) d x\right|^{p} d y\right)^{1 / p} \leqslant \int_{\mathbb{R}^{n}}\left(\int_{\mathbb{R}^{m}}|F(x, y)|^{p} d y\right)^{1 / p} d x
$$

where equality holds only if

$$
F(x, y)=\phi(x) \psi(y)
$$

Here we study the Burkholder-Davis-Gundy inequality, compare X.R. Mao [29],

Theorem 2.71. Burkholder-Davis-Gundy inequality Let $f \in L^{2}\left(\mathbb{R}_{+} ; \mathbb{R}^{n \times m}\right)$. Define, for $t \geqslant 0$,

$$
x(t)=\int_{0}^{t} f(s) d B(s)
$$

and

$$
A(t)=\int_{0}^{t}|f(s)|^{2} d s
$$

Then for every $p>0$, there exist universal positive constants $c_{p}, C_{p}$ (only depending on $p)$, such that

$$
c_{p} \mathbb{E}|A(t)|^{p / 2} \leqslant \mathbb{E}\left(\sup _{0 \leqslant s \leqslant t}|x(s)|^{p}\right) \leqslant C_{p} \mathbb{E}|A(t)|^{p / 2},
$$

for all $t \geqslant 0$. In particular, one may take

$$
\begin{aligned}
& c_{p}=\left(\frac{p}{2}\right)^{p}, \quad C_{p}=\left(\frac{32}{p}\right)^{p / 2}, \quad \text { if } 0<p<2 ; \\
& c_{p}=1, \quad C_{p}=4, \quad \text { if } p=2 \text {; } \\
& c_{p}=(2 p)^{-p / 2}, \quad C_{p}=\left[\frac{p^{p+1}}{2(p-1)^{p-1}}\right]^{p / 2}, \quad \text { if } p>2 \text {. }
\end{aligned}
$$




\section{Chapter 3}

\section{Mittag-Leffler Function}

In this chapter, we want to discuss the Mittag-Leffler functions, which play an important roles in various applications of the fractional calculus. More detailed information may be found in the books by A. Erdélyi et al. [6, Vol. 3, Section 18.1], A. Kilbas et al. [26, Section 1.8 to 1.10], F. Mainardi [28, Appendix E] or I. Podlubny [34, Section 1.2].

The one parameter Mittag-Leffler function $E_{\alpha}(z)$ with $\alpha>0$ is defined by the following series representation, convergent in the whole complex plane,

$$
E_{\alpha}(z):=\sum_{n=0}^{\infty} \frac{z^{n}}{\Gamma(\alpha n+1)}, \alpha>0, z \in \mathbb{C},
$$

so $E_{\alpha}(z)$ is an entire function. In the limit for $\alpha \rightarrow 0^{+}$the analyticity in the whole complex plane is lost since

$$
E_{0}(z):=\sum_{n=0}^{\infty} z^{n}=\frac{1}{1-z},|z|<1
$$

In particular, when $\alpha=1$ and $\alpha=2$, we have

$$
E_{1}(z)=e^{z}
$$

and

$$
E_{2}(z)=\cosh (\sqrt{z})
$$


When $\alpha=n, n \in \mathbb{N}$ and $\lambda \in \mathbb{C}$, the following differentiation formulas hold for the function $E_{n}\left(\lambda z^{n}\right)$ :

$$
\left(\frac{d}{d z}\right)^{n} E_{n}\left(\lambda z^{n}\right)=\lambda E_{n}\left(\lambda z^{n}\right)
$$

and

$$
\left(\frac{d}{d z}\right)^{n}\left[z^{n-1} E_{n}\left(\frac{\lambda}{z^{n}}\right)\right]=\frac{(-1)^{n} \lambda}{z^{n+1}} E_{n}\left(\frac{\lambda}{z^{n}}\right), z \neq 0
$$

When $\alpha=1 / n$ and $n \in \mathbb{N} \backslash\{1\}$, the function $E_{1 / n}(z)$ has the following representation:

$$
E_{1 / n}(z)=e^{z^{n}}\left[1+n \int_{0}^{z} e^{-t^{n}}\left(\sum_{k=1}^{n-1} \frac{t^{k-1}}{\Gamma(k / n)}\right) d t\right] .
$$

In particular, for $n=2$, we have

$$
E_{1 / 2}(z)=e^{z^{2}}\left[1+\frac{2}{\sqrt{\pi}} \int_{0}^{z} e^{t^{2}} d t\right]
$$

which yields the asymptotic estimate for $|z| \rightarrow \infty$ and $|\arg (z)|<\frac{\pi}{4}$,

$$
E_{1 / 2}(z) \sim 2 e^{z^{2}}
$$

Before we continue to discuss the asymptotic properties of the Mittag-Leffler function, we want to give an integral representation for $\alpha>0$ and $z \in \mathbb{C}$

$$
E_{\alpha}(z)=\frac{1}{2 \pi i} \int_{H a} \frac{\zeta^{\alpha-1 e^{\zeta}}}{\zeta^{\alpha}-z} d \zeta
$$

where the path of integration $\mathrm{Ha}$ (the Hankel path) is a loop which starts and ends at $-\infty$ and encircles the circular disk $|\zeta| \leqslant|z|^{1 / \alpha}$ in the positive sense: $-\pi \leqslant \arg \zeta \leqslant \pi$ on $\mathrm{Ha}$, as Figure 3.1 .

The integrand in (3.10) has a branch-point at $\zeta=0$. The complex $\zeta$-plane is cut along the negative real axis, and in the cut plane the integrand is single-valued: the principal branch of $\zeta^{\alpha}$ is taken in the cut plane. The integrand has poles at the point $\zeta_{m}=z^{1 / \alpha} e^{2 \pi i m / \alpha}$, where $m$ is an integer, but only those of the poles lie in the cut plane for which $-\alpha \pi<\arg z+2 \pi m<\alpha \pi$. Thus, the number of the poles inside $H a$ is either $[\alpha]$ or $[\alpha+1]$, according to the value of $\arg z$.

Here, we want to study some results taken from [6]. 


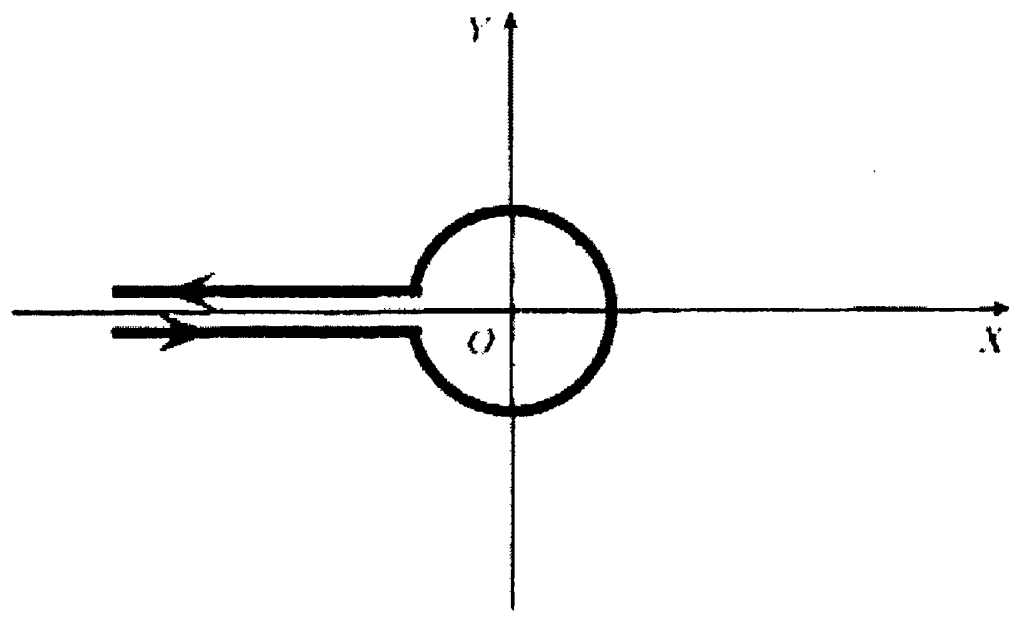

Figure 3.1: The Hankel contour $\mathrm{Ha}$

For the case $0<\alpha<2$ we have for $|z| \rightarrow \infty$,

$$
E_{a}(z) \sim \frac{1}{\alpha} \exp \left(z^{1 / \alpha}\right)-\sum_{k=1}^{\infty} \frac{z^{-k}}{\Gamma(1-\alpha k)},|\arg z|<\alpha \pi / 2
$$

and

$$
E_{a}(z) \sim-\sum_{k=1}^{\infty} \frac{z^{-k}}{\Gamma(1-\alpha k)}, \alpha \pi / 2<\arg z<2 \pi-\alpha \pi / 2
$$

For the case $\alpha \geqslant 2$ we have for $|z| \rightarrow \infty$,

$$
E_{a}(z) \sim \frac{1}{\alpha} \sum_{m} \exp \left(z^{1 / \alpha} e^{2 \pi i m / \alpha}\right) \frac{1}{\alpha} \exp \left(z^{1 / \alpha}\right)-\sum_{k=1}^{\infty} \frac{z^{-k}}{\Gamma(1-\alpha k)}
$$

where $\arg z$ can assume any value between $-\pi$ and $+\pi$ inclusive, and $m$ takes all integer values such that

$$
-\alpha \pi / 2<\arg z+2 \pi m<\alpha \pi / 2
$$

From the asymptotic properties (3.11), (3.12) and (3.13), and the definition of the order of an entire function, we infer that the Mittag-Leffler function is an entire function of order $\rho=1 / \alpha$.

A relevant property of the classical Mittag-Leffler function is its complete monotonicity on the negative real axis, when its parameter $\alpha$ is less or equal 1 . We write 
for $x>0$,

$$
E_{\alpha}(-x) \text { complete monotone iff } 0<\alpha<1
$$

This property, formerly conjectured by Feller using probabilistic methods, was rigorously proven in [35] by $\mathrm{H}$. Polland based on the S. Bochner theorem, which provides a sufficient and necessary condition for the complete monotonicity. In other words, when $0<\alpha<1$ for $x \in \mathbb{R}^{+}$, a representation of $E_{\alpha}(-x)$ is given by

$$
E_{\alpha}(-x)=\int_{0}^{\infty} e^{-u x} P_{\alpha}(u) d u, P_{\alpha}(u) \geqslant 0
$$

Here $P_{\alpha}(u)$ is a transcendent entire function with series representation

$$
P_{\alpha}(u)=\frac{1}{\pi \alpha} \sum_{n=1}^{\infty} \frac{(-1)^{n-1}}{n !} \sin (\pi \alpha n) \Gamma(\alpha n+1) u^{n-1},
$$

whose non-negativity was proven by H. Pollard.

Denoting this inverse Laplace transform by $\mathcal{L}^{-1} P_{\alpha}(u)$, it turns out that

$$
P_{\alpha}(u)=\frac{u^{-1-1 / \alpha}}{\alpha} \mathcal{L}^{-1} P_{\alpha}\left(u^{-1 / \alpha}\right)
$$

with

$$
\mathcal{L}^{-1} P_{\alpha}(u)=-\frac{1}{\pi} \sum_{n=0}^{\infty} \frac{(-1)^{n}}{n !} \sin (\pi \alpha n) \frac{\Gamma(\alpha n+1)}{u^{\alpha n+1}} .
$$

A straightforward generalization of the Mittag-Leffler function is obtained by replacing the additive constant 1 in the argument of the Gamma function in (3.1) by an arbitrary complex parameter $\beta$, i.e. $E_{\alpha, \beta}(z)$. This was first considered by A. Wiman in 1905 , see [36]. thus the function $E_{\alpha, \beta}(z)$ it is defined by

$$
E_{\alpha, \beta}(z):=\sum_{n=0}^{\infty} \frac{z^{n}}{\Gamma(\alpha n+\beta)},
$$

where $\operatorname{Re}(\alpha)>0$ and $\beta, z \in \mathbb{C}$. Of course, for $\beta=1, E_{\alpha, 1}(z) \equiv E_{\alpha}(z)$.

As particular cases, when $\alpha=1$, we have

$$
\begin{gathered}
E_{1,2}(z)=\frac{e^{z}-1}{z} \\
E_{2,2}(z)=\frac{\sinh (\sqrt{z})}{\sqrt{z}}
\end{gathered}
$$




$$
E_{1,3}(z)=\frac{e^{z}-1-z}{z^{2}}
$$

and

$$
E_{1, m}(z)=\frac{1}{z^{m-1}}\left(e^{z}-\sum_{n=0}^{m-2} \frac{z^{n}}{n !}\right) .
$$

When $\alpha=\beta=2$, we have

$$
E_{2,2}\left(+z^{2}\right)=\frac{\sinh (z)}{z}
$$

and

$$
E_{2,2}\left(-z^{2}\right)=\frac{\sin (z)}{z}
$$

Like the Mittag-Leffler function $E_{\alpha}(z), E_{\alpha, \beta}(z)$ is an entire function. We list some general functional relations for the Mittag-Leffler function (3.15) recursive, which involve both the two parameters $\alpha$ and $\beta$, see [6],

$$
E_{\alpha, \beta}(z)=\frac{1}{\Gamma(\beta)}+z E_{\alpha, \alpha+\beta}(z)
$$

and

$$
E_{\alpha, \beta}(z)=\beta E_{\alpha, \beta+1}(z)+\alpha z \frac{d}{d z} E_{\alpha, \beta+1}(z)
$$

Further $E_{\alpha, \beta}$ satisfies the following differentiation formulas for $n \in \mathbb{N}$ and $\lambda \in \mathbb{C}$,

$$
\left(\frac{d}{d z}\right)^{n}\left[z^{\beta-1} E_{\alpha, \beta}\left(\lambda z^{\alpha}\right)\right]=z^{\beta-n-1} E_{\alpha, \beta-n}\left(\lambda z^{\alpha}\right),
$$

and

$$
\left(\frac{d}{d z}\right)^{n}\left[z^{n-\beta} E_{\alpha, \beta}\left(\frac{\lambda}{z^{\alpha}}\right)\right]=\frac{(-1)^{n} \lambda}{z^{\alpha+\beta}} E_{\alpha, \beta}\left(\frac{\lambda}{z^{\alpha}}\right), \text { for } z \neq 0 .
$$

It may be directly proved that the usual derivatives of $E_{\alpha, \beta}(z)$ can be expressed by

$$
\left(\frac{d}{d z}\right)^{n}\left[E_{\alpha, \beta}(z)\right]=n ! E_{\alpha, \beta+\alpha n}^{n+1}(z), \text { for } n \in \mathbb{N}
$$

where $E_{\alpha . \beta}^{n}(z)$ is generalized Mittag-Leffler functions (3.36), see [26, Section 1.9, $\mathrm{p} 45]$. In particular, we have

$$
\left(\frac{d}{d z}\right)^{n}\left[E_{\alpha}(z)\right]=n ! E_{\alpha, 1+\alpha n}^{n+1}(z), \text { for } n \in \mathbb{N} .
$$


When $\alpha=1 / n$ and $n \in \mathbb{N}$, the function $E_{1 / n, \beta}(z)$ has a representation generalizing (3.7):

$$
E_{1 / n, \beta}(z)=z^{(1-\beta) n} e^{z^{n}}\left[z_{0}^{(1-\beta) n} e^{-z_{0}^{n}} E_{1 / n, \beta}\left(z_{0}\right)+n \int_{0}^{z} e^{-t^{n}}\left(\sum_{k=1}^{n} \frac{t^{\beta n-k-1}}{\Gamma(\beta-[k / n])}\right) d t\right]
$$

for any $z_{0} \in \mathbb{C} \backslash\{0\}$.

The two parameter Mittag-Leffler function $E_{\alpha, \beta}(z)$ also has the integral representation for $\alpha, \beta>0$ and $z \in \mathbb{C}$

$$
E_{\alpha, \beta}(z)=\frac{1}{2 \pi i} \int_{H a} \frac{\zeta^{\alpha-\beta} e^{\zeta}}{\zeta^{\alpha}-z} d \zeta
$$

where the path of integration $\mathrm{Ha}$ is the again the Hankel path considered in (3.10) for the one parameter Mittag-Leffler function.

The representation (3.29) can be used to obtain the asymptotic behavior of $E_{\alpha, \beta}(z)$ as $z \rightarrow \infty$. These properties can be summarized as follows.

For the case $0<\alpha<2$ as $|z| \rightarrow \infty$ we have

$$
E_{\alpha, \beta}(z) \sim \frac{1}{\alpha} \exp \left(z^{1 / \alpha}\right)-\sum_{k=1}^{\infty} \frac{z^{-k}}{\Gamma(\beta-\alpha k)},|\arg z|<\alpha \pi / 2
$$

and

$$
E_{\alpha, \beta}(z) \sim-\sum_{k=1}^{\infty} \frac{z^{-k}}{\Gamma(\beta-\alpha k)}, \alpha \pi / 2<\arg z<2 \pi-\alpha \pi / 2
$$

For the case $\alpha \geqslant 2$ as $|z| \rightarrow \infty$ we have

$$
E_{\alpha, \beta}(z) \sim \frac{1}{\alpha} \sum_{m} \exp \left(z^{1 / \alpha} e^{2 \pi i m / \alpha}\right)-\sum_{k=1}^{\infty} \frac{z^{-k}}{\Gamma(\beta-\alpha k)}
$$

where $\arg z$ can assume any value between $-\pi$ and $+\pi$ inclusive and $m$ takes all integer value such that

$$
-\alpha \pi / 2<\arg z+2 \pi m<\alpha \pi / 2
$$

We note that the additional parameter $\beta$ has no influence on the fact of being an entire function, so $E_{\alpha, \beta}(z)$, the two parameters Mittag-Leffler function, is again an entire function. 
Another important property of the two parameters Mittag-Leffler function is the complete monotone on the negative real axis. For $x>0$, we have

$$
E_{\alpha, \beta}(-x) \text { complete monotone iff }\left\{\begin{array}{l}
0<\alpha \leqslant 1 \\
\beta \geqslant \alpha .
\end{array}\right.
$$

We quote that for each $0<\alpha<2$ and $\beta>0$ there exists a constant $C>0$ so that

$$
\left|E_{\alpha, \beta}(z)\right| \leqslant \frac{C}{1+|z|}, \operatorname{Re} z \leqslant 0
$$

Now, we introduce the generalized Mittag-Leffler function defined for $z \in \mathbb{C}$, $\alpha, \beta, \rho \in \mathbb{C}$, and $\Re(\alpha)>0$ by

$$
E_{\alpha, \beta}^{\rho}(z):=\sum_{k=0}^{\infty} \frac{(\rho)_{k} z^{k}}{\Gamma(\alpha k+\beta) k !}
$$

where $(\rho)_{k}$ is the Pochhammer symbol, compare [25] and [37]. In particular, when $\rho=1$, it coincides with the Mittag-Leffler function (3.15), i.e.

$$
E_{\alpha, \beta}^{\rho}(z)=E_{\alpha, \beta}(z)
$$

For the generalized Mittag-Leffler function the following differentiation formulas hold for $n \in \mathbb{N}$ and $\lambda \in \mathbb{C}$

$$
\left(\frac{d}{d z}\right)^{n}\left[E_{\alpha, \beta}^{\rho}(z)\right]=(\rho)_{n} E_{\alpha, \beta+\alpha n}^{\rho+n}(z)
$$

and

$$
\left(\frac{d}{d z}\right)^{n}\left[z^{\beta-1} E_{\alpha, \beta}^{\rho}\left(\lambda z^{\alpha}\right)\right]=z^{\beta-n-1} E_{\alpha, \beta-n}^{\rho}\left(\lambda z^{\alpha}\right) .
$$

The following formula also holds for the Laplace transform of the function $t^{\beta-1} E_{\alpha, \beta}^{\rho}\left(\lambda t^{\alpha}\right)$, we have

$$
\mathcal{L}\left[t^{\beta-1} E_{\alpha, \beta}^{\rho}\left(\lambda t^{\alpha}\right)\right](s)=\frac{s^{\alpha \rho-\beta}}{\left(s^{\alpha}-\lambda\right)^{\rho}}
$$

where $\operatorname{Re} s>0, \operatorname{Re} \beta>0, \lambda \in \mathbb{C}$ and $\left|\lambda s^{\alpha}\right|<1$.

Next, we want to discuss some functions of the Mittag-Leffler type. First, we consider a function defined by

$$
z \mapsto E_{\alpha}\left(\lambda z^{\alpha}\right)
$$


for $z \in \mathbb{C} \backslash\{0\}$ and $\alpha, \lambda \in \mathbb{C}$. The following differentiation formulas hold for this function with respect to $z$ :

$$
\left(\frac{\partial}{\partial z}\right)^{n}\left[E_{\alpha}\left(\lambda z^{\alpha}\right)\right]=z^{-n} E_{\alpha, 1-n}\left(\lambda z^{\alpha}\right)
$$

and with respect to $\lambda$ :

$$
\left(\frac{\partial}{\partial \lambda}\right)^{n}\left[E_{\alpha}\left(\lambda z^{\alpha}\right)\right]=n ! z^{\alpha n} E_{\alpha, \alpha n+1}^{n+1}\left(\lambda z^{\alpha}\right),
$$

for $n \in \mathbb{N}$.

Putting $\rho=\beta=1$ in (3.40), we obtain the Laplace transform of the function (3.41) for $\operatorname{Re} s>0, \lambda \in \mathbb{C}$ and $\left|\lambda s^{-\alpha}\right|<1$

$$
\mathcal{L}\left[E_{\alpha}\left(\lambda t^{\alpha}\right)\right](s)=\frac{s^{\alpha-1}}{s^{\alpha}-\lambda}
$$

Differentiating (3.44) $n$ times with respect $\lambda$ leads to, for $n \in \mathbb{N}$ to

$$
\mathcal{L}\left[t^{\alpha n}\left(\frac{\partial}{\partial \lambda}\right)^{n} E_{\alpha}\left(\lambda t^{\alpha}\right)\right](s)=\frac{n ! s^{\alpha-1}}{\left(s^{\alpha}-\lambda\right)^{n+1}}
$$

Now we consider a more general function defined for $z \in \mathbb{C} \backslash\{0\}, \alpha, \beta, \lambda \in \mathbb{C}$ and $\operatorname{Re} \alpha>0$ by

$$
z^{\beta-1} E_{\alpha, \beta}\left(\lambda z^{\alpha}\right)
$$

The following relations hold for $n \in \mathbb{N}$

$$
\left(\frac{\partial}{\partial z}\right)^{n}\left[z^{\beta-1} E_{\alpha, \beta}\left(\lambda z^{\alpha}\right)\right]=z^{\beta-n-1} E_{\alpha, \beta-n}\left(\lambda z^{\alpha}\right),
$$

and

$$
\left(\frac{\partial}{\partial \lambda}\right)^{n}\left[z^{\beta-1} E_{\alpha, \beta}\left(\lambda z^{\alpha}\right)\right]=n ! z^{\alpha n+\beta-1} E_{\alpha, \alpha n+\beta}^{n+1}\left(\lambda z^{\alpha}\right) .
$$

By using Laplace transform with respect to $t$ for $n \in \mathbb{N}$, Re $s>0, \lambda \in \mathbb{C}$ and $\left|\lambda s^{-\alpha}\right|<1$, we have

$$
\mathcal{L}\left[t^{\beta-1} E_{\alpha, \beta}\left(\lambda t^{\alpha}\right)\right](s)=\frac{s^{\alpha-\beta}}{s^{\alpha}-\lambda}
$$

and

$$
\mathcal{L}\left[t^{\alpha n+\beta-1}\left(\frac{\partial}{\partial \lambda}\right)^{n} E_{\alpha, \beta}\left(\lambda t^{\alpha}\right)\right](s)=\frac{n ! s^{\alpha-\beta}}{\left(s^{\alpha}-\lambda\right)^{n+1}}
$$


Now we consider the special case of the function (3.46) when $\beta=\alpha$. This special function called $\alpha$-Exponential function is defined by

$$
z^{\alpha-1} E_{\alpha, \alpha}\left(\lambda z^{\alpha}\right)
$$

where $z \in \mathbb{C} \backslash\{0\}$, $\operatorname{Re} \alpha>0$ and $\lambda \in \mathbb{C}$. Relations (3.47) to (3.50) yield the corresponding formulas for the function in (3.51) as follows:

$$
\left(\frac{\partial}{\partial z}\right)^{n}\left[z^{\alpha-1} E_{\alpha, \alpha}\left(\lambda z^{\alpha}\right)\right]=z^{\alpha-n-1} E_{\alpha, \alpha-n}\left(\lambda z^{\alpha}\right)
$$

and

$$
\left(\frac{\partial}{\partial \lambda}\right)^{n}\left[z^{\alpha-1} E_{\alpha, \alpha}\left(\lambda z^{\alpha}\right)\right]=n ! z^{(n+1) \alpha-1} E_{\alpha,(n+1) \alpha}^{n+1}\left(\lambda z^{\alpha}\right) .
$$

By using the Laplace transform with respect to $t$ for $n \in \mathbb{N}, \operatorname{Re} s>0, \lambda \in \mathbb{C}$ and $\left|\lambda s^{-\alpha}\right|<1$, we have

$$
\mathcal{L}\left[t^{\alpha-1} E_{\alpha, \alpha}\left(\lambda t^{\alpha}\right)\right](s)=\frac{1}{s^{\alpha}-\lambda}
$$

and

$$
\mathcal{L}\left[\left(\frac{\partial}{\partial \lambda}\right)^{n} E_{\alpha, \alpha}\left(\lambda t^{\alpha}\right)\right](s)=\frac{n !}{\left(s^{\alpha}-\lambda\right)^{n+1}} .
$$

For $\alpha \in(0,1), t>0$ and $x>0$, this special function $E_{\alpha, \alpha}\left(-\lambda t^{\alpha}\right)$ can be written an

$$
E_{\alpha, \alpha}\left(-\lambda t^{\alpha}\right)=\frac{\sin (\alpha \pi)}{\pi} t^{1-\alpha} \int_{0}^{\infty} e^{-x t} \frac{x^{\alpha}}{\left(\lambda+x^{\alpha} \cos (\alpha \pi)\right)^{2}+x^{2 \alpha} \sin (\alpha \pi)} d x
$$




\section{Chapter 4}

\section{Fractional Differential Equation}

In this chapter, we want to investigate the fractional calculus, including the fractional integration and fractional differentiation, compare F. Mainardi [28], I. Podlubny [34] or S. Samko, et al. [38]. The proofs are based on the properties of the two Eulerian integrals, i.e. the Gamma function for $\mathbf{R e} z>0$

$$
\Gamma(z):=\int_{0}^{\infty} e^{-u} u^{z-1} d u
$$

and the Beta function for $\operatorname{Re} p>0, \operatorname{Re} q>0$

$$
\begin{aligned}
B(p, q): & =\int_{0}^{1}(1-u)^{p-1} u^{q-1} d u \\
& =\frac{\Gamma(p) \Gamma(q)}{\Gamma(p+q)} .
\end{aligned}
$$

By noting $\Gamma(n)=(n-1)$ ! and letting $\alpha$ be an arbitrary positive real number, we find the definition of the Riemann-Liouville fractional integral of the order $\alpha>0$ for $f(t)$ :

$$
{ }_{0} I_{t}^{\alpha} f(t):=\frac{1}{\Gamma(\alpha)} \int_{0}^{t}(t-\tau)^{\alpha-1} f(\tau) d \tau, t>0, \alpha \in \mathbb{R}^{+},
$$

where $\mathbb{R}^{+}$is the set of positive real numbers. When $\alpha=0$, we define ${ }_{0} I_{t}^{0}:=I$ (Identity operator), i.e.

$$
{ }_{0} I_{t}^{0} f(t)=f(t) .
$$

Denoting by $\circ$ the composition between operators, we note the semigroup property

$$
{ }_{0} I_{t}^{\alpha} \circ{ }_{0} I_{t}^{\beta}={ }_{0} I_{t}^{\alpha+\beta}, \alpha, \beta \geqslant 0
$$


and their commutative property

$$
{ }_{0} I_{t}^{\alpha} \circ{ }_{0} I_{t}^{\beta}={ }_{0} I_{t}^{\beta} \circ{ }_{0} I_{t}^{\alpha}
$$

We also note the effect of our operators ${ }_{0} I_{t}^{\alpha}$ on the power functions

$$
{ }_{0} I_{t}^{\alpha} t^{\gamma}=\frac{\Gamma(\gamma+1)}{\Gamma(\gamma+1+\alpha)} t^{\gamma+\alpha}
$$

where $\alpha \geqslant 0, \gamma \geqslant-1$ and $t>0$.

After the notion of fractional integral, we shall pay attention on the fractional differentiation. First, we observe that with order $n \in \mathbb{N}$ the local operator of the standard derivative for a given $t, \mathcal{D}_{t}^{n}:=\frac{d^{n}}{d t^{n}}$ is merely the left inverse of the non-local operator of the $n$-fold integral $I_{t}^{n}$, having as a starting point any finite $a<t$. Taking $a \equiv 0$, we define similarly ${ }_{0} \mathcal{D}_{t}^{\alpha}$ as left-inverse to ${ }_{0} I_{t}^{\alpha}$ for $\alpha>0$. Now, let the positive integer $m \in \mathbb{N}$ such that $m-1<\alpha \leqslant m$, and we define the Riemann-Liouville fractional derivative of order $\alpha>0$ :

$$
{ }_{0} \mathcal{D}_{t}^{\alpha} f(t):=\mathcal{D}_{t}^{m} \circ{ }_{0} I^{m-\alpha} f(t), m-1<\alpha \leqslant m,
$$

namely

$$
{ }_{0} \mathcal{D}_{t}^{\alpha} f(t):= \begin{cases}\frac{1}{\Gamma(m-\alpha)} \frac{d^{m}}{d t^{m}} \int_{0}^{t}(t-\tau)^{-\alpha-1+m} f(\tau) d \tau, & m-1<\alpha<m \\ \frac{d^{m}}{d t^{m}} f(t), & \alpha=m,\end{cases}
$$

By using the semigroup property (4.4), we find

$$
{ }_{0} \mathcal{D}_{t}^{\alpha} \circ{ }_{0} I_{t}^{\alpha}=\mathcal{D}_{t}^{m} \circ{ }_{0} I_{t}^{m-\alpha} \circ_{0} I_{t}^{\alpha}=\mathcal{D}_{t}^{m} \circ{ }_{0} I_{t}^{m}=I
$$

Furthermore we obtain

$$
{ }_{0} \mathcal{D}_{t}^{\alpha} t^{\gamma}=\frac{\Gamma(\gamma+1)}{\Gamma(\gamma+1+\alpha)} t^{\gamma+\alpha}
$$

where $\alpha \geqslant 0, \gamma \geqslant-1$ and $t>0$.

In the case $\alpha$ is not integer, the fractional derivative ${ }_{0} \mathcal{D}_{t}^{\alpha} f(t)$ is not zero for the constant function $f(t) \equiv 1$. In fact, (4.9) with $\gamma=0$ gives

$$
{ }_{0} \mathcal{D}_{t}^{\alpha} 1=\frac{t^{-\alpha}}{\Gamma(1-\alpha)}, \text { for } \alpha \geqslant 0, t>0,
$$


which identically vanishes for $\alpha \in \mathbb{N}$, due to the poles of the Gamma function in the points $0,-1,-2, \ldots$

By interchanging in (4.6) the processes of the differentiation and integration we are led to the so-called Caputo fractional derivative (denote by ${ }_{0}^{*} \mathcal{D}_{t}^{\alpha}$ ) of order $\alpha>0$ defined as:

$$
{ }_{0}^{*} \mathcal{D}_{t}^{\alpha}:={ }_{0} I_{t}^{m-\alpha} \circ \mathcal{D}_{t}^{m} f(t), m-1<\alpha<m
$$

namely

$$
{ }_{0}^{*} \mathcal{D}_{t}^{\alpha} f(t):= \begin{cases}\frac{1}{\Gamma(m-\alpha)} \int_{0}^{t}(t-\tau)^{-\alpha-1+m} f^{(m)}(\tau) d \tau, & m-1<\alpha<m, \\ \frac{d^{m}}{d t^{m}} f(t), & \alpha=m,\end{cases}
$$

For non-integer $\alpha$ the definition (4.11) requires the absolute integrability of the derivative of order $m$. Whenever we use the operator ${ }_{0}^{*} \mathcal{D}_{t}^{\alpha}$ we assume that this condition is met.

In fact, assuming that the exchange of the $m$-derivative with the integral is possible, we find the relation between Riemann-Liouville fractional derivative and Caputo fractional derivative:

$$
{ }_{0}^{*} \mathcal{D}_{t}^{\alpha} f(t)={ }_{0} \mathcal{D}_{t}^{\alpha} f(t)-\sum_{k=0}^{m-1} f^{(k)}\left(0^{+}\right) \frac{t^{k-\alpha}}{\Gamma(k-\alpha-1)},
$$

and therefore, recalling the fractional derivative of the power functions (4.9),

$$
{ }_{0}^{*} \mathcal{D}_{t}^{\alpha} f(t)={ }_{0} \mathcal{D}_{t}^{\alpha}\left[f(t)-\sum_{k=0}^{m-1} f^{(k)}\left(0^{+}\right) \frac{t^{k}}{k !}\right] .
$$

In particular for $0<\alpha<1$ we have

$$
{ }_{0}^{*} \mathcal{D}_{t}^{\alpha} f(t)={ }_{0} \mathcal{D}_{t}^{\alpha} f(t)-f\left(0^{+}\right) \frac{t^{-\alpha}}{\Gamma(1-\alpha)}={ }_{0} \mathcal{D}_{0}^{\alpha}\left[f(t)-f\left(0^{+}\right)\right]
$$

In our paper, we shall only focus on the Riemann-Liouville derivative in the future arguments. Let us recall the Laplace transform

$$
\mathcal{L}(f)(z):=\int_{0}^{\infty} e^{-z t} f(t) d t, z \in \mathbb{C}
$$


Corresponding to [28], the Laplace transform for the Riemann-Liouville derivative of order $\alpha$ is given as

$$
\mathcal{L}\left\{{ }_{0} \mathcal{D}_{t}^{\alpha} f(t) ; s\right\}=s^{\alpha} F(s)-\sum_{k=0}^{m-1} s^{m-1-k} g^{(k)}\left(0^{+}\right),
$$

where $g^{(k)}\left(0^{+}\right):=\lim _{t \rightarrow 0^{+}} \mathcal{D}_{t}^{k} g(t)$ and $g(t):={ }_{0} I_{t}^{m-\alpha} f(t)$. The rule (4.16) requires initial values concerning an extra function $g(t)$ related to the given $f(t)$ through a fractional integral. However, when all the limiting values $f^{(k)}\left(0^{+}\right)$for $k=0,1, \ldots$ are finite and the order is not integer, we can obtain that the corresponding $g^{(k)}\left(0^{+}\right)$ vanish so that the formula (4.16) simplifies into

$$
\mathcal{L}\left\{{ }_{0} \mathcal{D}_{t}^{\alpha} f(t) ; s\right\}=s^{\alpha} F(s), m-1<\alpha<m
$$

Note the Laplace transform can be expressed in terms of Mittag-Leffler functions. We already discussed in above chapter. Obviously, we are able to apply the Mittag-Leffler functions to solve to the initial value problem for a non-homogeneous fractional differential equation, see [34]:

$$
{ }_{0} \mathcal{D}_{t}^{\alpha} v(t)+A v(t)=g(t)
$$

and

$$
\left.{ }_{0} \mathcal{D}_{t}^{\alpha-1} v(t)\right|_{t=0}=b
$$

where $0<\alpha<1, t \in[0, T]$ and $T<\infty$.

Let $A, b \in \mathbb{R}$, and let $g(t)$ be a given real function defined on $\mathbb{R}_{+}$. Taking into account the initial condition (4.19), the Laplace transform of (4.18) with respect to $t$ yields

$$
s^{\alpha} V(s)+A V(s)=b+G(s)
$$

or

$$
V(s)=\frac{b}{s^{\alpha}+A}+\frac{G(s)}{s^{\alpha}+A}
$$

By using the inverse Laplace transform, the problem (4.18) and (4.19) give the solution:

$$
v(t)=b t^{\alpha-1} E_{\alpha, \alpha}\left(A t^{\alpha}\right)+\int_{0}^{t}(t-\tau)^{\alpha-1} E_{\alpha, \alpha}\left(A(t-\tau)^{\alpha}\right) g(\tau) d \tau .
$$




\section{Chapter 5}

\section{Estimates for $E_{\alpha, \alpha}\left(-\psi(\xi) t^{\alpha}\right)$}

In this paragraph, we study the function $\xi \rightarrow E_{\alpha, \alpha}\left(-\psi(\xi) t^{\alpha}\right)$. In particular we will discus certain integratility properties depending on properties of $\psi$. In the following we assume that $\psi: \mathbb{R} \rightarrow \mathbb{R}$ is a continuous negative definite function of class $C^{k}, k \geqslant 2$. In this case, compare [14, p145] or [18, p154] we have, compare Theorem 2.40,

$$
\left|\frac{d^{k} \psi(\xi)}{d \xi^{k}}\right| \leqslant \begin{cases}\psi(\xi), & k=0 \\ C_{1} \psi^{1 / 2}(\xi), & k=1 \\ C_{k}, & k \geqslant 2 .\end{cases}
$$

In addition we assume for some $\gamma \in(1,2)$ with $C_{\psi}>0$ that

$$
\psi(\xi) \geqslant C_{\psi}\left(1+|\xi|^{2}\right)^{\gamma / 2}
$$

hold for all $\xi \in \mathbb{R}$.

Lemma 5.1. Suppose that the continuous negative definite function $\psi: \mathbb{R} \rightarrow \mathbb{R}$ satisfies (5.2). For $0<\alpha<1$ it follows that

$$
E_{\alpha, \alpha}\left(-\psi(\cdot) t^{\alpha}\right) \in L^{1}(\mathbb{R}), t>0
$$

and

$$
\left\|E_{\alpha, \alpha}\left(-\psi(\cdot) t^{\alpha}\right)\right\|_{L^{1}} \leqslant C t^{-\alpha} .
$$


Proof. Using (5.2) we find for $t>0$

$$
\frac{1}{1+t^{\alpha} \psi(\xi)} \leqslant \frac{1}{1+t^{\alpha} C_{\psi}\left(1+|\xi|^{2}\right)^{\gamma / 2}}
$$

Now by (3.35) we get

$$
\begin{aligned}
& \int_{\mathbb{R}}\left|E_{\alpha, \alpha}\left(-\psi(\xi) t^{\alpha}\right)\right| d \xi \\
& \leqslant \int_{\mathbb{R}} \frac{C_{\alpha, \alpha}}{1+\psi(\xi) t^{\alpha}} d \xi \\
& \leqslant \int_{\mathbb{R}} \frac{C_{\alpha, \alpha}}{1+t^{\alpha} C_{\psi}\left(1+|\xi|^{2}\right)^{\gamma / 2}} d \xi \\
& \leqslant \frac{C_{\alpha, \alpha}}{C_{\psi} t^{\alpha}} \int_{\mathbb{R}} \frac{1}{\left(1+|\xi|^{\gamma / 2}\right)} d \xi,
\end{aligned}
$$

and since $\gamma>1$ it follows $E_{\alpha, \alpha}\left(-\psi(\cdot) t^{\alpha}\right) \in L^{1}(\mathbb{R})$ and (5.4) holds.

Lemma 5.2. Suppose that the continuous negative definite function $\psi: \mathbb{R} \rightarrow \mathbb{R}$ satisfies (5.1) and (5.2). For $0<\alpha<1$ it follows that

$$
\left|\frac{d^{2}}{d \xi^{2}} E_{\alpha, \alpha}\left(-\psi(\xi) t^{\alpha}\right)\right| \leqslant \tilde{C}_{\alpha, \psi} \frac{1}{\left(1+|\xi|^{2}\right)^{\gamma / 2}} \frac{1}{\left(1+t^{\alpha}\right)}
$$

Proof. Using (3.35) we find

$$
\begin{aligned}
& \frac{d}{d \xi} E_{\alpha, \alpha}\left(-\psi(\xi) t^{\alpha}\right) \\
& =\left(-\psi^{\prime}(\xi) t^{\alpha}\right)\left(E_{\alpha, \alpha}^{\prime}\right)\left(-\psi(\xi) t^{\alpha}\right) \\
& =\left(-\psi^{\prime}(\xi) t^{\alpha}\right)\left(\frac{1}{-\alpha \psi(\xi) t^{\alpha}} E_{\alpha, \alpha-1}\left(-\psi(\xi) t^{\alpha}\right)-\frac{\alpha-1}{-\alpha \psi(\xi) t^{\alpha}} E_{\alpha, \alpha}\left(-\psi(\xi) t^{\alpha}\right)\right) \\
& =\frac{\psi^{\prime}(\xi)}{\alpha \psi(\xi)}\left(E_{\alpha, \alpha-1}\left(-\psi(\xi) t^{\alpha}\right)-(1-\alpha) E_{\alpha, \alpha}\left(-\psi(\xi) t^{\alpha}\right)\right)
\end{aligned}
$$


and

$$
\begin{aligned}
& \frac{d^{2}}{d \xi^{2}} E_{\alpha, \alpha}\left(-\psi(\xi) t^{\alpha}\right) \\
&=\frac{d}{d \xi}\left(\frac{d}{d \xi} E_{\alpha, \alpha}\left(-\psi(\xi) t^{\alpha}\right)\right) \\
&=\frac{d}{d \xi}\left(\frac{\psi^{\prime}(\xi)}{\alpha \psi(\xi)}\left(E_{\alpha, \alpha-1}\left(-\psi(\xi) t^{\alpha}\right)-(1-\alpha) E_{\alpha, \alpha}\left(-\psi(\xi) t^{\alpha}\right)\right)\right. \\
&=\left(\frac{d}{d \xi} \frac{\psi^{\prime}(\xi)}{\alpha \psi(\xi)}\right)\left(E_{\alpha, \alpha-1}\left(-\psi(\xi) t^{\alpha}\right)+(1-\alpha) E_{\alpha, \alpha}\left(-\psi(\xi) t^{\alpha}\right)\right) \\
& \quad+\frac{\psi^{\prime}(\xi)}{\alpha \psi(\xi)}\left(\frac{d}{d \xi} E_{\alpha, \alpha-1}\left(-\psi(\xi) t^{\alpha}\right)+(1-\alpha) E_{\alpha, \alpha}\left(-\psi(\xi) t^{\alpha}\right)\right) \\
&=\left(\frac{\psi^{\prime \prime}(\xi) \psi(\xi)-\psi^{\prime}(\xi)^{2}}{\alpha \psi(\xi)^{2}}\right)\left(E_{\alpha, \alpha-1}\left(-\psi(\xi) t^{\alpha}\right)+(1-\alpha) E_{\alpha, \alpha}\left(-\psi(\xi) t^{\alpha}\right)\right) \\
&+\left(\frac{\psi^{\prime}(\xi)}{\alpha \psi(\xi)}\right)\left(\left(-\psi^{\prime}(\xi) t^{\alpha}\right)\left(\left(E_{\alpha, \alpha-1}^{\prime}\right)\left(-\psi(\xi) t^{\alpha}\right)+(1-\alpha)\left(E_{\alpha, \alpha}^{\prime}\right)\left(-\psi(\xi) t^{\alpha}\right)\right)\right) \\
&=\left(\frac{\psi^{\prime \prime}(\xi) \psi(\xi)-\psi^{\prime}(\xi)^{2}}{\alpha \psi(\xi)^{2}}\right)\left(E_{\alpha, \alpha-1}\left(-\psi(\xi) t^{\alpha}\right)+(1-\alpha) E_{\alpha, \alpha}\left(-\psi(\xi) t^{\alpha}\right)\right) \\
&+\left(\frac{\psi^{\prime}(\xi)}{\alpha \psi(\xi)}\right)^{2}\left(E_{\alpha, \alpha-2}\left(-\psi(\xi) t^{\alpha}\right)\right. \\
&\left.+2(1-\alpha) E_{\alpha, \alpha-1}\left(-\psi(\xi) t^{\alpha}\right)-(1-\alpha)^{2} E_{\alpha, \alpha}\left(-\psi(\xi) t^{\alpha}\right)\right)
\end{aligned}
$$

i.e. we have

$$
\begin{aligned}
& \frac{d^{2}}{d \xi^{2}} E_{\alpha, \alpha}\left(-\psi(\xi) t^{\alpha}\right) \\
& =\left(\frac{\psi^{\prime \prime}(\xi) \psi(\xi)-\psi^{\prime}(\xi)^{2}}{\alpha \psi(\xi)^{2}}\right)\left(E_{\alpha, \alpha-1}\left(-\psi(\xi) t^{\alpha}\right)+(1-\alpha) E_{\alpha, \alpha}\left(-\psi(\xi) t^{\alpha}\right)\right) \\
& +\left(\frac{\psi^{\prime}(\xi)}{\alpha \psi(\xi)}\right)^{2}\left(E_{\alpha, \alpha-2}\left(-\psi(\xi) t^{\alpha}\right)\right. \\
& \left.\quad+2(1-\alpha) E_{\alpha, \alpha-1}\left(-\psi(\xi) t^{\alpha}\right)-(1-\alpha)^{2} E_{\alpha, \alpha}\left(-\psi(\xi) t^{\alpha}\right)\right)
\end{aligned}
$$


Since $\left|E_{\alpha, \beta}(z)\right| \leqslant \frac{C_{\alpha, \beta}}{1+|z|}$ for $\operatorname{Re} z \leqslant 0, \alpha \in(0,1)$ and $\beta \in \mathbb{R}$, we find using (5.2) that

$$
\begin{aligned}
& \left|\frac{d^{2}}{d \xi^{2}} E_{\alpha, \alpha}\left(-\psi(\xi) t^{\alpha}\right)\right| \\
& \leqslant\left|\left(\frac{\psi^{\prime}(\xi)}{\alpha \psi(\xi)}\right)^{2} E_{\alpha, \alpha-2}\left(-\psi(\xi) t^{\alpha}\right)\right| \\
& +\left|\left(\frac{\alpha \psi^{\prime \prime}(\xi) \psi(\xi)+(2-3 \alpha) \psi^{\prime}(\xi)^{2}}{\alpha^{2} \psi(\xi)^{2}}\right) E_{\alpha, \alpha-1}\left(-\psi(\xi) t^{\alpha}\right)\right| \\
& +\left|\left(\frac{\left(\alpha-\alpha^{2}\right) \psi^{\prime \prime}(\xi) \psi(\xi)+(\alpha-1) \psi^{\prime}(\xi)^{2}}{\alpha^{2} \psi(\xi)^{2}}\right) E_{\alpha, \alpha}\left(-\psi(\xi) t^{\alpha}\right)\right| \\
& \leqslant C_{\alpha, \psi} \frac{1}{\psi(\xi)}\left(\frac{1}{1+\psi(\xi) t^{\alpha}}\right) \\
& \leqslant \tilde{C}_{\alpha, \psi} \frac{1}{\left(1+|\xi|^{2}\right)^{\gamma / 2}} \frac{1}{\left(1+t^{\alpha}\right)} \text {. }
\end{aligned}
$$

which proves the lemma.

Remark 5.3. The proof of Lemma 5.2 also yields the estimate

$$
\left|\frac{d}{d \xi} E_{\alpha, \alpha}\left(-\psi(\xi) t^{\alpha}\right)\right| \leqslant C_{\alpha, \psi}^{*} \frac{1}{\left(1+|\xi|^{2}\right)^{\gamma / 4}} \frac{1}{1+t^{\alpha}} .
$$

Corollary 5.4. Suppose that $\psi$ satisfies (5.2). Then for all $t>0$ we have

$$
E_{\alpha, \alpha}\left(-\psi(\cdot) t^{\alpha}\right) \in L^{1}(\mathbb{R}) \cap L^{\infty}(\mathbb{R})
$$

in particular $E_{\alpha, \alpha}\left(-\psi(\cdot) t^{\alpha}\right) \in L^{p}(\mathbb{R})$ for all $1 \leqslant p \leqslant \infty$.

Proof. By Lemma 5.1 we know that $E_{\alpha, \alpha}\left(-\psi(\cdot) t^{\alpha}\right) \in L^{1}(\mathbb{R})$ for $t>0$. Moreover, by (3.35) we have

$$
\left|E_{\alpha, \alpha}\left(-\psi(\xi) t^{\alpha}\right)\right| \leqslant \frac{C_{\alpha, \alpha}}{1+\psi(\xi) t^{\alpha}} \leqslant C_{\alpha, \alpha}
$$

which yields $E_{\alpha, \alpha}\left(-\psi(\cdot) t^{\alpha}\right) \in L^{\infty}(\mathbb{R})$.

Corollary 5.5. Let $f \in L^{p}(\mathbb{R})$ for some $p, 1 \leqslant p \leqslant \infty$. Then $f \cdot E_{\alpha, \alpha}\left(-\psi(\cdot) t^{\alpha}\right) \in$ $L^{1}(\mathbb{R})$ for $t>0$, i.e. the integral

$$
\int_{\mathbb{R}} f(\xi) E_{\alpha, \alpha}\left(-\psi(\xi) t^{\alpha}\right) d \xi
$$

is for every $t>0$ finite. 
Since under assumption (5.2) we know $E_{\alpha, \alpha}\left(-\psi(\cdot) t^{\alpha}\right) \in L^{1}(\mathbb{R})$ the inverse Fourier transform $F^{-1}\left(E_{\alpha, \alpha}\left(-\psi(\cdot)(t-\tau)^{\alpha}\right)\right)$ is well defined and belongs to $C_{\infty}(\mathbb{R})$ for $t, \tau$, $t>\tau$, being fixed. We claim

Lemma 5.6. If the continuous negative definite function $\psi: \mathbb{R} \rightarrow \mathbb{R}$ satisfies (5.2) and is of class $C^{2}$ then $F^{-1}\left(E_{\alpha, \alpha}\left(-\psi(\cdot)(t-\tau)^{\alpha}\right)\right)$ belongs for $0<\tau<t$ to $L^{1}(\mathbb{R})$ and it holds

$$
h(t, \tau):=\left\|F^{-1}\left(E_{\alpha, \alpha}\left(-\psi(\cdot)(t-\tau)^{\alpha}\right)\right)\right\|_{L^{1}} \leqslant \kappa_{1}(t-\tau)^{-\alpha / \gamma}+\kappa_{2}\left(1+(t-\tau)^{\alpha}\right)^{-1} .
$$

Proof. Since $\psi$ is even we find with $t-\tau=s$ that

$$
\begin{aligned}
& \left\|F^{-1}\left(E_{\alpha, \alpha}\left(-\psi(\cdot) s^{\alpha}\right)\right)\right\|_{L^{1}} \\
& =(2 \pi)^{-1 / 2} \int_{\mathbb{R}}\left|\int_{\mathbb{R}} e^{i x \xi} E_{\alpha, \alpha}\left(-\psi(\xi) s^{\alpha}\right) d \xi\right| d x \\
& =(2 \pi)^{-1 / 2} \int_{-1}^{1}\left|\int_{\mathbb{R}} e^{i x \xi} E_{\alpha, \alpha}\left(-\psi(\xi) s^{\alpha}\right) d \xi\right| d x \\
& \quad+(2 \pi)^{-1 / 2} \int_{1}^{\infty}\left|\int_{\mathbb{R}} e^{i x \xi} E_{\alpha, \alpha}\left(-\psi(\xi) s^{\alpha}\right) d \xi\right| d x \\
& \quad+(2 \pi)^{-1 / 2} \int_{-\infty}^{-1}\left|\int_{\mathbb{R}} e^{i x \xi} E_{\alpha, \alpha}\left(-\psi(\xi) s^{\alpha}\right) d \xi\right| d x \\
& \leqslant(2 \pi)^{-1 / 2} \int_{-1}^{1}\left(\int_{\mathbb{R}} \frac{C}{1+s^{\alpha} C_{0}\left(1+|\xi|^{2}\right)^{\gamma / 2}} d \xi\right) d x \\
& \quad+(2 \pi)^{-1 / 2} \int_{1}^{\infty}\left|\int_{\mathbb{R}} \frac{1}{x^{2}}\left(\frac{d^{2}}{d \xi^{2}} e^{i x \xi}\right) E_{\alpha, \alpha}\left(-\psi(\xi) s^{\alpha}\right) d \xi\right| d x \\
& \quad+(2 \pi)^{-1 / 2} \int_{-\infty}^{-1}\left|\int_{\mathbb{R}} \frac{1}{x^{2}}\left(\frac{d^{2}}{d \xi^{2}} e^{i x \xi}\right) E_{\alpha, \alpha}\left(-\psi(\xi) s^{\alpha}\right) d \xi\right| d x
\end{aligned}
$$

For the first integral we find

$$
\begin{aligned}
& (2 \pi)^{-1 / 2} \int_{-1}^{1}\left(\int_{\mathbb{R}} \frac{C}{1+s^{\alpha} C_{0}\left(1+|\xi|^{2}\right)^{\gamma / 2}} d \xi\right) d x \\
& \leqslant(2 \pi)^{-1 / 2} \int_{-1}^{1} \int_{\mathbb{R}} \frac{C}{1+s^{\alpha} C_{0}|\xi|^{\gamma}} d \xi d x \\
& =\frac{2(2 \pi)^{-1 / 2} C}{\gamma C_{0}^{1 / \gamma}} B\left(\frac{1}{\gamma}, 1-\frac{1}{\gamma}\right) t^{-\alpha / \gamma} \\
& =\kappa_{1} t^{-\alpha / \gamma}
\end{aligned}
$$


where $B(\alpha, \beta)$ denotes the Euler Beta-function.

Integrating in the inner integrals of the second and third term by parts and taking the decay of $E_{\alpha, \alpha}\left(-\psi(\xi) t^{\alpha}\right)$ into account, we find using (5.7)

$$
\begin{aligned}
& (2 \pi)^{-1 / 2} \int_{1}^{\infty}\left|\int_{\mathbb{R}} \frac{1}{x^{2}}\left(\frac{d^{2}}{d \xi^{2}} e^{i x \xi}\right) E_{\alpha, \alpha}\left(-\psi(\xi) s^{\alpha}\right) d \xi\right| d x \\
& \quad+(2 \pi)^{-1 / 2} \int_{-\infty}^{-1}\left|\int_{\mathbb{R}} \frac{1}{x^{2}}\left(\frac{d^{2}}{d \xi^{2}} e^{i x \xi}\right) E_{\alpha, \alpha}\left(-\psi(\xi) s^{\alpha}\right) d \xi\right| d x \\
& \leqslant \tilde{C}_{\alpha} \int_{1}^{\infty} \int_{\mathbb{R}}\left(\frac{1}{x^{2}} \frac{1}{\left(1+|\xi|^{2}\right)^{\gamma / 2}} \frac{1}{1+s^{\alpha}}\right) d \xi d x \\
& =\frac{\tilde{C}_{\alpha}}{2\left(1+s^{\alpha}\right)} B\left(\frac{1}{2}, \frac{\gamma-1}{2}\right) \\
& =\kappa_{2}\left(1+s^{\alpha}\right)^{-1}
\end{aligned}
$$

which yields eventually

$$
h(t, \tau) \leqslant \kappa_{1}(t-\tau)^{-\alpha / \gamma}+\kappa_{2}\left(1+(t-\tau)^{\alpha}\right)^{-1}
$$

for $0<\tau<t$.

Corollary 5.7. Let $\psi$ be as in Lemma 5.6. For $\gamma>1$ and $\alpha>0$ the function

$$
t \mapsto \tilde{h}(t):=t^{\alpha-1}\left\|F^{-1}\left(E_{\alpha, \alpha}\left(-\psi(\cdot) t^{\alpha}\right)\right)\right\|_{L^{1}}
$$

is integrable on every finite interval $[0, T]$ and we have

$$
\int_{0}^{T} \tilde{h}(\tau) d \tau \leqslant \frac{\kappa_{1}}{\alpha(\gamma-1)} T^{\alpha(\gamma-1) / \gamma}+\frac{\kappa_{2}}{\alpha} T^{\alpha} .
$$

Proof. Using Lemma 5.6 we deduce

$$
\tilde{h}(t) \leqslant \kappa_{1} t^{\alpha-1-\frac{\alpha}{\gamma}}+\kappa_{2} \frac{t^{\alpha-1}}{1+t^{\alpha}}
$$

and since $\alpha-1-\frac{\alpha}{\gamma}=\frac{\alpha}{\gamma}(\gamma-1)-1>-1$ and $\alpha-1>-1$ the integrability follows. Moreover we have

$$
\int_{0}^{T} \tilde{h}(s) d s \leqslant \frac{\kappa_{1}}{\alpha(\gamma-1)} T^{\alpha(\gamma-1) / \gamma}+\frac{\kappa_{2}}{\alpha} T^{\alpha} .
$$


Note that (5.14) implies

$$
\sup _{0 \leqslant t \leqslant T} \int_{0}^{t}(t-\tau)^{\alpha-1} h(t, \tau) d \tau \leqslant \frac{\kappa_{1}}{\alpha(\gamma-1)} T^{\frac{\alpha}{\gamma}(\gamma-1)}+\frac{\kappa_{2}}{\alpha} T^{\alpha}
$$

and in particular we have

$$
\lim _{T \rightarrow 0}\left(\sup _{0 \leqslant t \leqslant T} \int_{0}^{t}(t-\tau)^{\alpha-1} h(t, \tau) d \tau\right)=0
$$

which yields that for every $d>0$ we can find $T_{0}$ such that

$$
d \sup _{0 \leqslant t \leqslant T_{0}} \int_{0}^{t}(t-\tau)^{\alpha-1} h(t, \tau) d \tau \leqslant \kappa<1 .
$$




\section{Chapter 6}

\section{Pseudo-differential Operator with}

\section{Negative Definite Symbols and}

\section{Calderón-Vaillancourt Theorem}

In this chapter, we want to investigate pseudo-differential operators with negative definite symbols and then we prove the Calderó-Vaillancourt theorem. The class of pseudo-differential operators is determined by Courrège's results, compare [18], which characterizes generators of Feller processes

$$
q(x, D) u(x)=(2 \pi)^{-n / 2} \int_{\mathbb{R}^{n}} e^{i x \cdot \xi} q(x, \xi) \hat{u}(\xi) d \xi
$$

where we assume that $q: \mathbb{R}^{n} \times \mathbb{R}^{n} \rightarrow \mathbb{C}$ is a locally bounded function such that for every $x \in \mathbb{R}^{n}$ the function $q(x, \cdot): \mathbb{R}^{n} \rightarrow \mathbb{C}$ is a negative definite and continuous.

Before studying the pseudo-differential operator, we want to introduce briefly a family of anisotropic Sobolev spaces $H^{\psi, s}\left(\mathbb{R}^{n}\right)$ of functions on $\mathbb{R}^{n}$ associated to the function $\psi$. We denote the classical Sobolev spaces of order $s \geqslant 0$ with

$$
H^{s}\left(\mathbb{R}^{n}\right)=\left\{u \in L^{2}\left(\mathbb{R}^{n}\right):\|u\|_{H^{s}\left(\mathbb{R}^{n}\right)}<\infty\right\}
$$

where

$$
\|u\|_{H^{s}\left(\mathbb{R}^{n}\right)}=\left(\int_{\mathbb{R}^{n}}\left(1+|\xi|^{2}|\hat{u}(\xi)|^{2} d \xi\right)\right)^{1 / 2}
$$


and $\hat{u}$ denotes the Fourier transform of the function $u$.

Definition 6.1. For $s \geqslant 0$ define the space $H^{\psi, s}\left(\mathbb{R}^{n}\right)$ of all functions $u \in L^{2}\left(\mathbb{R}^{n}\right)$ with finite norm

$$
\|u\|_{\mathbb{R}^{n}, \psi, s}:=\left(\int_{\mathbb{R}^{n}}(1+\psi(\xi))^{2 s}|\hat{u}(\xi)|^{2} d \xi\right)^{1 / 2}
$$

The spaces $H^{\psi, s}\left(\mathbb{R}^{n}\right)$ are Hilbert spaces with inner product $\langle\cdot, \cdot\rangle_{\mathbb{R}^{n}, \psi, s}$ given by

$$
\langle u, v\rangle_{\mathbb{R}^{n}, \psi, s}=\int_{\mathbb{R}^{n}}(1+\psi(\xi))^{2 s} \hat{u}(\xi) \overline{\hat{v}(\xi)} d \xi, u, v \in H^{\psi, s}\left(\mathbb{R}^{n}\right)
$$

and by the Plancherel theorem (see [40]) it is $H^{\psi, 0}\left(\mathbb{R}^{n}\right) \simeq L^{2}\left(\mathbb{R}^{n}\right)$, and for $s>t \geqslant 0$ we have $H^{\psi, s}\left(\mathbb{R}^{n}\right) \subset H^{\psi, t}\left(\mathbb{R}^{n}\right)$ with $\|u\|_{\mathbb{R}^{n}, \psi, t} \leqslant\|u\|_{\mathbb{R}^{n}, \psi, s}$ for all $u \in H^{\psi, s}\left(\mathbb{R}^{n}\right)$.

Proposition 6.2. For each $s \geqslant 0$ the space $C_{0}^{\infty}\left(\mathbb{R}^{n}\right)$ is dense in $\left(H^{\psi, s}\left(\mathbb{R}^{n}\right),\|\cdot\|_{\psi, s}\right)$.

Let $\alpha \in(0,1)$ and $\psi: \mathbb{R}^{n} \rightarrow \mathbb{R}$ be a real-valued, non-negative, continuous and negative definite function on $\mathbb{R}^{n}$. We suppose that the function $\psi$ has the property (P.1), i.e.

(P.1)There exists $r_{1}>0$ such that for all $s>r_{1}$

$$
\int_{\mathbb{R}^{n}} \frac{1}{(1+\psi(\xi))^{2 s}} d s<\infty
$$

Proposition 6.3. Suppose that the function $\psi$ has the property (P.1) (6.6), and let $s>r_{1}$. Then $H^{\psi, s}\left(\mathbb{R}^{n}\right)$ can be identified with a subspace of $C_{\infty}\left(\mathbb{R}^{n}\right)$, and in this sense we write $H^{\psi, s}\left(\mathbb{R}^{n}\right) \subset C_{\infty}\left(\mathbb{R}^{n}\right)$. There exists a constant $c_{s}>0$, depending on $s$ and $\psi$, such that for all $u \in H^{\psi, s}\left(\mathbb{R}^{n}\right)$ holds

$$
\|u\|_{\infty, \mathbb{R}^{n}} \leqslant c_{s}\|u\|_{\psi, s}
$$

Corollary 6.4. Let $\psi$ satisfy the property (P.1) (6.6), and let $s>r_{1}$. The space $H^{\psi, s}\left(\mathbb{R}^{n}\right)$ is dense in $\left(C_{\infty}\left(\mathbb{R}^{n}\right),\|\cdot\|_{\infty, \mathbb{R}^{n}}\right)$. 
Now let us return to a pseudo-differential operator with continuous symbol $q$ : $\mathbb{R}^{n} \times \mathbb{R}^{n} \mapsto \mathbb{R}$ such that for each $x \in \mathbb{R}^{n}$ the function $q(x, \cdot): \mathbb{R}^{n} \rightarrow \mathbb{R}$ is negative definite.

Theorem 6.5. Let $q: \mathbb{R}^{n} \times \mathbb{R}^{n} \rightarrow \mathbb{R}$ be given as above, and assume that there exists a continuous negative definite function $\psi: \mathbb{R}^{n} \rightarrow \mathbb{R}$ with the property (P.1) (6.6), and a constant $c_{s}>0$, for $s>r_{1}$, such that

$$
\|q(x, D) u\|_{\mathbb{R}^{n}, \psi, s} \leqslant c_{s}\|u\|_{\mathbb{R}^{n}, \psi, s+2}
$$

for all $u \in C_{0}^{\infty}\left(\mathbb{R}^{n}\right)$. Then $-q(x, D)$ can be extended to an operator on $C_{\infty}\left(\mathbb{R}^{n}\right)$ with domain $H^{\psi, s+2}\left(\mathbb{R}^{n}\right) \subset C_{\infty}\left(\mathbb{R}^{n}\right)$, and this extension satisfies the positive maximum principle on $H^{\psi, s+2}\left(\mathbb{R}^{n}\right)$.

We want to quote two results on the existence of Feller semigroup generated by pseudo-differential operators with negative definite symbol. The first result is due to W. Hoh [14], compare also [19],

Theorem 6.6. Let $q: \mathbb{R}^{n} \times \mathbb{R}^{n} \rightarrow \mathbb{R}$ be a continuous function such that for each $x \in \mathbb{R}^{n}$ the function $q(x, \cdot): \mathbb{R}^{n} \rightarrow \mathbb{R}$ is negative definite. Furthermore assume that $\psi: \mathbb{R}^{n} \rightarrow \mathbb{R}$ is a continuous negative definite function which satisfies $(1+\psi(\xi)) \geqslant c\left(1+|\xi|^{2}\right)^{\gamma / 2}$ for some $0<\gamma \leqslant 2$ and all $\xi \in \mathbb{R}$. Let $M:=\min \left\{m \in \mathbb{N}: m>\max \left(\frac{n}{\gamma}, 2\right)+n\right\}$ and $k:=2 M+1-n$. The operator $\left(-q(x, D), C_{0}^{\infty}\left(\mathbb{R}^{n}\right)\right)$ can be extended to a generator of a Feller semigroup if the following three conditions hold

1. For each $\xi \in \mathbb{R}^{n}$ the map $x \mapsto q(x, \xi)$ is in $C^{k}\left(\mathbb{R}^{n}\right)$ and for all $(x, \xi) \in \mathbb{R}^{n} \times \mathbb{R}^{n}$ and $\beta \in \mathbb{N}_{0}^{n}$ with $|\beta| \leqslant k$ holds $\left|\partial_{x}^{\beta} q(x, \xi)\right| \leqslant c(1+\psi(\xi))$.

2. There exist $\lambda: \mathbb{R}^{n} \rightarrow(0, \infty)$ and $\varrho>0$ such that for $|\xi|>\varrho$ and $x \in \mathbb{R}^{n}$ it is $q(x, \xi) \geqslant \lambda(x) \psi(\xi)$. 
3. There exists a continuous function $\gamma: \mathbb{R}^{n} \rightarrow[0, \infty)$ with $\gamma(0)=0$ so that for $|\xi| \leqslant \varrho$ and $x \in \mathbb{R}^{n}$ hold $q(x, \xi) \leqslant \gamma(\xi)$.

The following result is due to N. Jacob [16], compare also [19]. Suppose that the symbol $q$ is decomposed into two functions $q_{1}: \mathbb{R}^{n} \rightarrow \mathbb{R}$ and $q_{2}: \mathbb{R}^{n} \times \mathbb{R}^{n} \rightarrow \mathbb{R}$, i.e. $q(x, \xi)=q_{1}(\xi)+q_{2}(x, \xi)$, which can always be made for instance by fixing $x_{0} \in \mathbb{R}^{n}$ and setting $q(x, \xi)=q\left(x_{0}, \xi\right)+\left(q(x, \xi)-q\left(x_{0}, \xi\right)\right)$. Given the negative definite function $\psi: \mathbb{R}^{n} \rightarrow \mathbb{R}$ we say that $q_{1}$ has property (P.2) if the condition hold (P.2) There exist constants $0<\gamma_{1} \leqslant \gamma_{2}$ and $\varrho>0$ such that $\left|q_{1}(\xi)\right| \leqslant \gamma_{1}(1+\psi(\xi))$ for all $\xi \in \mathbb{R}^{n}$, and $q_{1}(\xi) \geqslant \gamma_{2} \psi(\xi)$ for all $\xi \in \mathbb{R}^{n}$ with $|\xi| \geqslant \varrho$.

Definition 6.7. For $s, t>0$ let

$$
\tau_{\psi}(s, t):=\int_{\mathbb{R}^{n}} \frac{1+\psi(\xi)^{s}}{\left(1+|\xi|^{2}\right)^{t / 2}} d \xi
$$

and for $s>0$ define $q_{\psi}(s):=\min \left\{q \in \mathbb{N}: \tau_{\psi}(s, q)<\infty\right\}$.

If the Fourier transforms $F_{\mathbb{R}^{n}}\left[q_{2}(\cdot, \eta)\right]$ in the first variable of the function $q_{2}$ exist for all $\eta \in \mathbb{R}^{n}$, then we define

$$
\hat{q}_{2}(\xi, \eta):=(2 \pi)^{-n / 2} \int_{\mathbb{R}^{n}} e^{-i x \xi} q_{2}(x, \eta) d x, \xi \in \mathbb{R}^{n},
$$

and in terms of $\hat{q}_{2}$ we formulate property (P.3), $s \geqslant 0$, as follows:

(P.3) For each $N \in \mathbb{N}$ with $0 \leqslant N \leqslant q_{\psi}(s)$ there exists a constant $\tilde{\gamma}(N)>0$ such that for all $\xi, \eta \in \mathbb{R}^{n}$ holds, compare [16],

$$
\left|q_{2}(\xi, \eta)\right| \leqslant \tilde{\gamma}(N)\left(1+|\xi|^{2}\right)^{-N / 2}(1+\psi(\eta))
$$

Lemma 6.8. Let $\psi: \mathbb{R}^{n} \rightarrow \mathbb{R}$ be negative definite, $s \geqslant 0$ and $q_{2}: \mathbb{R}^{n} \times \mathbb{R}^{n} \rightarrow \mathbb{R}$. If $q_{2}(\cdot, \xi)$ is $q_{\psi}(s)$-times continuously differentiable for all $\xi \in \mathbb{R}^{n}$, and if for any $\beta \in \mathbb{N}_{0}^{n}$ with $|\beta| \leqslant q_{\psi}(s)$ there exists $\phi_{\beta} \in L^{1}\left(\mathbb{R}^{n}\right)$ such that for all $x, \xi \in \mathbb{R}^{n}$

$$
\left|\partial_{x}^{\beta} q_{2}(x, \xi)\right| \leqslant \phi_{\beta}(x) \psi(\xi)
$$


then $q_{2}$ has property $(\boldsymbol{P} .3)$ with

$$
\tilde{\gamma}(N)=c_{N} \sum_{|\beta \leqslant N|}\left\|\phi_{\beta}\right\|_{L^{1}\left(\mathbb{R}^{n}\right)}
$$

for all $0 \leqslant N \leqslant q_{\psi}(s)$, where the constants $c_{n}>0$ depend on $n$ and $N$ only.

We can associate a pseudo-differential operator $q_{2}(x, D)$, and if the functions $\hat{q}_{2}(\cdot, \eta)$ exist for all $\eta \in \mathbb{R}^{n}$ we get for $u \in C_{0}^{\infty}\left(\mathbb{R}^{n}\right)$

$$
\begin{aligned}
F_{\mathbb{R}^{n}}\left[q_{2}(x, D) u\right](\xi) & =(2 \pi)^{-n / 2} \int_{\mathbb{R}^{n}}(2 \pi)^{-n / 2} \int_{\mathbb{R}^{n}} e^{i x \eta} q_{2}(x, \eta) \hat{u}(\eta) d \eta d x \\
& =(2 \pi)^{-n / 2} \int_{\mathbb{R}^{n}} \hat{q_{2}}(\xi-\eta, \eta) \hat{u}(\eta) d \eta
\end{aligned}
$$

Lemma 6.9. Let $\psi: \mathbb{R}^{n} \rightarrow \mathbb{R}$ be a negative definite function, and let $q: \mathbb{R}^{n} \times \mathbb{R}^{n} \rightarrow$ $\mathbb{R}$ be decomposed into $q(x, \xi)=q_{1}(\xi)+q_{2}(x, \xi)$, where $q_{1}$ satisfies (P.2) and $q_{2}$ satisfies (P.3), $s \geqslant 0$. Then $q(x, D)$ can be extended to a continuous mapping from $H^{\psi, s+1}\left(\mathbb{R}^{n}\right)$ into $H^{\psi, s}\left(\mathbb{R}^{n}\right)$.

Now we want to discuss that the operator $-q(x, D)$ generates a Feller semigroup with using the Hille-Yosida-Ray Theorem, Theorem 2.65. Let $q(x, \xi)$ be a negative definite symbol and consider the operator $-q(x, D)$ on $C_{0}^{\infty}\left(\mathbb{R}^{n} ; \mathbb{R}\right)$. Compare [19], let $\psi: \mathbb{R}^{n} \rightarrow \mathbb{R}$ be a fixed continuous negative definite function satisfying for $c_{0}>0$, $r_{0}>0$ and all $|\xi| \geqslant 1$,

$$
\psi(\xi) \geqslant c_{0}|\xi|^{r_{0}}
$$

And let $t_{0}:=\left[\frac{n}{r_{0}}\right]+1$. Then we suppose that $q: \mathbb{R}^{n} \times \mathbb{R}^{n} \rightarrow \mathbb{C}$ is a continuous negative definite symbol have the form

$$
q(x, \xi)=q_{1}(\xi)+q_{2}(x, \xi)
$$

such that with $\psi$ as in (6.15) the following assumptions hold:

Assumption 6.10. 1. The function $q_{1}$ is assumed to be continuous negative definite and to satisfy with $\gamma_{0}>0$ and $\gamma_{1}, \gamma_{2} \geqslant 0$, for all $\xi \geqslant 1$

$$
\gamma_{0} \psi(\xi) \leqslant \boldsymbol{R e} q_{1}(\xi) \leqslant \gamma_{1} \psi(\xi)
$$


and for all $\xi \in \mathbb{R}^{n}$

$$
\left|\operatorname{Im} q_{1}(\xi)\right| \leqslant \gamma_{2} \operatorname{Re} q_{1}(\xi) .
$$

2. $m_{0}$. Set $m_{0}=t_{0}+n+2=\left[\frac{n}{r_{0}}\right]+n+3$, note $t_{0} \geqslant 1$. We assume that $x \mapsto q_{2}(x, \xi)$ belongs to $C^{m_{0}}\left(\mathbb{R}^{n}\right)$ for all $\xi \in \mathbb{R}^{n}$ and that we have the estimate

$$
\left|\partial_{x}^{\alpha} q_{2}(x, \xi)\right| \leqslant \psi_{\alpha}(x)(1+\psi(\xi))
$$

for all $\alpha \in \mathbb{N}_{0}^{\alpha},|\alpha| \leqslant m_{0}$, with functions $\psi_{\alpha} \in L^{1}\left(\mathbb{R}^{n}\right)$.

3. $m_{0}$. We require

$$
\sum_{\left|\alpha \leqslant m_{0}\right|}\left\|\psi_{\alpha}\right\|_{L^{1}} \leqslant \frac{1}{4} \gamma_{0}\left(\frac{1}{\kappa_{2}} \wedge \frac{1}{\tilde{c}_{n, m_{0}, \frac{t_{0}}{2}, \psi}}\right)
$$

where $\kappa_{2}$ and $\tilde{c}_{n, m_{0}, \frac{t_{0}}{2}, \psi}$ are referred from [19].

Theorem 6.11. Let Assumption 6.10 hold with $t_{0}=\left[\frac{n}{r_{0}}\right]+1$. Then $-q(x, D)$ extends to a generator of a Feller semigroup.

We shall need some $L^{2}$-bounds for certain classes of pseudo-differential operators. For this we give a presentation of the Calderón-Vaillancourt theorem following closely the presentation in [19]. Let $q: \mathbb{R}^{n} \times \mathbb{R}^{n} \rightarrow \mathbb{C}$ be a continuous function such that for all $\alpha, \beta \in \mathbb{N}_{0},|\alpha|,|\beta| \leqslant 3$, the partial derivatives $\partial_{x}^{\beta} \partial_{\xi}^{\alpha} q(x, \xi)$ exist, satisfy the estimates

$$
\left|\partial_{x}^{\beta} \partial_{\xi}^{\alpha} q(x, \xi)\right|<c_{\alpha, \beta} .
$$

We want to prove that $q(x, D)$ is bounded in $L^{2}\left(\mathbb{R}^{n}\right)$.

We give two auxiliary lemmas, the first one is

Lemma 6.12. Consider the function

$$
G(x):=\frac{1}{2} x^{2} e^{-x} \chi_{x \geqslant 0}(x)
$$

defined on $\mathbb{R}$ it is a fundamental solution of the differential operator $\left(1+\frac{d}{d x}\right)^{3}$, i.e.

$$
\left(1+\frac{d}{d x}\right)^{3} G=\varepsilon_{0}
$$

in the sense of distributions. 
Proof. (Compare [19, Lemma 2.5.2, p115])

By the definition of $E$, for all $\varphi \in C_{0}^{\infty}(\mathbb{R})$ and $\varepsilon>0$, we find

$$
\begin{aligned}
& \left\langle\left(1+\frac{d}{d x}\right)^{3} G, \varphi\right\rangle \\
& =\left\langle G,\left(1-\frac{d}{d x}\right)^{3} \varphi\right\rangle \\
& =\int_{0}^{\infty} \frac{1}{2} x^{2} e^{-x}\left(1-\frac{d}{d x}\right)^{3} \varphi(x) d x \\
& =\int_{\varepsilon}^{\infty}\left(1+\frac{d}{d x}\right)^{3}\left(\frac{1}{2} x^{2} e^{-x}\right) \varphi(x) d x+\int_{0}^{\varepsilon} \frac{1}{2} x^{2} e^{-x}\left(1-\frac{d}{d x}\right)^{3} \varphi(x) d x \\
& \quad+\varphi(\varepsilon) e^{-\varepsilon}-2 \varepsilon e^{-\varepsilon}\left\{\varphi(\varepsilon)+\varphi^{\prime}(\varepsilon)\right\}+\frac{1}{2} \varepsilon^{2} e^{-\varepsilon}\left\{\varphi(\varepsilon)+\varphi^{\prime}(\varepsilon)\right\} .
\end{aligned}
$$

For $x>0,\left(1+\frac{d}{d x}\right)^{3}\left(\frac{1}{2} x^{2} e^{-x}\right)=0$, and it follows as $\varepsilon \rightarrow 0$ that

$$
\left\langle\left(1+\frac{d}{d x}\right)^{3} G, \varphi\right\rangle=\varphi(0)
$$

which proves (6.23).

The second lemma is

Lemma 6.13. Let $(Z, \mathcal{A}, \mu)$ be a measure space. Let $A_{z}: L^{2}\left(\mathbb{R}^{n}\right) \rightarrow L^{2}\left(\mathbb{R}^{n}\right), z \in Z$, be a weakly measurable family of uniformly bounded operators, i.e. the mapping $z \mapsto A_{z}$ is weakly measurable and for all $z \in Z$ we have

$$
\left\|A_{z}\right\| \leqslant M_{0} .
$$

Here, $\|\cdot\|$ denotes the operator norm. Furthermore, we assume that $k: Z \times Z \rightarrow$ $\mathbb{R}_{+} \cap\{0\}$ is a non-negative function that give rise to a bounded integral operator $K_{\text {op }}$ on $L^{2}(Z ; \mu)$, i.e. $K_{o p}: L^{2}(Z ; \mu) \rightarrow L^{2}(Z ; \mu)$,

$$
K_{o p} u(z)=\int_{Z} k\left(z, z^{\prime}\right) u\left(z^{\prime}\right) d z^{\prime},
$$

with operator norm

$$
\left\|K_{o p}\right\|=M
$$

If the inequalities

$$
\left\|A_{z} A_{z^{\prime}}^{*}\right\| \leqslant k^{2}\left(z, z^{\prime}\right) \text { and }\left\|A_{z}^{*} A_{z^{\prime}}\right\| \leqslant k^{2}\left(z, z^{\prime}\right)
$$


hold, then the operator

$$
A:=\int_{Z} A_{z} d z
$$

is bounded in $L^{2}\left(\mathbb{R}^{n}\right)$ with norm $\|A\| \leqslant M$.

Proof. (Compare [19, Lemma 2.5.1, p113])

For $z_{1}, \ldots, z_{2 m} \in Z, m \in \mathbb{N}$, define

$$
T_{m}:=\left\|A_{z_{1}} A_{z_{2}}^{*} A_{z_{3}} \ldots A_{2 m}^{*}\right\| .
$$

Multiplying the two inequalities

$$
T_{m} \leqslant\left\|A_{z_{1}} A_{z_{2}}^{*}\right\| \cdot \ldots \cdot\left\|A_{z_{2 m-1}} A_{z_{2 m}}^{*}\right\|
$$

and

$$
T_{m} \leqslant\left\|A_{z_{1}}\right\|\left\|A_{z_{2}}^{*} A_{z_{3}}\right\| \cdot \ldots \cdot\left\|A_{z_{2 m-2}}^{*} A_{z_{2 m-1}}\right\|\left\|A_{z_{2 m}}^{*}\right\|
$$

we obtain

$$
T_{m}^{2} \leqslant\left\|A_{z_{1}}\right\|\left\|A_{z_{1}} A_{z_{2}}^{*}\right\|\left\|A_{z_{2}}^{*} A_{z_{3}}\right\| \cdot \ldots \cdot\left\|A_{z_{2 m-1}} A_{z_{2 m}}^{*}\right\|\left\|A_{z_{2 m}}^{*}\right\|
$$

Using (6.26) we find

$$
\begin{aligned}
& T_{m} \leqslant M_{0}^{1 / 2} k\left(z_{1}, z_{2}\right) k\left(k_{2}, k_{3}\right) \cdot \ldots \cdot k\left(z_{2 m-1}, z_{2 m}\right) M_{0}^{1 / 2} \\
& =M_{0} k\left(z_{1}, z_{2}\right) k\left(k_{2}, k_{3}\right) \cdot \ldots \cdot k\left(z_{2 m-1}, z_{2 m}\right) .
\end{aligned}
$$

Further, for a measurable set $N \subset Z$ such that $\mu(N)<\infty$ and for $u \in L^{2}\left(\mathbb{R}^{n}\right)$ such that $\|u\|_{0} \leqslant 1$ we get

$$
\begin{aligned}
& \left\|\left[\left(\int_{N} A_{z} d z\right)\left(\int_{N} A_{z} d z\right)^{*}\right]^{m} u\right\|_{0}^{1 / m} \\
& =\left\|\left(\int_{N} A_{z_{1}} d z_{1}\right)\left(\int_{N} A_{z_{2}} d z_{2}\right)^{*} \ldots\left(\int_{N} A_{z_{2 m-1}} d z_{2 m-1}\right)\left(\int_{N} A_{z_{2 m}} d z_{2 m}\right)^{*}\right\|_{0}^{1 / m} \\
& =\left\|\int_{N} \ldots \int_{N} A_{z_{1}} A_{z_{2}}^{*} \ldots A_{z_{2 m-1}} A_{z_{2 m}}^{*} d z_{1} \ldots d z_{2 m}\right\|_{0}^{1 / m} \\
& \leqslant\left(\int_{N} \ldots \int_{N}\left\|A_{z_{1}} A_{z_{2}}^{*} \ldots A_{z_{2 m-1}} A_{z_{2 m}}^{*}\right\| d z_{1} \ldots d z_{2 m}\right)^{1 / m} \\
& \leqslant\left|M_{0} \int_{N} \int_{N}\left\{\int_{Z} \ldots \int_{Z} k\left(z_{1}, z_{2}\right) \ldots k\left(z_{2 m-1}, z_{2 m}\right) d z_{2} \ldots d z_{2 m-1}\right\} d z_{1} d z_{2 m}\right|^{1 / m} \\
& =\left|M_{0} \int_{N} \int_{N} k^{2 m-1}\left(z_{1}, z_{2 m}\right) d z_{1} d z_{2 m}\right|^{1 / m},
\end{aligned}
$$


where $k^{(l)}$ denotes the $l$-times iterated kernel function. Hence we arrive at

$$
\begin{aligned}
& \left\|\left[\left(\int_{N} A_{z} d z\right)\left(\int_{N} A_{z} d z\right)^{*}\right]^{m} u\right\|_{0}^{1 / m} \\
& \leqslant\left(M_{0} \int_{Z} \int_{Z} \chi_{N}\left(z_{1}\right) k^{2 m-1}\left(z_{1}, z_{2 m}\right) \chi\left(z_{2 m}\right) d z_{2 m} d z_{1}\right)^{1 / m} \\
& \leqslant\left(M_{0} \mu(N) M^{2 m-1}\right)^{1 / m},
\end{aligned}
$$

which yields

$$
\left\|\left[\left(\int_{N} A_{z} d z\right)\left(\int_{N} A_{z} d z\right)^{*}\right]^{m} u\right\|_{0}^{1 / m} \leqslant\left(M_{0} \mu(N) M^{2 m-1}\right)^{1 / m}
$$

and as $m \rightarrow \infty$ it follows that

$$
\left\|\int_{N} A_{z} d z\right\|^{2} \leqslant M^{2}
$$

i.e.

$$
\left\|\int_{N} A_{z} d z\right\| \leqslant M
$$

and the lemma is proved.

Now we indicate the Calderón-Vaillancourt Theorem, see [5]:

Theorem 6.14. The pseudo-differential operator $q(x, D)$ satisfied $(6.21)$ which is defined on $\mathcal{S}\left(\mathbb{R}^{n}\right)$ by

$$
q(x, D) u(x)=(2 \pi)^{-1 / 2} \int_{\mathbb{R}} e^{i x \xi} q(x, \xi) \hat{u}(\xi) d \xi
$$

has a symbol satisfying (6.21) then it extends to a bounded operator from $L^{2}\left(\mathbb{R}^{n}\right)$ to itself.

Proof. (Compare [19, Theorem 2.5.3, p116])

We consider the function

$$
g(x, \xi):=\left(1+\frac{\partial}{\partial x}\right)^{3}\left(1+\frac{\partial}{\partial \xi}\right)^{3} p(x, \xi)
$$

Using the function $E$ from Lemma 6.12 we find

$$
q(x, \xi)=\int_{\mathbb{R}} \int_{\mathbb{R}} g(s, t) G(x-s) G(\xi-t) d s d t .
$$


Therefore on $\mathcal{S}(\mathbb{R})$ the operator $p(x, D)$ has the representation

$$
\begin{aligned}
& q(x, D) u(x)=(2 \pi)^{-1 / 2} \int_{\mathbb{R}} e^{i x \xi} \int_{\mathbb{R}} \int_{\mathbb{R}} g(s, t) G(x-s) G(\xi-t) d s d t \hat{u}(\xi) d \xi \\
& =(2 \pi)^{-1 / 2} \int_{\mathbb{R}} \int_{\mathbb{R}} g(s, t) \int_{\mathbb{R}} e^{i x \xi} G(x-s) G(\xi-t) \hat{u}(\xi) d \xi d t d s .
\end{aligned}
$$

Defining the operators

$$
\begin{aligned}
& A_{s t} u(x):=(2 \pi)^{-1 / 2} \int_{\mathbb{R}} e^{i x \xi} G(x-s) G(\xi-t) \hat{u}(\xi) d \xi \\
& =G(x-s)(2 \pi)^{-1 / 2} \int_{\mathbb{R}} e^{i x \xi} G(\xi-t) \hat{u}(\xi) d \xi
\end{aligned}
$$

we find

$$
q(x, D) u(x)=\int_{\mathbb{R}} \int_{\mathbb{R}} g(s, t) A_{s t} u(x) d s d t .
$$

We put $z=(s, t) \in \mathbb{R}^{2}$ and observe that $z=(s, t) \mapsto g(s, t)$ is measurable and bounded. It is sufficient to prove that the operator $A_{z}=A_{s t}$ satisfies the assumptions of Lemma 6.13 with $(Z, \mathcal{A}, \mu)=\left(\mathbb{R}^{2}, \mathcal{B}^{(2)}, \lambda^{(2)}\right)$. Since $\|G\|_{\infty} \leqslant 2 e^{-2}$ it follows that

$$
\begin{aligned}
\left\|A_{z} u\right\|_{0}^{2} & \leqslant\|G\|_{\infty}^{2}\left\|F^{-1}(G(\cdot-t) \hat{u}(\cdot))\right\|_{0}^{2} \\
& =\|G\|_{\infty}^{2}\|G(\cdot-t) \hat{u}(\cdot)\|_{0}^{2} \\
& \leqslant\|G\|_{\infty}^{4}\|\hat{u}\|_{0}^{2} \\
& =\|G\|_{\infty}^{4}\|u\|_{0}^{2},
\end{aligned}
$$

which gives

$$
\left\|A_{z}\right\| \leqslant 4 e^{-4}=: M_{0}
$$

Next we define the function

$$
k^{2}(s, t):=c_{0}^{2}(1+|s|)^{-3}(1+|t|)^{-3}
$$

where $c_{0}$ is a constant. Note that $k \in L^{1}\left(\mathbb{R}^{2}\right)$ and we put

$$
M:=c_{0}\left(\int_{\mathbb{R}}(1+|t|)^{-3 / 2} d t\right)^{2} .
$$

Take $(s, t),\left(s^{\prime}, t^{\prime}\right) \in \mathbb{R}^{2}$ and note that

$$
\begin{aligned}
H_{s t, s^{\prime} t^{\prime}}(x, y) & :=(2 \pi)^{-1 / 2} \int_{\mathbb{R}} e^{i(x-y) \cdot \xi} G(x-s) G(\xi-t) G\left(y-s^{\prime}\right) G\left(\xi-t^{\prime}\right) d \xi \\
& =G(x-s) G\left(y-s^{\prime}\right)(2 \pi)^{-1 / 2} \int_{\mathbb{R}} e^{i(x-y) \cdot \xi} G(\xi-t) G\left(\xi-t^{\prime}\right) d \xi
\end{aligned}
$$


is the kernel of the operator $A_{s t} A_{s^{\prime} t^{\prime}}^{*}$. With the change of variables

$$
\omega:=x-y, \eta:=\xi-t \text { and } \theta_{t}=t-t^{\prime} \geqslant 0
$$

we find for the integral in (6.35)

$$
\begin{aligned}
& (2 \pi)^{-1 / 2} e^{i \omega t} \int_{\mathbb{R}} e^{i \omega \eta} G(\eta) G\left(\eta+\theta_{t}\right) d \eta \\
& =(2 \pi)^{-1 / 2} \frac{1}{4} e^{i \omega t-\theta_{t}} \int_{0}^{\infty} e^{i \omega \eta} \eta^{2}\left(\eta+\theta_{t}\right)^{2} e^{-2 \eta} d \eta \\
& =(2 \pi)^{-1 / 2} \frac{1}{4} e^{i \omega t-\theta_{t}}\left(-i \frac{d}{d \omega}\right)^{2}\left(-i \frac{d}{d \omega}+\theta_{t}\right)^{2}\left(\frac{1}{2-i \omega}\right) .
\end{aligned}
$$

Analogously we may handle the case $\theta_{t}<0$ and we obtain a similar expression with $-\theta_{t}$. Thus it follows that

$$
\left|H_{s t, s^{\prime} t^{\prime}}(x, y)\right| \leqslant c e^{-\left|\theta_{t}\right| / 2}(1+|x-y|)^{-3} G(x-s) G\left(y-s^{\prime}\right),
$$

implying that

$$
\int_{\mathbb{R}} \int_{\mathbb{R}}\left|H_{s t, s^{\prime} t^{\prime}}(x, y)\right|^{2} d x d y \leqslant c e^{-\left|\theta_{t}\right| / 2} \int_{\mathbb{R}} \int_{\mathbb{R}}(1+|x-y|)^{-6} G^{2}(x-s) G^{2}\left(y-s^{\prime}\right) d x d y .
$$

The substitution $u+v=x-s$ and $u-v=y-s^{\prime}$ for $\theta_{s}:=s-s^{\prime} \geqslant 0$ yields

$$
\begin{aligned}
& \int_{\mathbb{R}} \int_{\mathbb{R}}(1+|x-y|)^{-6} G^{2}(x-s) G^{2}\left(y-s^{\prime}\right) d x d y \\
& =2 \int_{\mathbb{R}} \int_{\mathbb{R}}\left(1+\left|2 v+\theta_{s}\right|\right)^{-6} G^{2}(u+v) E^{2}(u-v) d u d v \\
& =\frac{1}{8} \int_{\mathbb{R}}\left(1+\left|2 v+\theta_{s}\right|\right)^{-6} \int_{u \geqslant|v|}\left(u^{2}-v^{2}\right) e^{-4 u} d u d v \\
& \leqslant c \int_{\mathbb{R}}\left(1+\left|2 v+\theta_{s}\right|\right)^{-6} \int_{u \geqslant|v|} e^{-u} d u d v \\
& =c \int_{\mathbb{R}}\left(1+\left|2 v+\theta_{s}\right|\right)^{-6} e^{|v|} d v .
\end{aligned}
$$

Using the Peetre-type estimate

$$
\left(1+\left|2 v+\theta_{s}\right|\right)^{-6} \leqslant \tilde{c}\left(1+\left|\theta_{s}\right|\right)^{-6}(1+|2 v|)^{6},
$$

it follows further from (6.36)

$$
\begin{aligned}
& \int_{\mathbb{R}} \int_{\mathbb{R}}(1+|x-y|)^{-6} G^{2}(x-s) G^{2}\left(y-s^{\prime}\right) d x d y \\
& \leqslant c^{\prime}\left(1+\left|\theta_{s}\right|\right)^{-6} \int_{\mathbb{R}}(1+|2 v|)^{6} e^{-|v|} d v \\
& \leqslant c^{\prime \prime}\left(1+\left|\theta_{s}\right|\right)^{-6} .
\end{aligned}
$$


Now we arrive at

$$
\begin{aligned}
& \left\|A_{s t} A_{s^{\prime} t^{\prime}}^{*}\right\|^{2} \\
& \leqslant \int_{\mathbb{R}} \int_{\mathbb{R}}\left|H_{s t, s^{\prime} t^{\prime}}(x, y)\right|^{2} d x d y \\
& \leqslant c\left(1+\left|s-s^{\prime}\right|\right)^{-6} e^{-2\left|t-t^{\prime}\right|} \\
& \leqslant c_{0}^{4}\left(1+\left|s-s^{\prime}\right|\right)^{-6}\left(1+\left|t+t^{\prime}\right|\right)^{-6} \\
& =k^{4}\left(s-s^{\prime}, t-t^{\prime}\right),
\end{aligned}
$$

which gives

$$
\left\|A_{s t} A_{s^{\prime} t^{\prime}}^{*}\right\|^{2} \leqslant k^{4}\left(s-s^{\prime}, t-t^{\prime}\right) .
$$

Since the kernel of $A_{s t}^{*} A_{s^{\prime} t^{\prime}}$ is given by

$$
(2 \pi)^{-1 / 2} G(\xi-t) G\left(\eta-t^{\prime}\right) \int_{\mathbb{R}} e^{-i x(\xi-\eta)} E(x-s) E\left(x-s^{\prime}\right) d x
$$

which is nothing but $\overline{H_{s t, s^{\prime} t^{\prime}}(\xi, \eta)}$, it follows further that

$$
\left\|A_{s t} A_{s^{\prime} t^{\prime}}^{*}\right\|^{2} \leqslant k^{4}\left(s-s^{\prime}, t-t^{\prime}\right)
$$

Thus the operators $\left(A_{z}\right)_{z=(s, t) \in \mathbb{R}^{2}}$ satisfy the assumptions of Lemma 6.13 with $M_{0}=$ $2 e^{-2}$ and $M$ given by (6.34). Hence we have proved the theorem for $n=1$. But the general case follows now by observing that we may handle each pair of variables $\left(x_{j}, \xi_{j}\right), 1 \leqslant j \leqslant n$, separately. 


\section{Chapter 7}

\section{A fraction-time, spatially}

\section{invariant, pseudo-differential}

\section{initial value problem}

We start by investigating the initial value problem

$$
{ }_{0} \mathcal{D}_{t}^{\alpha} u(x, t)+\psi\left(D_{x}\right) u(x, t)=0
$$

and

$$
\left.{ }_{0} \mathcal{D}_{t}^{\alpha-1} u(x, t)\right|_{t=0}=b(x)
$$

for $x \in \mathbb{R}, 0<t \leqslant T, T \leqslant \infty$, and suitable $b: \mathbb{R} \rightarrow \mathbb{R}$. Here $0<\alpha<1$ and ${ }_{0} \mathcal{D}_{t}^{\alpha}$ (and ${ }_{0} \mathcal{D}_{t}^{\alpha-1}$ ) denotes the Riemann-Liouville fractional derivative of the order $\alpha$ (and $\alpha-1$, respectively). Moreover $\psi: \mathbb{R} \rightarrow \mathbb{R}$ is a continuous negative definition function satisfying certain smoothness and growth condition. By $\psi\left(D_{x}\right)$ we denote the corresponding pseudo-differential operator which is at least defined for $u: \mathbb{R} \times[0, T] \rightarrow \mathbb{R}$, such that $u(\cdot, t) \in \mathcal{S}(\mathbb{R})$ for $t$ fixed. Recall that $[0, T]$ is the closed interval $0 \leqslant t \leqslant T$ for $T<\infty$ and for $T=\infty$ we mean the closed interval $[0, \infty)$. Following [34] we take in (7.1) and (7.2) a spatial Fourier transform

$$
\hat{u}(\xi, t):=(2 \pi)^{-1 / 2} \int_{\mathbb{R}} e^{-i x \xi} u(x, t) d x
$$


to get first

$$
\left(\psi\left(D_{x}\right) u(\cdot, t)\right)^{\wedge}(\xi, t)=\psi(\xi) \hat{u}(\xi, t)
$$

and eventually as transformed problem

$$
{ }_{0} \mathcal{D}_{t}^{\alpha} \hat{u}(\xi, t)+\psi(\xi) \hat{u}(\xi, t)=0
$$

and

$$
\left.{ }_{0} \mathcal{D}_{t}^{\alpha-1} \hat{u}(\xi, t)\right|_{t=0}=\hat{b}(\xi)
$$

Problem (7.5) and (7.6) we can consider as a parameter dependent fractional initial value problem. Under appropriate smoothness conditions we can solve this problem using a Laplace transform with respect to $t$. Denoting by $s \mapsto \hat{U}(\xi, s)$ the Laplace transform for the function $t \mapsto \hat{u}(\xi, t)$, i.e.

$$
\hat{U}(\xi, t):=\int_{0}^{\infty} e^{-s t} \hat{u}(\xi, t) d t
$$

we find for (7.5) and (7.6) by a formal calculation

$$
s^{\alpha} \hat{U}(\xi, s)+\psi(\xi) \hat{U}(\xi, s)=\hat{b}(\xi)
$$

or

$$
\hat{U}(\xi, s)=\frac{\hat{b}(\xi)}{s^{\alpha}+\psi(\xi)} .
$$

Using well-known formula for Laplace transforms, compare [34], we arrive formally at

$$
\hat{u}(\xi, t)=\hat{b}(\xi) t^{\alpha-1} E_{\alpha, \alpha}\left(-\psi(\xi) t^{\alpha}\right)
$$

Thus, we expect under suitable conditions

$$
\begin{aligned}
& u(x, t)=(2 \pi)^{-1 / 2} \int_{\mathbb{R}} e^{i x \xi} t^{\alpha-1} E_{\alpha, \alpha}\left(-\psi(\xi) t^{\alpha}\right) \hat{b}(\xi) d \xi \\
& =(2 \pi)^{-1 / 2} t^{\alpha-1}\left(F^{-1}\left(E_{\alpha, \alpha}\left(-\psi(\cdot) t^{\alpha}\right)\right) * b\right)(x, t)
\end{aligned}
$$

to be a solution of (7.1) and (7.2).

Note that for $\psi(\xi)=|\xi|^{2}$ this is exactly the result discussed in [34, p.140-p.141] for the equation

$$
{ }_{0} \mathcal{D}_{t}^{\alpha} u(x, t)-\frac{\partial^{2} u(x, t)}{\partial x^{2}}=0
$$


and

$$
\left.{ }_{0} \mathcal{D}_{t}^{\alpha-1} u(x, t)\right|_{t=0}=\varphi(x)
$$

As the first step towards our final result in this chapter we quote from [5, Proposition 5.1, p.309]:

Proposition 7.1. Let $0<\alpha<1, \psi: \mathbb{R} \rightarrow \mathbb{R}$ a continuous negative definite function and $b: \mathbb{R} \rightarrow \mathbb{R}$ a function with pointwisely definite Fourier transform, for example $b \in A(\mathbb{R})$, the Wiener algebra. The unique solution to (7.5) and (7.6) is given by formula (7.10).

In order to use this proposition to establish (7.11) we only need to assume that in the calculation leading from (7.5) and (7.6) to (7.11) we can reverse all steps as we need to guarantee that the steps leading from (7.1) and (7.2) are reversible.

First we note

Lemma 7.2. Suppose that $\psi: \mathbb{R} \rightarrow \mathbb{R}$ satisfies (5.2). If $\hat{b} \in L^{p}(\mathbb{R})$ for some $1 \leqslant p \leqslant \infty$, then $x \mapsto u(x, t)$, where $u$ is given by $(7.11)$ belongs to $C_{b}(\mathbb{R})$.

Proof. By Corollary 5.4 we know that $E_{\alpha, \alpha}\left(-\psi(\cdot) t^{\alpha}\right) \in L^{1}(\mathbb{R}) \cap L^{\infty}(\mathbb{R})$. Now Young's inequality, compare $[18$, p.31] yields the result.

Of course Lemma 7.2 does not give sufficient smoothness for (7.1) hold. In a later study we will consider Sobolev-type smoothness. Here we confine ourselves to the very smooth case, i.e. $b \in \mathcal{S}(\mathbb{R})$. We assume now that $\psi$ is at least of class $C^{2}$, i.e. we assume Lemma 5.6 to hold. In particular we will use $F^{-1}\left(E_{\alpha, \alpha}\left(-\psi(\cdot) t^{\alpha}\right)\right) \in L^{1}(\mathbb{R})$. In this case $u$ given by (7.11) is with respect to $x$ for $t>0$ fixed the convolution off an $L^{1}(\mathbb{R})$-function with a function in $\mathcal{S}(\mathbb{R})$ implying that $u(\cdot, t) \in C_{b}^{\infty}(\mathbb{R})$. Moreover, using Lemma 5.2 and Remark 5.3 it follows that

$$
\left(1+|x|^{2}\right)|u(x, t)| \leqslant c(t)
$$


Thus we conclude that $u(\cdot, t) \in C_{b}^{\infty}(\mathbb{R}) \cap L^{1}(\mathbb{R})$. For $b \in \mathcal{S}(\mathbb{R})$ we have $\hat{b} \in \mathcal{S}(\mathbb{R})$ and since $E_{\alpha, \alpha}\left(-\psi(\cdot) t^{\alpha}\right)$ is for $t>0$ fixed bounded, we deduce that $\left(1+|\cdot|{ }^{k / 2}\right) \hat{u}(\cdot, t) \in$ $L^{1}(\mathbb{R}) \cap L^{\infty}(\mathbb{R})$ for all $k \geqslant 0$ and $t>0$ fixed. This implies in particular that $\psi(\cdot) \hat{u}(\cdot, t) \in L^{1}(\mathbb{R})$ or $\psi\left(D_{x}\right) u(\cdot, t) \in C_{\infty}(\mathbb{R})$ for $t>0$ fixed. Eventually we obtain

Proposition 7.3. If $\psi: \mathbb{R} \rightarrow \mathbb{R}$ is a continuous negative definite function of class $C^{k}, k \geqslant 2$, satisfying (5.2) and if $b \in \mathcal{S}(\mathbb{R})$, then for $t>0$ the function (7.11) satisfies (7.1). Moreover, for $t \rightarrow 0$ it follows (7.2).

Proof. It remains to make a remark to (7.2). But the uniformly with respect to $\xi$ in (7.10) allows the passage to the limit, compare also in [26, Chapter 5 in particular $5.3]$.

Remark 7.4. The reader should put Proposition 7.3 in its right plane. It uses essentially well-known results on fractional initial value problems depending on a parameter. What we added is just a more detailed analysis of the parameter dependence due to its special structure, i.e. being at $\psi(\xi)$.

In a next step we want to discuss the problem

$$
{ }_{0} \mathcal{D}_{t}^{\alpha} u(x, t)+\psi\left(D_{x}\right) u(x, t)=g(x, t)
$$

and

$$
\left.{ }_{0} \mathcal{D}_{t}^{\alpha-1} u(x, t)\right|_{t=0}=b(x)
$$

Again we first apply a spatial Fourier transform to (7.15) and (7.16) to find

$$
{ }_{0} \mathcal{D}_{t}^{\alpha} \hat{u}(\xi, t)+\psi(\xi) \hat{u}(\xi, t)=\hat{g}(\xi, t)
$$

and

$$
\left.{ }_{0} \mathcal{D}_{t}^{\alpha-1} \hat{u}(\xi, t)\right|_{t=0}=\hat{b}(\xi)
$$


for $g: \mathbb{R} \times[0, \infty) \rightarrow \mathbb{R}$ and $b: \mathbb{R} \rightarrow \mathbb{R}$ being suitable functions. Solving the problem (7.17) and (7.18) using the Laplace transforms $\hat{U}(\xi, s)=\int_{0}^{\infty} e^{-s t} \hat{u}(\xi, t) d t$ and $\hat{G}(\xi, s)=\int_{0}^{\infty} e^{-s t} \hat{g}(\xi, t) d t$ we first arrive at

$$
s^{\alpha} \hat{U}(\xi, s)+\psi(\xi) \hat{U}(\xi, s)=\hat{G}(\xi, s)+\hat{b}(\xi)
$$

or

$$
\hat{U}(\xi, s)=\frac{\hat{G}(\xi, s)}{s^{\alpha}+\psi(\xi)}+\frac{\hat{b}(\xi)}{s^{\alpha}+\psi(\xi)} .
$$

Taking the inverse Laplace transform we find

$$
\hat{u}(\xi, t)=\hat{b}(\xi) t^{\alpha-1} E_{\alpha, \alpha}\left(-\psi(\xi) t^{\alpha}\right)+\int_{0}^{t}(t-\tau)^{\alpha-1} E_{\alpha, \alpha}\left(-\psi(\xi)(t-\tau)^{\alpha}\right) \hat{g}(\xi, t) d \tau
$$

Finally, using the convolution theorem we have the formal solution to (7.15) and (7.16) given by

$$
\begin{aligned}
u(x, t) & =(2 \pi)^{-1 / 2} t^{\alpha-1} \int_{\mathbb{R}} b(x-y)\left(F^{-1}\left(E_{\alpha, \alpha}\left(-\psi(\cdot) t^{\alpha}\right)\right)\right)(y) d y \\
& +(2 \pi)^{-1 / 2} t^{\alpha-1} \int_{\mathbb{R}} \int_{0}^{t} g(x-y, \tau)\left(F^{-1}\left(E_{\alpha, \alpha}\left(-\psi(\cdot)(t-\tau)^{\alpha}\right)\right)\right)(y) d \tau d y
\end{aligned}
$$

Once again, uniform (with respect to $t$ ) smoothness in $x$ will allow to reverse the arguments. As before $b \in \mathcal{S}(\mathbb{R})$ is sufficient. For $g$ matters are more delicate, however $g: \mathbb{R} \times[0, \infty) \rightarrow \mathbb{R}$ such that $g(\cdot, t)$ and $\hat{g}(\cdot, t)$ are in $\mathcal{S}(\mathbb{R})$ with semi-norm bounds uniform in $t$ and $g(x, \cdot)$ and $\hat{g}(\xi, \cdot)$ in $C_{b}^{1}(\mathbb{R}) \cap L^{1}(\mathbb{R})$ as well as $\frac{\partial}{\partial t} g(x, \cdot)$ and $\frac{\partial}{\partial t} \hat{g}(\xi, \cdot)$ in $C_{b}(\mathbb{R}) \cap L^{1}(\mathbb{R})$ with in all four cases norm bounds $\|h(x, \cdot)\|_{C_{b}}+\|h(x, \cdot)\|_{L^{1}}$ independent of $x$ will be sufficient.

Again we want to emphasize that we can rely on classical results, compare [34], [5] or [28] and we need only assume controls on the parameter $\psi(\xi)$.

In our main investigations we need to change our point of view and we will give a weaker notion of solution. We prepare this by giving

Definition 7.5. Let $0<\alpha<1, \psi: \mathbb{R} \rightarrow \mathbb{R}$ be a continuous negative definite function of class $C^{k}, k \geqslant 2$, satisfying the growth condition (5.2). Further let $b \in L^{1}(\mathbb{R}) \cap L^{\infty}(\mathbb{R})$ and $g \in L^{\infty}\left([0, T] ; L^{2}(\mathbb{R})\right)$ for some $T>0$. We call $u \in$ 
$L^{\infty}\left([0, T] ; L^{2}(\mathbb{R})\right)$ a mild solution to $(7.15)$ and $(7.16)$ if $u$ has representation $(7.22)$, i.e.

$$
\begin{aligned}
u(x, t) & =(2 \pi)^{-1 / 2} t^{\alpha-1} \int_{\mathbb{R}} b(x-y)\left(F^{-1}\left(E_{\alpha, \alpha}\left(-\psi(\cdot) t^{\alpha}\right)\right)\right)(y) d y \\
& +(2 \pi)^{-1 / 2} t^{\alpha-1} \int_{\mathbb{R}} \int_{0}^{t} g(x-y, \tau)\left(F^{-1}\left(E_{\alpha, \alpha}\left(-\psi(\cdot)(t-\tau)^{\alpha}\right)\right)\right)(y) d \tau d y
\end{aligned}
$$

Having Lemma 5.6 in mind we can easily deduce

Theorem 7.6. In the situation of Definition 7.5 problem (7.15) and (7.16) has for all $T>0$ a mild solution for $b \equiv 0$ and $g \in L^{\infty}\left([0, T] ; L^{2}(\mathbb{R})\right)$.

Proof. We only need to prove that $u$ given by $(7.23)$ with $g \in L^{\infty}\left([0, T] ; L^{2}(\mathbb{R})\right)$ belongs to $L^{\infty}\left([0, T] ; L^{2}(\mathbb{R})\right)$. Now, using Young's inequality we get

$$
\begin{aligned}
& \|u\|_{L^{\infty}\left([0, T] ; L^{2}(\mathbb{R})\right)} \\
& =\sup _{0 \leqslant t \leqslant T}(2 \pi)^{-1 / 2}\left\|\int_{\mathbb{R}} \int_{0}^{t}(t-\tau)^{\alpha-1} g(\cdot-y, \tau)\left(F^{-1}\left(E_{\alpha, \alpha}\left(-\psi(\cdot)(t-\tau)^{\alpha}\right)\right)\right)(y) d \tau d y\right\|_{L^{2}} \\
& \leqslant \sup _{0 \leqslant t \leqslant T}(2 \pi)^{-1 / 2} \int_{0}^{t}(t-\tau)^{\alpha-1}\left\|\int_{\mathbb{R}} g(\cdot-y, \tau)\left(F^{-1}\left(E_{\alpha, \alpha}\left(-\psi(\cdot)(t-\tau)^{\alpha}\right)\right)\right)(y) d y\right\|_{L^{2}} d \tau \\
& \leqslant(2 \pi)^{-1 / 2} \sup _{0 \leqslant t \leqslant T} \int_{0}^{t}(t-\tau)^{\alpha-1}\|g(\cdot, \tau)\|_{L^{2}}\left\|F^{-1}\left(E_{\alpha, \alpha}\left(-\psi(\cdot)(t-\tau)^{\alpha}\right)\right)\right\|_{L^{1}} d \tau \\
& \leqslant(2 \pi)^{-1 / 2}\|g\|_{L^{\infty}\left([0, T] ; L^{2}(\mathbb{R})\right)} \sup _{0 \leqslant t \leqslant T} \int_{0}^{t}(t-\tau)^{\alpha-1}\left\|F^{-1}\left(E_{\alpha, \alpha}\left(-\psi(\cdot)(t-\tau)^{\alpha}\right)\right)\right\|_{L^{1}} d \tau \\
& \leqslant(2 \pi)^{-1 / 2}\|g\|_{L^{\infty}\left([0, T] ; L^{2}(\mathbb{R})\right)}\left(\frac{\kappa_{1}}{\alpha(\gamma-1)} T^{\frac{\alpha}{\gamma}(\gamma-1)}+\frac{\kappa_{2}}{\alpha} T^{\alpha}\right),
\end{aligned}
$$

where we used in the last step Corollary 5.7.

Remark 7.7. We can not expect that for $b \neq 0$ we get a mild solution in $L^{\infty}\left([0, T] ; L^{2}(\mathbb{R})\right)$ due to the factor $t^{\alpha-1}$ and the decay of $\left\|F^{-1}\left(E_{\alpha, \alpha}\left(-\psi(\cdot) t^{\alpha}\right)\right)\right\|_{L^{1}}$ as $t$ tends to zero. However, for every $\varepsilon>0$ and $b \in L^{1}(\mathbb{R}) \cap L^{\infty}(\mathbb{R})$ we find that $u$ given by $(7.23)$ belongs to $L^{\infty}\left([\varepsilon, T] ; L^{2}(\mathbb{R})\right)$. 


\section{Chapter 8}

\section{A result for a fractional-time pseudo-differential initial value problem with space-dependent coefficients}

We now want to study the problem

$$
{ }_{0} \mathcal{D}_{t}^{\alpha} u(x, t)+\psi\left(D_{x}\right) u(x, t)+p\left(x, D_{x}\right) u(x, t)=g(x, t)
$$

and

$$
\left.{ }_{0} \mathcal{D}_{t}^{\alpha-1} u(x, t)\right|_{t=0}=0
$$

Here, $0<\alpha<1, \psi: \mathbb{R} \rightarrow \mathbb{R}$ is a continuous negative definite function of class $C^{k}$, $k \geqslant 2$, satisfying the growth condition (5.2), and

$$
p\left(x, D_{x}\right) v(x)=(2 \pi)^{-1 / 2} \int_{\mathbb{R}} e^{i x \xi} p(x, \xi) \hat{v}(\xi) d \xi
$$

is a pseudo-differential operator with symbol $p: \mathbb{R} \times \mathbb{R} \rightarrow \mathbb{R}$. We will discuss later on certain desirable properties of the symbol $p(x, \xi)$. In this paragraph, however, the only property needed is that $p\left(x, D_{x}\right)$ satisfies the estimate

$$
\left\|p\left(x, D_{x}\right) u(\cdot, t)\right\|_{L^{2}} \leqslant \mu\|u(\cdot, t)\|_{L^{2}}, u(\cdot, t) \in L^{2}(\mathbb{R})
$$


where $\mu$ is independent of $t$, and

$$
\|u(\cdot, t)\|_{L^{2}}^{2}=\int_{\mathbb{R}}|u(x, t)|^{2} d x .
$$

Using (7.23) and having in mind Definition 7.5 we give

Definition 8.1. Let $0<\alpha<1, \psi: \mathbb{R} \rightarrow \mathbb{R}$ as above and $g \in L^{\infty}\left([0, T] ; L^{2}(\mathbb{R})\right)$ for some $T>0$. We call $u \in L^{\infty}\left([0, T] ; L^{2}(\mathbb{R})\right)$ a mild solution to problem (8.1) and (8.2) if

$$
\begin{gathered}
u(x, t)=(2 \pi)^{-1 / 2} \int_{\mathbb{R}} \int_{0}^{t}(t-\tau)^{\alpha-1} g(x-y, \tau)\left(F^{-1}\left(E_{\alpha, \alpha}\left(-\psi(\cdot)(t-\tau)^{\alpha}\right)\right)\right)(y) d \tau d y \\
+(2 \pi)^{-1 / 2} \int_{\mathbb{R}} \int_{0}^{t}(t-\tau)^{\alpha-1}\left(p\left(x, D_{x}\right) u(x-y, \tau)\right) \\
\times\left(F^{-1}\left(E_{\alpha, \alpha}\left(-\psi(\cdot)(t-\tau)^{\alpha}\right)\right)\right)(y) d \tau d y
\end{gathered}
$$

holds.

Remark 8.2. The definition is justified, when looking at $p\left(x, D_{x}\right) u(x, t)$ as an additional right-hand side in (7.15).

Let us introduce the operator

$$
\begin{array}{rl}
L_{t} u(x, t):=(2 \pi)^{-1 / 2} \int_{\mathbb{R}} \int_{0}^{t}(t-\tau)^{\alpha-1} & g(x-y, \tau) \\
& \times\left(F^{-1}\left(E_{\alpha, \alpha}\left(-\psi(\cdot)(t-\tau)^{\alpha}\right)\right)\right)(y) d \tau d y \\
+(2 \pi)^{-1 / 2} \int_{\mathbb{R}} \int_{0}^{t}(t-\tau)^{\alpha-1}( & \left.p\left(x, D_{x}\right) u(x-y, \tau)\right) \\
& \times\left(F^{-1}\left(E_{\alpha, \alpha}\left(-\psi(\cdot)(t-\tau)^{\alpha}\right)\right)\right)(y) d \tau d y .
\end{array}
$$

It follows that $u \in L^{\infty}\left([0, T] ; L^{2}(\mathbb{R})\right)$ is a mild solution to (8.1) and (8.2) if and only if $u$ is a fixed point of $L_{t}$, i.e.

$$
L_{t} u=u
$$

We will now prove that under condition (8.4) the operator $L$ has a unique fixed point in $L^{\infty}\left([0, T] ; L^{2}(\mathbb{R})\right)$ provided $T>0$ is sufficiently small. 
Proposition 8.3. Suppose $0<\alpha<1, \psi: \mathbb{R} \rightarrow \mathbb{R}$ is a continuous negative defnition function of class $C^{k}, k \geqslant 2$, satisfying (5.2) and suppose that $p\left(x, D_{x}\right)$ satisfied (8.4). Further let $g \in L^{\infty}\left([0, T] ; L^{2}(\mathbb{R})\right)$. Then the operator $L_{t}$ satisfies on $L^{\infty}\left([0, T] ; L^{2}(\mathbb{R})\right)$ the Lipschitz estimate

$$
\left\|L_{t} u-L_{t} v\right\|_{L^{\infty}\left([0, T] ; L^{2}(\mathbb{R})\right)} \leqslant \lambda_{T}\|u-v\|_{L^{\infty}\left([0, T] ; L^{2}(\mathbb{R})\right)} .
$$

Proof. For $u, v \in L^{\infty}\left([0, T] ; L^{2}(\mathbb{R})\right)$ we have

$$
\begin{aligned}
& L_{t} u(\cdot, t)-L_{t} v(\cdot, t) \\
& \begin{aligned}
=(2 \pi)^{-1 / 2} \int_{\mathbb{R}} \int_{0}^{t}(t-\tau)^{\alpha-1}\left(F^{-1}\left(E_{\alpha, \alpha}\left(-\psi(\cdot)(t-\tau)^{\alpha}\right)\right)\right)(x-y) \\
\quad \times p\left(x, D_{x}\right)(u(y, \tau)-v(y, \tau)) d \tau d y
\end{aligned} \\
& \begin{aligned}
=(2 \pi)^{-1 / 2} \int_{0}^{t}\left((t-\tau)^{\alpha-1}\left(F^{-1}\left(E_{\alpha, \alpha}\left(-\psi(\cdot)(t-\tau)^{\alpha}\right)\right)\right)\right) \\
\quad *\left(p\left(x, D_{x}\right)(u(\cdot, \tau)-v(\cdot, \tau))\right)(x) d \tau .
\end{aligned}
\end{aligned}
$$

Using Young's inequality it follows that

$$
\begin{aligned}
& \left\|L_{t} u-L_{t} v\right\|_{L^{\infty}\left([0, T] ; L^{2}(\mathbb{R})\right)} \\
& \begin{array}{l}
\leqslant(2 \pi)^{-1 / 2} \sup _{0 \leqslant t \leqslant T} \int_{0}^{t}(t-\tau)^{\alpha-1}\left\|F^{-1}\left(E_{\alpha, \alpha}\left(-\psi(\cdot)(t-\tau)^{\alpha}\right)\right)\right\|_{L^{1}} \\
\quad \times\left\|p\left(x, D_{x}\right)(u(\cdot, \tau)-v(\cdot, \tau))\right\|_{L^{2}} d \tau \\
\leqslant(2 \pi)^{-1 / 2} \sup _{0 \leqslant t \leqslant T} \int_{0}^{t}(t-\tau)^{\alpha-1} h(t, \tau)\left\|p\left(x, D_{x}\right)(u(\cdot, \tau)-v(\cdot, \tau))\right\|_{L^{2}} d \tau
\end{array}
\end{aligned}
$$

where as in (5.13) we put

$$
h(t, \tau):=\left\|F^{-1}\left(E_{\alpha, \alpha}\left(-\psi(\cdot)(t-\tau)^{\alpha}\right)\right)\right\|_{L^{1}} .
$$

Using (8.4) we find

$$
\left\|p\left(x, D_{x}\right)(u(\cdot, \tau)-v(\cdot, \tau))\right\|_{L^{2}} \leqslant \mu\|u(\cdot, \tau)-v(\cdot, \tau)\|_{L^{2}}
$$

which implies by (5.15) that

$$
\begin{aligned}
& \left\|L_{t} u-L_{t} v\right\|_{L^{\infty}\left([0, T] ; L^{2}(\mathbb{R})\right)} \\
& \leqslant(2 \pi)^{-1 / 2} \mu\left(\sup _{0 \leqslant t \leqslant T} \int_{0}^{t}(t-\tau)^{\alpha-1} h(t, \tau) d \tau\right)\|u-v\|_{L^{\infty}\left([0, T] ; L^{2}(\mathbb{R})\right)} \\
& \leqslant(2 \pi)^{-1 / 2} \mu\left(\frac{\kappa_{1}}{\alpha(\gamma-1)} T^{\frac{\alpha}{\gamma}(\gamma-1)}+\frac{\kappa_{2}}{\alpha} T^{\alpha}\right)\|u-v\|_{L^{\infty}\left([0, T] ; L^{2}(\mathbb{R})\right)}
\end{aligned}
$$


and the proposition is proved.

Corollary 8.4. In the situation of Proposition 8.3, if $T>0$ is sufficiently small, then $L_{t}$ is a contraction on $L^{\infty}\left([0, T] ; L^{2}(\mathbb{R})\right)$, i.e. (8.9) holds with $\lambda_{T}<1$.

Proof. Since

$$
\lim _{T \rightarrow 0} T^{\frac{\alpha}{\gamma}(\gamma-1)}=\lim _{T \rightarrow 0} T^{\alpha}=0
$$

the result follows from (8.10).

It is obvious that Proposition 8.3 implies that for $g \equiv 0$ the only solution to (8.1) and (8.2) is the trivial one, i.e. $u \equiv 0$. For $g \neq 0$ we can use Banach's fixed point theorem to get a mild solution provided $T>0$ is sufficiently small and we have an invariant set containing $g$. The latter we establish in the following proposition.

Proposition 8.5. Suppose that the condition of Proposition 8.3 are satisfied. For $R>0$ consider

$$
B(g, R):=\left\{v \in L^{\infty}\left([0, T] ; L^{2}(\mathbb{R})\right) \mid\|v-g\|_{L^{\infty}\left([0, T] ; L^{2}(\mathbb{R})\right)} \leqslant R\right\}
$$

and assume

$$
\|g\|_{L^{\infty}\left([0, T] ; L^{2}(\mathbb{R})\right)} \leqslant \frac{R}{4} .
$$

Then there exist $T>0$ such that $L_{t}$ leaves $B(g, R)$ invariant, i.e. $u \in B(g, R)$ implies $L_{t} u \in B(g, R)$.

Proof. As before we may derive

$$
\begin{aligned}
& \left\|L_{t} u\right\|_{L^{\infty}\left([0, T] ; L^{2}(\mathbb{R})\right)} \\
& \leqslant(2 \pi)^{-1 / 2} \mu \int_{0}^{T}(t-\tau)^{\alpha-1} h(t, \tau) d \tau\|u\|_{L^{\infty}\left([0, T] ; L^{2}(\mathbb{R})\right)} \\
& \quad+(2 \pi)^{-1 / 2} \int_{0}^{T}(t-\tau)^{\alpha-1} h(t, \tau) d \tau\|g\|_{L^{\infty}\left([0, T] ; L^{2}(\mathbb{R})\right)}
\end{aligned}
$$


as well as

$$
\begin{aligned}
& \left\|L_{t} u-g\right\|_{L^{\infty}\left([0, T] ; L^{2}(\mathbb{R})\right)} \\
& \leqslant\left\|L_{t} u\right\|_{L^{\infty}\left([0, T] ; L^{2}(\mathbb{R})\right)}+\|g\|_{L^{\infty}\left([0, T] ; L^{2}(\mathbb{R})\right)} \\
& \leqslant(2 \pi)^{-1 / 2} \mu \int_{0}^{T}(t-\tau)^{\alpha-1} h(t, \tau) d \tau\|u\|_{L^{\infty}\left([0, T] ; L^{2}(\mathbb{R})\right)} \\
& \quad+(2 \pi)^{-1 / 2} \int_{0}^{T}(t-\tau)^{\alpha-1} h(t, \tau) d \tau\|g\|_{L^{\infty}\left([0, T] ; L^{2}(\mathbb{R})\right)}+\|g\|_{L^{\infty}\left([0, T] ; L^{2}(\mathbb{R})\right) .}
\end{aligned}
$$

Furthermore, for $u \in B(g, R)$ it follows that

$$
\|u\|_{L^{\infty}\left([0, T] ; L^{2}(\mathbb{R})\right)}-\|g\|_{L^{\infty}\left([0, T] ; L^{2}(\mathbb{R})\right)} \leqslant\|u-g\|_{L^{\infty}\left([0, T] ; L^{2}(\mathbb{R})\right)} \leqslant R
$$

or

$$
\|u\|_{L^{\infty}\left([0, T]_{i} L^{2}(\mathbb{R})\right)} \leqslant \frac{5 R}{4}
$$

Thus we arrive at

$$
\begin{aligned}
& \left\|L_{t} u-g\right\|_{L^{\infty}\left([0, T] ; L^{2}(\mathbb{R})\right)} \\
& \leqslant(2 \pi)^{-1 / 2} \mu \int_{0}^{T}(t-\tau)^{\alpha-1} h(t, \tau) d \tau \frac{5 R}{4} \\
& \quad+(2 \pi)^{-1 / 2} \int_{0}^{T}(t-\tau)^{\alpha-1} h(t, \tau) d \tau \frac{R}{4}+\frac{R}{4} \\
& \leqslant(2 \pi)^{-1 / 2} \max (1, \mu) \int_{0}^{T}(t-\tau)^{\alpha-1} h(t, \tau) d \tau \frac{3 R}{2}+\frac{R}{4}
\end{aligned}
$$

Again we can use (5.15) to find

$$
\left\|L_{t} u-g\right\|_{L^{\infty}\left([0, T] ; L^{2}(\mathbb{R})\right)} \leqslant(2 \pi)^{-1 / 2} \max (1, \mu)\left(\frac{\kappa_{1}}{\alpha(\gamma-1)} T^{\frac{\alpha}{\gamma}(\gamma-1)}+\frac{\kappa_{2}}{\alpha} T^{\alpha}\right) \cdot \frac{3 R}{2}+\frac{R}{4},
$$

and choosing $T>0$ such that $(2 \pi)^{-1 / 2} \max (1, \mu)\left(\frac{\kappa_{1}}{\alpha(\gamma-1)} T^{\frac{\alpha}{\gamma}(\gamma-1)}+\frac{\kappa_{2}}{\alpha} T^{\alpha}\right) \leqslant \frac{1}{2}$ we obtain the invariance of $B(g, R)$.

Combining Proposition 8.3 and Proposition 8.5 we eventually have

Theorem 8.6. Let $0<\alpha<0$ and $\psi: \mathbb{R} \rightarrow \mathbb{R}$ be a continuous negative definite function of class $C^{k}, k \geqslant 2$, satisfying (5.2). Suppose further that with $\kappa_{1}$ and $\kappa_{2}$ from (5.15) we have chosen $T>0$ such that

$$
2(2 \pi)^{-1 / 2} \max (1, \mu)\left(\frac{\kappa_{1}}{\alpha(\gamma-1)} T^{\frac{\alpha}{\gamma}(\gamma-1)}+\frac{\kappa_{2}}{\alpha} T^{\alpha}\right)<1
$$


Then $L_{t}$ is a contraction on $L^{\infty}\left([0, T] ; L^{2}(\mathbb{R})\right)$ and for $\|g\|_{L^{\infty}\left([0, T] ; L^{2}(\mathbb{R})\right)} \leqslant \frac{R}{4}$ the operator $L_{t}$ leaves $B(g, R) \subset L^{\infty}\left([0, T] ; L^{2}(\mathbb{R})\right)$ invariant. Consequently, $L_{t}$ has a unique fixed point which is a mild solution in the sense of Definition 8.1 to problem (8.1) and (8.2). 


\section{Chapter 9}

\section{Existence and Uniqueness for a}

\section{Class of Stochastic Fractional}

\section{Differential Equations}

In this chapter, let $(\Omega, \mathcal{F}, P)$ be a complete probability space with a filtration $\left\{\mathcal{F}_{t}\right\}_{t \geqslant 0}$ satisfying the usual conditions, i.e. it is right continuous and $\mathcal{F}_{0}$ contains all $P$-null sets. $B(t)$ is a standard Brownian motion defined on $(\Omega, \mathcal{F}, P)$. Now we want to modify the problem (8.1) and (8.2) as follows: Find a stochastic process $u=u(x, t ; w)$ such that

$$
{ }_{0} \mathcal{D}_{t}^{\alpha} u(x, t)+\psi\left(D_{x}\right) u(x, t)+p\left(x, D_{x}\right) u(x, t)-\int_{0}^{t} f(u(x, \theta)) d B(\theta)=g(x, t),
$$

with the initial data

$$
\left.{ }_{0} \mathcal{D}_{t}^{\alpha-1} u(x, t)\right|_{t=0}=0 .
$$

Here $\mathrm{u}(\mathrm{x}, \mathrm{t})$ is random variable. We work in the Banach space $L^{2}\left(\Omega \times \mathbb{R} ; L^{\infty}([0, T])\right)$ defined by

$$
L^{2}\left(\Omega \times \mathbb{R} ; L^{\infty}([0, T])\right):=\left\{u: \Omega \times \mathbb{R} \rightarrow L^{\infty}([0, T]) \mid\left(\mathbb{E} \int_{\mathbb{R}} \sup _{0 \leqslant t \leqslant T}|u(x, t)|^{2} d x\right)^{1 / 2}\right\}
$$

equipped with the norm

$$
\|u\|_{L^{2}\left(\Omega \times \mathbb{R} ; L^{\infty}([0, T])\right)}=\left(\mathbb{E} \int_{\mathbb{R}} \sup _{0 \leqslant t \leqslant T}|u(x, t)|^{2} d x\right)^{1 / 2} .
$$


Similar to Definition 8.1, we define the mild solution of problem (9.1) and (9.2) by

Definition 9.1. Let $0<\alpha<1, \psi: \mathbb{R} \rightarrow \mathbb{R}$ as above for some $T>0$. We call $u \in L^{2}\left(\Omega \times \mathbb{R} ; L^{\infty}([0, T])\right)$ a mild solution to problem (9.1) and (9.2) if

$$
\begin{aligned}
u(x, t)=(2 \pi)^{-1 / 2} \int_{\mathbb{R}} \int_{0}^{t}(t-\tau)^{\alpha-1} g(x-y, \tau)\left(F^{-1}\left(E_{\alpha, \alpha}\left(-\psi(\cdot)(t-\tau)^{\alpha}\right)\right)\right)(y) d \tau d y \\
+(2 \pi)^{-1 / 2} \int_{\mathbb{R}} \int_{0}^{t}(t-\tau)^{\alpha-1}\left(\int_{0}^{\tau} f(u(x-y, \theta)) d B(\theta)\right) \\
\quad \times\left(F^{-1}\left(E_{\alpha, \alpha}\left(-\psi(\cdot)(t-\tau)^{\alpha}\right)\right)\right)(y) d \tau d y \\
+(2 \pi)^{-1 / 2} \int_{\mathbb{R}} \int_{0}^{t}(t-\tau)^{\alpha-1}\left(p\left(x, D_{x}\right) u(x-y, \tau)\right) \\
\quad \times\left(F^{-1}\left(E_{\alpha, \alpha}\left(-\psi(\cdot)(t-\tau)^{\alpha}\right)\right)\right)(y) d \tau d y \quad \text { P-a.e. }
\end{aligned}
$$

holds.

Let us introduce the operator

$$
\begin{gathered}
L_{t} u(x, t)=(2 \pi)^{-1 / 2} \int_{\mathbb{R}} \int_{0}^{t}(t-\tau)^{\alpha-1} g(x-y, \tau)\left(F^{-1}\left(E_{\alpha, \alpha}\left(-\psi(\cdot)(t-\tau)^{\alpha}\right)\right)\right)(y) d \tau d y \\
+(2 \pi)^{-1 / 2} \int_{\mathbb{R}} \int_{0}^{t}(t-\tau)^{\alpha-1}\left(\int_{0}^{\tau} f(u(x-y, \theta)) d B(\theta)\right) \\
\quad \times\left(F^{-1}\left(E_{\alpha, \alpha}\left(-\psi(\cdot)(t-\tau)^{\alpha}\right)\right)\right)(y) d \tau d y \\
+(2 \pi)^{-1 / 2} \int_{\mathbb{R}} \int_{0}^{t}(t-\tau)^{\alpha-1}\left(p\left(x, D_{x}\right) u(x-y, \tau)\right) \\
\times\left(F^{-1}\left(E_{\alpha, \alpha}\left(-\psi(\cdot)(t-\tau)^{\alpha}\right)\right)\right)(y) d \tau d y .
\end{gathered}
$$

It follows that $u \in L^{2}\left(\Omega \times \mathbb{R} ; L^{\infty}([0, T])\right)$ is a mild solution to (9.1) and (9.2) if and only if $u$ is a fixed point of $L_{t}$, i.e.

$$
L_{t} u=u \quad \text { P-a.e.. }
$$


Theorem 9.2. Let $u, v \in L^{2}\left(\Omega \times \mathbb{R} ; L^{\infty}([0, T])\right)$ and let $f$ satisfy the Lipschitz conditions

$$
\|f(u)-f(v)\|_{L^{2}\left(\Omega \times \mathbb{R} ; L^{\infty}([0, T])\right)} \leqslant K_{1}\|u-v\|_{L^{2}\left(\Omega \times \mathbb{R} ; L^{\infty}([0, T])\right)}
$$

and linear growth condition

$$
\|f(u)\|_{L^{2}\left(\Omega \times \mathbb{R} ; L^{\infty}([0, T])\right)} \leqslant K_{2}\left(1+\|u\|_{L^{2}\left(\Omega \times \mathbb{R} ; L^{\infty}([0, T])\right)}\right)
$$

where $K_{1}$ and $K_{2}$ are two positive constants. For $R>0$ consider

$$
B(g, R):=\left\{v \in L^{2}\left(\Omega \times \mathbb{R} ; L^{\infty}([0, T])\right) \mid\|v-g\|_{L^{2}\left(\Omega \times \mathbb{R} ; L^{\infty}([0, T])\right)} \leqslant R\right\}
$$

and assume

$$
\|g\|_{L^{2}\left(\Omega \times \mathbb{R} ; L^{\infty}([0, T])\right)} \leqslant \frac{R}{4}
$$

Then there exists $T>0$ such that $L_{t}$ is a contraction on $L^{2}\left(\Omega \times \mathbb{R} ; L^{\infty}([0, T])\right)$ and $L_{t}$ leaves $B(g, R)$ invariant, i.e. $u \in B(g, R)$ implies $L_{t} u \in B(g, R)$. Consequently, $L_{t}$ has a unique fixed point which is a mild solution to the problem (9.1) and (9.2). 
Proof. For $u, v \in L^{2}\left(\Omega \times \mathbb{R} ; L^{\infty}([0, T])\right)$ we have

$$
\begin{aligned}
& \left\|L_{t} u-L_{t} v\right\|_{L^{2}\left(\Omega \times \mathbb{R} ; L^{\infty}([0, T])\right)} \\
& =\|(2 \pi)^{-1 / 2} \int_{\mathbb{R}} \int_{0}^{t}(t-\tau)^{\alpha-1} \int_{0}^{\tau}(f(u(x-y, \theta))-f(v(x-y, \theta))) d B(\theta) \\
& \times\left(F^{-1}\left(E_{\alpha, \alpha}\left(-\psi(\cdot)(t-\tau)^{\alpha}\right)\right)\right)(y) d \tau d y \\
& +(2 \pi)^{-1 / 2} \int_{\mathbb{R}} \int_{0}^{t}(t-\tau)^{\alpha-1}\left(p\left(x, D_{x}\right)(u(x-y, \tau)-v(x-y, \tau))\right) \\
& \times\left(F^{-1}\left(E_{\alpha, \alpha}\left(-\psi(\cdot)(t-\tau)^{\alpha}\right)\right)\right)(y) d \tau d y \|_{L^{2}\left(\Omega \times \mathbb{R} ; L^{\infty}([0, T])\right)} \\
& \leqslant(2 \pi)^{-1 / 2} \| \int_{\mathbb{R}} \int_{0}^{t}(t-\tau)^{\alpha-1} \int_{0}^{\tau}(f(u(x-y, \theta))-f(v(x-y, \theta))) d B(\theta) \\
& \times\left(F^{-1}\left(E_{\alpha, \alpha}\left(-\psi(\cdot)(t-\tau)^{\alpha}\right)\right)\right)(y) d \tau d y \|_{L^{2}\left(\Omega \times \mathbb{R} ; L^{\infty}([0, T])\right)} \\
& +(2 \pi)^{-1 / 2} \| \int_{\mathbb{R}} \int_{0}^{t}(t-\tau)^{\alpha-1}\left(p\left(x, D_{x}\right)(u(x-y, \tau)-v(x-y, \tau))\right) \\
& \times\left(F^{-1}\left(E_{\alpha, \alpha}\left(-\psi(\cdot)(t-\tau)^{\alpha}\right)\right)\right)(y) d \tau d y \|_{L^{2}\left(\Omega \times \mathbb{R} ; L^{\infty}([0, T])\right)} \\
& =(2 \pi)^{-1 / 2} \| \int_{0}^{t}(t-\tau)^{\alpha-1}\left(\int_{0}^{\tau}(f(u(\cdot, \theta))-f(v(\cdot, \theta))) d B(\theta)\right) \\
& *\left(F^{-1}\left(E_{\alpha, \alpha}\left(-\psi(\cdot)(t-\tau)^{\alpha}\right)\right)\right)(x) d \tau \|_{L^{2}\left(\Omega \times \mathbb{R}_{i} L^{\infty}([0, T])\right)} \\
& +(2 \pi)^{-1 / 2} \| \int_{0}^{t}(t-\tau)^{\alpha-1}\left(p\left(x, D_{x}\right)(u(\cdot, \tau)-v(\cdot, \tau))\right) \\
& *\left(F^{-1}\left(E_{\alpha, \alpha}\left(-\psi(\cdot)(t-\tau)^{\alpha}\right)\right)\right)(x) d \tau \|_{L^{2}\left(\Omega \times \mathbb{R} ; L^{\infty}([0, T])\right)} .
\end{aligned}
$$


In (9.12), we estimate the first term

$$
\begin{aligned}
& \| \int_{0}^{t}(t-\tau)^{\alpha-1}\left(\int_{0}^{\tau}(f(u(\cdot, \theta))-f(v(\cdot, \theta))) d B(\theta)\right) \\
& *\left(F^{-1}\left(E_{\alpha, \alpha}\left(-\psi(\cdot)(t-\tau)^{\alpha}\right)\right)\right)(x) d \tau d y \|_{L^{2}\left(\Omega \times \mathbb{R} ; L^{\infty}([0, T])\right)} \\
& =\left\{\mathbb { E } \int _ { \mathbb { R } } \operatorname { s u p } _ { 0 \leqslant t \leqslant T } \left(\int_{0}^{t}(t-\tau)^{\alpha-1}\left(\int_{0}^{\tau}(f(u(\cdot, \theta))-f(v(\cdot, \theta))) d B(\theta)\right)\right.\right. \\
& \left.\left.*\left(F^{-1}\left(E_{\alpha, \alpha}\left(-\psi(\cdot)(t-\tau)^{\alpha}\right)\right)\right)(x) d \tau\right)^{2} d x\right\}^{1 / 2} \\
& \leqslant\left\{\mathbb { E } \int _ { \mathbb { R } } \left(\sup _{0 \leqslant t \leqslant T} \int_{0}^{t}(t-\tau)^{\alpha-1}\left(\int_{0}^{\tau}(f(u(\cdot, \theta))-f(v(\cdot, \theta))) d B(\theta)\right)\right.\right. \\
& \left.\left.*\left(F^{-1}\left(E_{\alpha, \alpha}\left(-\psi(\cdot)(t-\tau)^{\alpha}\right)\right)\right)(x) d \tau\right)^{2} d x\right\}^{1 / 2} \\
& =\left\{\mathbb { E } \left[\left(\int _ { \mathbb { R } } \left(\sup _{0 \leqslant t \leqslant T} \int_{0}^{t}(t-\tau)^{\alpha-1}\left(\int_{0}^{\tau}(f(u(\cdot, \theta))-f(v(\cdot, \theta))) d B(\theta)\right)\right.\right.\right.\right. \\
& \left.\left.\left.\left.*\left(F^{-1}\left(E_{\alpha, \alpha}\left(-\psi(\cdot)(t-\tau)^{\alpha}\right)\right)\right)(x) d \tau\right)^{2} d x\right)^{1 / 2}\right]^{2}\right\}^{1 / 2} \\
& \leqslant\left\{\mathbb { E } \left(\sup _{0 \leqslant t \leqslant T} \int_{0}^{t}(t-\tau)^{\alpha-1}\left\|\int_{0}^{\tau}(f(u(\cdot, \theta))-f(v(\cdot, \theta))) d B(\theta)\right\|_{L^{2}}\right.\right. \\
& \left.\left.\times\left\|\left(F^{-1}\left(E_{\alpha, \alpha}\left(-\psi(\cdot)(t-\tau)^{\alpha}\right)\right)\right)(x) d \tau\right\|_{L^{1}}\right)^{2}\right\}^{1 / 2} \\
& =\left\{\mathbb{E}\left(\sup _{0 \leqslant t \leqslant T} \int_{0}^{t}(t-\tau)^{\alpha-1} h(t, \tau)\left\|\int_{0}^{\tau}(f(u(\cdot, \theta))-f(v(\cdot, \theta))) d B(\theta)\right\|_{L^{2}} d \tau\right)^{2}\right\}^{1 / 2}
\end{aligned}
$$

where as in (5.13) we put

$$
h(t, \tau):=\left\|F^{-1}\left(E_{\alpha, \alpha}\left(-\psi(\cdot)(t-\tau)^{\alpha}\right)\right)\right\|_{L^{1}} .
$$


Then, by using Burkholder-Davis-Gundy inequality, compare [29], we arrive at

$$
\begin{aligned}
& \| \int_{0}^{t}(t-\tau)^{\alpha-1}\left(\int_{0}^{\tau}(f(u(\cdot, \theta))-f(v(\cdot, \theta))) d B(\theta)\right) \\
& *\left(F^{-1}\left(E_{\alpha, \alpha}\left(-\psi(\cdot)(t-\tau)^{\alpha}\right)\right)\right)(x) d \tau d y \|_{L^{2}\left(\Omega \times \mathbb{R} ; L^{\infty}([0, T])\right)} \\
& \leqslant\left\{\mathbb { E } \left(\sup _{0 \leqslant t \leqslant T} \int_{0}^{t}(t-\tau)^{\alpha-1} h(t, \tau)\right.\right. \\
& \left.\left.\times\left\|\sup _{0 \leqslant \tau \leqslant T} \int_{0}^{\tau}(f(u(\cdot, \theta))-f(v(\cdot, \theta))) d B(\theta)\right\|_{L^{2}} d \tau\right)^{2}\right\}^{1 / 2} \\
& \leqslant\left(\frac{\kappa_{1}}{\alpha(\gamma-1)} T^{\alpha(\gamma-1) / \gamma}+\frac{\kappa_{2}}{\alpha} T^{\alpha}\right) \\
& \times\left\{\mathbb{E}\left(\left\|\sup _{0 \leqslant \tau \leqslant T} \int_{0}^{\tau}(f(u(\cdot, \theta))-f(v(\cdot, \theta))) d B(\theta)\right\|_{L^{2}}\right)^{2}\right\}^{1 / 2} \\
& =\left(\frac{\kappa_{1}}{\alpha(\gamma-1)} T^{\alpha(\gamma-1) / \gamma}+\frac{\kappa_{2}}{\alpha} T^{\alpha}\right) \\
& \times\left\{\mathbb{E} \int_{\mathbb{R}} \sup _{0 \leqslant \tau \leqslant T}\left(\int_{0}^{\tau}(f(u(\cdot, \theta))-f(v(\cdot, \theta))) d B(\theta)\right)^{2} d x\right\}^{1 / 2} \\
& \leqslant 4\left(\frac{\kappa_{1}}{\alpha(\gamma-1)} T^{\alpha(\gamma-1) / \gamma}+\frac{\kappa_{2}}{\alpha} T^{\alpha}\right)\left\{\int_{\mathbb{R}} \mathbb{E} \int_{0}^{\tau}(f(u(\cdot, \theta))-f(v(\cdot, \theta)))^{2} d \theta d x\right\}^{1 / 2} \\
& \leqslant 4 T^{1 / 2}\left(\frac{\kappa_{1}}{\alpha(\gamma-1)} T^{\alpha(\gamma-1) / \gamma}+\frac{\kappa_{2}}{\alpha} T^{\alpha}\right)\left\{\mathbb{E} \int_{\mathbb{R}} \sup _{0 \leqslant t \leqslant T}(f(u(\cdot, \cdot))-f(v(\cdot, \cdot)))^{2} d x\right\}^{1 / 2} \\
& \leqslant 4 T^{1 / 2} K_{1}\left(\frac{\kappa_{1}}{\alpha(\gamma-1)} T^{\alpha(\gamma-1) / \gamma}+\frac{\kappa_{2}}{\alpha} T^{\alpha}\right)\|u-v\|_{L^{2}\left(\Omega \times \mathbb{R} ; L^{\infty}([0, T])\right)}
\end{aligned}
$$


For the second term in (9.12), we have

$$
\begin{aligned}
& \| \int_{0}^{t}(t-\tau)^{\alpha-1}\left(p\left(x, D_{x}\right)(u(\cdot, \tau)-v(\cdot, \tau))\right) \\
& *\left(F^{-1}\left(E_{\alpha, \alpha}\left(-\psi(\cdot)(t-\tau)^{\alpha}\right)\right)\right)(x) d \tau \|_{L^{2}\left(\Omega \times \mathbb{R} ; L^{\infty}([0, T])\right)} \\
& =\left\{\mathbb { E } \int _ { \mathbb { R } } \operatorname { s u p } _ { 0 \leqslant t \leqslant T } \left(\int_{0}^{t}(t-\tau)^{\alpha-1}\left(p\left(x, D_{x}\right)(u(\cdot, \tau)-v(\cdot, \tau))\right)\right.\right. \\
& \left.\left.*\left(F^{-1}\left(E_{\alpha, \alpha}\left(-\psi(\cdot)(t-\tau)^{\alpha}\right)\right)\right)(x) d \tau\right)^{2} d x\right\}^{1 / 2} \\
& \leqslant\left\{\mathbb { E } \int _ { \mathbb { R } } \left(\sup _{0 \leqslant t \leqslant T} \int_{0}^{t}(t-\tau)^{\alpha-1}\left(p\left(x, D_{x}\right)(u(\cdot, \tau)-v(\cdot, \tau))\right)\right.\right. \\
& \leqslant\left\{\mathbb{E}\left(\sup _{0 \leqslant t \leqslant T} \int_{0}^{t}(t-\tau)^{\alpha-1} h(t, \tau)\left\|p\left(x, D_{x}\right)(u(\cdot, \tau)-v(\cdot, \tau))\right\|_{L^{2}} d \tau\right)^{2}\right\}^{1 / 2}
\end{aligned}
$$

where as in (5.13) we put

$$
h(t, \tau):=\left\|F^{-1}\left(E_{\alpha, \alpha}\left(-\psi(\cdot)(t-\tau)^{\alpha}\right)\right)\right\|_{L^{1}}
$$

Using (8.4) we find

$$
\left\|p\left(x, D_{x}\right)(u(\cdot, \tau)-v(\cdot, \tau))\right\|_{L^{2}} \leqslant \mu\|u(\cdot, \tau)-v(\cdot, \tau)\|_{L^{2}}
$$

which implies by (5.15) that

$$
\begin{aligned}
& \| \int_{0}^{t}(t-\tau)^{\alpha-1}\left(p\left(x, D_{x}\right)(u(\cdot, \tau)-v(\cdot, \tau))\right) \\
& *\left(F^{-1}\left(E_{\alpha, \alpha}\left(-\psi(\cdot)(t-\tau)^{\alpha}\right)\right)\right)(x) d \tau \|_{L^{2}\left(\Omega \times \mathbb{R} ; L^{\infty}([0, T])\right)} \\
& \leqslant\left\{\mathbb{E}\left(\sup _{0 \leqslant t \leqslant T} \int_{0}^{t}(t-\tau)^{\alpha-1} h(t, \tau) \sup _{0 \leqslant t \leqslant T} p\left(x, D_{x}\right)(u(\cdot, t)-v(\cdot, t)) \|_{L^{2}} d \tau\right)^{2}\right\}^{1 / 2} \\
& \leqslant \mu\left(\frac{\kappa_{1}}{\alpha(\gamma-1)} T^{\alpha(\gamma-1) / \gamma}+\frac{\kappa_{2}}{\alpha} T^{\alpha}\right)\|u-v\|_{L^{2}\left(\Omega \times \mathbb{R} ; L^{\infty}([0, T])\right)}
\end{aligned}
$$


Combining (9.14) and (9.16), we have

$\left\|L_{t} u-L_{t} v\right\|_{L^{2}\left(\Omega \times \mathbb{R} ; L^{\infty}([0, T])\right)}$

$\leqslant(2 \pi)^{-1 / 2}\left(\frac{\kappa_{1}}{\alpha(\gamma-1)} T^{\alpha(\gamma-1) / \gamma}+\frac{\kappa_{2}}{\alpha} T^{\alpha}\right)\left(4 K_{1} T^{1 / 2}+\mu\right)\|u-v\|_{\left.L^{2}\left(\Omega \times \mathbb{R} ; L^{\infty}(\mid 0, T]\right)\right)}$

In (9.17), let $\mathrm{T}$ be sufficiently small so that

$$
(2 \pi)^{-1 / 2}\left(\frac{\kappa_{1}}{\alpha(\gamma-1)} T^{\alpha(\gamma-1) / \gamma}+\frac{\kappa_{2}}{\alpha} T^{\alpha}\right)\left(4 K_{1} T^{1 / 2}+\mu\right)
$$

is less than or equal to 1 . Finally hence, $L_{t}$ is contractive.

Now, as before we may derive

$$
\begin{aligned}
& \left\|L_{t} u\right\|_{L^{2}\left(\Omega \times \mathbb{R} ; L^{\infty}([0, T])\right)} \\
& =\|(2 \pi)^{-1 / 2} \int_{\mathbb{R}} \int_{0}^{t}(t-\tau)^{\alpha-1} g(x-y, \tau) \\
& \quad \times\left(F^{-1}\left(E_{\alpha, \alpha}\left(-\psi(\cdot)(t-\tau)^{\alpha}\right)\right)\right)(y) d \tau d y \\
& +(2 \pi)^{-1 / 2} \int_{\mathbb{R}} \int_{0}^{t}(t-\tau)^{\alpha-1} \int_{0}^{\tau} f(u(x-y, \theta)) d B(\theta) \\
& \left.+(2 \pi)^{-1 / 2} \int_{\mathbb{R}} \int_{0}^{t}(t-\tau)^{\alpha-1} p\left(x, D_{x}\right) u(x-y, \tau)\right) \\
& \left.\left.\quad \times\left(F^{-1}\left(E_{\alpha, \alpha}\left(-\psi(\cdot)(t-\tau)^{\alpha}\right)\right)\right)(y) d \tau d y \|_{L^{2}\left(\Omega \times \mathbb{R} ; L^{\infty}([0, T])\right)}\left(-\psi(\cdot)(t-\tau)^{\alpha}\right)\right)\right)(y) d \tau d y \\
& \leqslant(2 \pi)^{-1 / 2} \| \int_{\mathbb{R}} \int_{0}^{t}(t-\tau)^{\alpha-1} g(x-y, \tau) \\
& \times(2 \pi)^{-1 / 2} \|_{\mathbb{R}} \int_{0}^{t}(t-\tau)^{\alpha-1} \int_{0}^{\tau} f(u(x-y, \theta)) d B(\theta) \\
& \left.\left.\times\left(F^{-1}\left(E_{\alpha, \alpha}\left(-\psi(\cdot)(t-\tau)^{\alpha}\right)\right)\right)(y) d \tau d y \|_{L^{2}\left(\Omega \times \mathbb{R} ; L^{\infty}([0, T])\right)}\left(-\psi(\cdot)(t-\tau)^{\alpha}\right)\right)\right)(y) d \tau d y \|_{L^{2}\left(\Omega \times \mathbb{R} ; L^{\infty}([0, T])\right)} \\
& +(2 \pi)^{-1 / 2} \| \int_{\mathbb{R}} \int_{0}^{t}(t-\tau)^{\alpha-1} p\left(x, D_{x}\right) u(x-y, \tau) \\
& \times\left(F^{-1}\left(E_{\alpha, \alpha}\left(-\psi(\cdot)(t-\tau)^{\alpha}\right)\right)\right)(y) d \tau d y \|_{L^{2}\left(\Omega \times \mathbb{R} ; L^{\infty}([0, T])\right)}
\end{aligned}
$$


In (9.18), we arrive at

$$
\begin{aligned}
& \left\|L_{t} u\right\|_{L^{2}\left(\Omega \times \mathbb{R} ; L^{\infty}([0, T])\right)} \\
& =(2 \pi)^{-1 / 2} \| \int_{0}^{t}(t-\tau)^{\alpha-1} g(\cdot, \tau) \\
& *\left(F^{-1}\left(E_{\alpha, \alpha}\left(-\psi(\cdot)(t-\tau)^{\alpha}\right)\right)\right)(x) d \tau d y \|_{L^{2}\left(\Omega \times \mathbb{R} ; L^{\infty}([0, T])\right)} \\
& +(2 \pi)^{-1 / 2} \| \int_{0}^{t}(t-\tau)^{\alpha-1}\left(\int_{0}^{\tau} f(u(\cdot, \theta)) d B(\theta)\right) \\
& *\left(F^{-1}\left(E_{\alpha, \alpha}\left(-\psi(\cdot)(t-\tau)^{\alpha}\right)\right)\right)(x) d \tau \|_{L^{2}\left(\Omega \times \mathbb{R} ; L^{\infty}([0, T])\right)} \\
& +(2 \pi)^{-1 / 2} \| \int_{0}^{t}(t-\tau)^{\alpha-1} p\left(x, D_{x}\right) u(\cdot, \tau) \\
& *\left(F^{-1}\left(E_{\alpha, \alpha}\left(-\psi(\cdot)(t-\tau)^{\alpha}\right)\right)\right)(x) d \tau \|_{L^{2}\left(\Omega \times \mathbb{R} ; L^{\infty}([0, T])\right)} \\
& \leqslant 4(2 \pi)^{-1 / 2} T^{1 / 2}\left(\frac{\kappa_{1}}{\alpha(\gamma-1)} T^{\alpha(\gamma-1) / \gamma}+\frac{\kappa_{2}}{\alpha} T^{\alpha}\right)\|f(u(\cdot, t))\|_{L^{2}\left(\Omega \times \mathbb{R} ; L^{\infty}([0, T])\right)} \\
& +\mu(2 \pi)^{-1 / 2}\left(\frac{\kappa_{1}}{\alpha(\gamma-1)} T^{\alpha(\gamma-1) / \gamma}+\frac{\kappa_{2}}{\alpha} T^{\alpha}\right)\|u\|_{L^{2}\left(\Omega \times \mathbb{R} ; L^{\infty}([0, T])\right)} \\
& +(2 \pi)^{-1 / 2}\left(\frac{\kappa_{1}}{\alpha(\gamma-1)} T^{\alpha(\gamma-1) / \gamma}+\frac{\kappa_{2}}{\alpha} T^{\alpha}\right)\|g\|_{L^{2}\left(\Omega \times \mathbb{R} ; L^{\infty}([0, T])\right)} .
\end{aligned}
$$

By using the linear growth condition (9.9), we have

$$
\begin{aligned}
& \left\|L_{t} u\right\|_{L^{2}\left(\Omega \times \mathbb{R} ; L^{\infty}([0, T])\right)} \\
& \leqslant 4(2 \pi)^{-1 / 2} T^{1 / 2}\left(\frac{\kappa_{1}}{\alpha(\gamma-1)} T^{\alpha(\gamma-1) / \gamma}+\frac{\kappa_{2}}{\alpha} T^{\alpha}\right) K_{2}\left(1+\|u\|_{L^{2}\left(\Omega \times \mathbb{R} ; L^{\infty}([0, T])\right)}\right) \\
& +\mu(2 \pi)^{-1 / 2}\left(\frac{\kappa_{1}}{\alpha(\gamma-1)} T^{\alpha(\gamma-1) / \gamma}+\frac{\kappa_{2}}{\alpha} T^{\alpha}\right)\|u\|_{L^{2}\left(\Omega \times \mathbb{R} ; L^{\infty}([0, T])\right)} \\
& +(2 \pi)^{-1 / 2}\left(\frac{\kappa_{1}}{\alpha(\gamma-1)} T^{\alpha(\gamma-1) / \gamma}+\frac{\kappa_{2}}{\alpha} T^{\alpha}\right)\|g\|_{L^{2}\left(\Omega \times \mathbb{R} ; L^{\infty}([0, T])\right)} \\
& =C_{1}+C_{1}\|u\|_{L^{2}\left(\Omega \times \mathbb{R} ; L^{\infty}([0, T])\right)}+C_{2}\|u\|_{L^{2}\left(\Omega \times \mathbb{R} ; L^{\infty}([0, T])\right)}+C_{3}\|g\|_{L^{2}\left(\Omega \times \mathbb{R} ; L^{\infty}([0, T])\right)},
\end{aligned}
$$

where

$$
\begin{gathered}
C_{1}:=4(2 \pi)^{-1 / 2} T^{1 / 2}\left(\frac{\kappa_{1}}{\alpha(\gamma-1)} T^{\alpha(\gamma-1) / \gamma}+\frac{\kappa_{2}}{\alpha} T^{\alpha}\right) K_{2} \\
C_{2}:=\mu(2 \pi)^{-1 / 2}\left(\frac{\kappa_{1}}{\alpha(\gamma-1)} T^{\alpha(\gamma-1) / \gamma}+\frac{\kappa_{2}}{\alpha} T^{\alpha}\right)
\end{gathered}
$$

and

$$
C_{3}:=(2 \pi)^{-1 / 2}\left(\frac{\kappa_{1}}{\alpha(\gamma-1)} T^{\alpha(\gamma-1) / \gamma}+\frac{\kappa_{2}}{\alpha} T^{\alpha}\right)
$$


Then we obtain

$$
\begin{aligned}
& \left\|L_{t} u-g\right\|_{L^{2}\left(\Omega \times \mathbb{R} ; L^{\infty}([0, T])\right)} \\
& \leqslant\left\|L_{t} u\right\|_{L^{2}\left(\Omega \times \mathbb{R}^{n} ; L^{\infty}([0, T])\right)}+\|g\|_{L^{2}\left(\Omega \times \mathbb{R} ; L^{\infty}([0, T])\right)} \\
& \leqslant C_{1}+C_{1}\|u\|_{L^{2}\left(\Omega \times \mathbb{R} ; L^{\infty}([0, T])\right)}+C_{2}\|u\|_{L^{2}\left(\Omega \times \mathbb{R} ; L^{\infty}([0, T])\right)} \\
& \quad+C_{3}\|g\|_{L^{2}\left(\Omega \times \mathbb{R} ; L^{\infty}([0, T])\right)}+\|g\|_{L^{2}\left(\Omega \times \mathbb{R} ; L^{\infty}([0, T])\right)} .
\end{aligned}
$$

Also for $u \in B(g, R)$ it follows

$$
\|u\|_{L^{2}\left(\Omega \times \mathbb{R}_{L^{\infty}}([0, T])\right)}-\|g\|_{L^{2}\left(\Omega \times \mathbb{R} ; L^{\infty}([0, T])\right)} \leqslant\|u-g\|_{L^{2}\left(\Omega \times \mathbb{R} ; L^{\infty}([0, T])\right)} \leqslant R
$$

or

$$
\|u\|_{L^{2}\left(\Omega \times \mathbb{R} ; L^{\infty}([0, T])\right)} \leqslant \frac{5 R}{4}
$$

Thus we arrive at

$$
\left\|L_{t} u-g\right\|_{L^{2}\left(\Omega \times \mathbb{R} ; L^{\infty}([0, T])\right)} \leqslant C_{1}+C_{1} \frac{5 R}{4}+C_{2} \frac{5 R}{4}+C_{3} \frac{R}{4}+\frac{R}{4} .
$$

Then we could make $C_{1}+C_{1} \frac{5 R}{4}+C_{2} \frac{5 R}{4}+C_{3} \frac{R}{4}+\frac{R}{4} \leqslant R$, i.e. $\frac{4 C_{1}}{3-5 C_{1}-5 C_{2}-C_{3}} \leqslant R$ and we find $\left\|L_{t} u-g\right\|_{L^{2}\left(\Omega \times \mathbb{R} ; L^{\infty}([0, T])\right)} \leqslant R$. 


\section{Notation}

\section{General Notation}

$\mathbb{N}$ natural number

$\mathbb{N}_{0}=\mathbb{N} \cup\{0\}$

$\mathbb{Q}$ rational number

$\mathbb{R}$ real number

$\mathbb{R}_{+}=\{x \in \mathbb{R} ; x \geqslant 0\}$

$\mathbb{R}^{n} \quad$ Euclidean vector space

$\mathbb{C}$ complex number

$a \wedge b=\min (a, b)$

$a \vee b=\max (a, b)$

$\operatorname{Re} z \quad$ real part of $z \in \mathbb{C}$

$\arg z \quad$ argument of $z \in \mathbb{C}$

$\operatorname{Im} z \quad$ imaginary part of $z$

\section{Function spaces and norms}

$C(G)$ continuous functions

$C_{0}(G)$ continuous functions with compact support

$C_{\infty}(G)$ continuous functions vanishing at infinity

$C^{k}(G) \quad k$-times continuously differentiable functions

$C_{0}^{k}(G)=C^{m}(G) \cap C_{0}(G)$ 
$C_{b}^{k}(G)=\left\{u \in C^{k}(G) ; \partial^{\alpha} u \in C_{b}(G),|\alpha| \leqslant k\right\}$

$C^{\infty}(G)=\bigcap_{m \in \mathbb{N}} C^{k}(G)$

$C_{0}^{\infty}(G)=\bigcap_{m \in \mathbb{N}} C_{0}^{k}(G)$

$H^{s}\left(\mathbb{R}^{n}\right) \quad$ classical Sobolev space of fractional order $s \in \mathbb{R}$

$H^{\psi, s}\left(\mathbb{R}^{n}\right)=\left\{u \in \mathcal{S}^{\prime}\left(\mathbb{R}^{n}\right) ;\|u\|_{\psi, s}<\infty\right\}$

$\mathcal{M}^{+}(\Omega)$ measures on $\Omega$

$\mathcal{M}_{b}^{+}(\Omega) \quad$ bounded measures on $\Omega$

$\mathcal{S}\left(\mathbb{R}^{n}\right) \quad$ Schwartz space of tempered functions

$\mathcal{S}^{\prime}\left(\mathbb{R}^{n}\right) \quad$ tempered distributions

\section{Functions, Function Transforms and operators}

$f \circ g \quad$ composition of functions

$f \otimes g \quad$ tensor product of functions or distributions

$f * g \quad$ convolution of functions or distributions

supp $f \quad$ support of a function or distribution

esssupp $f$ essential supremum of a function

$F[f], \hat{f} \quad$ Fourier transform

$F^{-1}[f]$ inverse Fourier transform

$\mathcal{L}(f) \quad$ Laplace transform of $f: \mathbb{R}_{+} \rightarrow \mathbb{C}$

$\mathcal{L}^{-1}(f) \quad$ inverse Laplace transform

$C N\left(\mathbb{R}^{n}\right) \quad$ continuous negative definite functions

$C P\left(\mathbb{R}^{n}\right) \quad$ continuous positive definite functions

$N\left(\mathbb{R}^{n}\right) \quad$ negative definite functions

$P\left(\mathbb{R}^{n}\right) \quad$ positive definite functions

$\Gamma$ Gamma function

$B$ Beta function

$E_{\alpha} \quad$ Mittag-Leffler function

$E_{\alpha, \beta} \quad$ two parameter Mittag-Leffler function 
$\psi \quad$ a continuous negative definite function

${ }_{0} I_{t}^{\alpha} \quad$ Riemann-Liouville fractional integral of the order $\alpha>0$

${ }_{0} I_{t}^{0} \quad$ Identity operator

$\mathcal{D}_{t}^{n} \quad$ standard derivative

${ }_{0} \mathcal{D}_{t}^{n} \quad$ Riemann-Liouville fractional derivative

${ }_{0}^{*} \mathcal{D}_{t}^{n} \quad$ Caputo fractional derivative 


\section{Bibliography}

[1] Barndorff-Nielsen, O., Processes of Normal Inverse Gaussian Type, Finance and Stochastics, 2 (1998), p.41-68.

[2] Berens, H., and Westphal, U., A Cauchy Problem for a Generalized Wave Equation, Acta Sci. Math. Szeged 29 (1968), p.93-106.

[3] Berg, Chr., and Forst, G., Potential Theory on Locally Compact Abelian Groups, Ergebnisse der Mathematik und ihrer Grenzgebiete, Band 87, Springer 1975.

[4] Butzer, P.L., and Berens, H., Semigroups of Operators and Approximation, Die Grundlehren der mathematischen Wissenschaften in Einzeldarstrllungen, Band 145, Springer 1967.

[5] Calderón, A.P., and Vaillancourt, R., On the Boundedness of Pseudodifferential Operators. J. Math. Soc. Japan 23 (1972), p.374-378.

[6] Erdélyi, A., Magnus, W., Oberhettinger, F., and Tricomi, F., Higher Transcendental Functions, vol. I-III, Krieger Pub., Melbourne, Florida, 1981.

[7] Gorenflo, R., and Mainardi, F., Fractional Calculus, Integral and Differential Equations of Fractional Order, Fractals and Fractional Calculus in Continuum Mechanics, CISM Courses and Lectures No.378, p.223-276, ed. A. Carpinteri, F. Mainardi, Springer 1997.

[8] Gorenflo, R., and Mainardi, F., Random Walk Models for Space-fractional Diffusion Processes. Fract. Cal. Appl. Anal. 1 (1998), 167-191. 
[9] Gorenflo, R., and Mainardi, F., Fractional Diffussion Processes: Probability Distribution and Continuous Time Random Walk. In : G. Rangarajan and M. Ding (eds.) "Processes with Long Range Correlations". Lecture Notes in Physis, vol.621, Springer Verlag, Berlin 2003, 148-166.

[10] Gorenflo, R., and Mainardi, F., Some Recent Advances in Theory and Simulation of Fractional Diffusion Processes. J. Comp. Appl. Math. 229 (2009),400415.

[11] Gorenflo, R., Vivoli, A., and Mainardi, F., Discrete and Continuous Random Walk Models for Space-time Fractional Diffusion. Nonlinear Dyn. 38 (2004), 101-106.

[12] Hardy, G.H., Littlewood, J.E., and Pólya, G., Inequalities, Cambridge University Press, 1934.

[13] Hilfer R., Threefold Introduction to Fractional Derivatives. In : R.Klages, G. Radons, and I.M. Sokolov (eds.) "Anomalous Transport: Foundations and Applications". Wiley-VCH, Weinheim 2008, 17-74.

[14] Hoh, W., A Symbolic Calculus for Pseudo-differential Operators Generating Feller Semigroups, Osaka J. Math 35 (1998), p.798-820.

[15] Hu, K., Jacob, N., and Yuan, C.G., On an equation being a fractional differential equation with respect to time and a pseudo-differential equation with respect to space related to Lvy-type processes. Fract. Cal. and Appl. Anal., Vol. 15, No. 1, (2012), 128-140.

[16] Jacob, N., A Class of Feller Semigroup Generated by Pseudo-differential Operators, Math. Z. 215 (1994), p.151-166.

[17] Jacob, N., Pseudo-differential Operators and Markov Processes, Mathematical Research, Vol.94, Akademie Verlag, 1996.

[18] Jacob, N., Pseudo-differential Operators and Markov Processes, vol. I: Fourier Analysis and Semigroups, Imperial College Press, 2001. 
[19] Jacob, N., Pseudo-differential Operators and Markov Processes, vol. II: Generators and Their Potential Theory, Imsperial College Press, 2002.

[20] Jacob, N., Pseudo-differential Operators and Markov Processes, vol. III: Markov Processes and Applications, Imperial College Press, 2005.

[21] Jacob, N., and Schilling R.L., An Analytic Proof of the Lévy-Khinchin Formula on $\mathbb{R}^{n}$, Publ. Math. Debrecen 53 (1998), p.69-89.

[22] Jacob, N., and Schilling, R.L., Fractional Derivatives, Non-symmetric and Time-dependent Dirichlet Forms and the Drift Form. Zeitschrift Anal. Anwendungen 19 (2000), p.801-830.

[23] Kato, T., Note on Fractional Powers of Linear Operators, Proc. Jap. Acad. 36 (1960), p.94-96.

[24] Kato, T., Fractional Powers of Dissipative Operators, J. Math. Soc. Japan 13(1961) No.3, p.246-274.

[25] Kilbas, A.A., Saigo M., and Saxena R.K., Generalized Mittag-Leffler Function and Generalized Fractional Calculus Operators, Integral Transform. Spec. Funct., 15(1) (2004),p.31-49.

[26] Kilbas, A.A., Srivastava H.M., and Trujillo J.J., Theory and Applications of Fractional Differential Equations, Elsevier, 2006.

[27] Komatsu, H., Fractional Powers of Operators, Pac. J. Math. 19 (1966) No.2, p.285-346.

[28] Mainardi, F., Fractional Calculus and Waves in Linear Viscoelasticity: An Introduction to Mathematical Models, Imperial College Press, 2010.

[29] Mao, X., Stochastic Differential Equations and Applications, Second Edition, Horwood Publishing Ltd. 2008.

[30] Meerschaert, Sochastic models for fractional calculus. De Gruyter Studies in Mathematics, vol.43, De Gruyter, Berlin 2012. 
[31] Meerschaert, M.M., Nane, E., and Vellaisamy, P., Fractional Cauchy Problems on Bounded Domains. Ann. Prob. 37 (2009), 979-1007.

[32] Metzler, R., and Klafter, J., The Random Walk's Guide to Anomalous Diffusion: A Fractional Dynamical Approach. Phys. Reports 339(2000), 1-77.

[33] Oldham, K.B., and Spanier, J., The fractional calculus. Mathematics in science and Engineering, vol. 111,Academic Press, New York, 1974.

[34] Podlubny, I., Fractional Differential Equations, Mathematics in Science and Engineering, Volume 198, Academic Press, 1998.

[35] Pollard, H., The completely monotonic character of the Mittag-Leffler function $E_{\alpha}(-x)$, Bull. Amer. Math. Soc. 54 (1948), 1115-1116.

[36] Pollard, H., The Representation of $\exp \left(-x^{\lambda}\right)$ as a Laplace Integral, Bull. Amer. Math. Soc. 52 (1948), p.908-910.

[37] Prabhakar, T.R., A Singular Integral Equation with a Generalized MittagLeffler Function in the Kernel, Yokohama Math. J., 19 (1971), 7-15.

[38] Samko, S.G., and Kilbas, A.A., and Marichev, O.I., Fractional Integrals and Derivatives - Theory and Applications, Gordon and Breach Science Publishers, 1993.

[39] Schilling, R.L., Zum Pfadverhalten von Markovschen Prozessen, die mit LévyProzessen Vergleichbar Sind. Dissertation Universität Erlangen-Nürnberg, Erlangen, 1994.

[40] Schilling, R.L., Negative Definite Functions of the Form $\psi(\xi)=f(|\xi|)$. Unpublished manuscript, Erlangen 1996.

[41] Schilling, R.L., Song, R.M., and Vondraček, Z., Bernstein Functions: Theory and Applications De Gruyter Study in Mathematics 37, 2010.

[42] Stein, E.M., Singular integrals and differentiability properties of functions, Princeton University Press, 1970. 
[43] Stein, E.M., and Weiss, G., Introduction to Fourier Analysis on Euclidean Spaces, Princeton Mathematical Series, Princeton University Press 1971.

[44] Weyl, H., Bemerkungen zum Begriff des Differentialquotienten gebrochener Ordnung. Vierteljahrschr. Naturforsch. Gesell. Zürich 62(1917), 296-302.

[45] Wiman, A., Über den Fundamentalsatz der Theorie der Funkntionen $E_{\alpha}(x)$, Acta Math. 29 (1905), p.191-201.

[46] Wong, R., and Zhao, Y.Q., Exponential Asymtotics of the Mittag-Leffler Function, Constructive Approximation 18, p.355-385.

[47] Yoshida, K., Functional Analysis, Die Grundlehren der mathematischen Wissenschaften in Einzeldarstellyngen, Vol. 123, springer 1965.

[48] Zhang, Y., Meerschaert, M.M., and Baeumer, B., Particle Tracking for TimeFractional Diffusion. Phys. Review E 78 (2008), 036705. 